\title{
Synchronization of Coupled and Periodically Forced Chemical Oscillators
}

Razan Snari

Follow this and additional works at: https://researchrepository.wvu.edu/etd

\section{Recommended Citation}

Snari, Razan, "Synchronization of Coupled and Periodically Forced Chemical Oscillators" (2015). Graduate Theses, Dissertations, and Problem Reports. 6678.

https://researchrepository.wvu.edu/etd/6678

This Dissertation is protected by copyright and/or related rights. It has been brought to you by the The Research Repository @ WVU with permission from the rights-holder(s). You are free to use this Dissertation in any way that is permitted by the copyright and related rights legislation that applies to your use. For other uses you must obtain permission from the rights-holder(s) directly, unless additional rights are indicated by a Creative Commons license in the record and/ or on the work itself. This Dissertation has been accepted for inclusion in WVU Graduate Theses, Dissertations, and Problem Reports collection by an authorized administrator of The Research Repository @ WVU.

For more information, please contact researchrepository@mail.wvu.edu. 


\title{
Synchronization of Coupled and Periodically Forced Chemical Oscillators
}

\author{
Razan Snari \\ Dissertation submitted \\ to the Eberly College of Arts and Sciences \\ at West Virginia University \\ in partial fulfillment of the requirements for the degree of \\ Doctor of Philosophy in \\ Chemistry
}
Kenneth Showalter, Ph.D., Chair
Charles Jaffé, Ph.D.
Terry Gullion, Ph.D.
Justin Legleiter, Ph.D.
Harvey Diamond, Ph.D.
Mark Tinsley, Ph.D.
Department of Chemistry

\author{
Morgantown, West Virginia \\ 2015
}

Keywords: Deep brain stimulation, Lyapunov exponent, phase-lag synchronization, phase response curves, photosensitive $\mathrm{BZ}$ reaction, synchronization

Copyright 2015 Razan Snari 


\section{ABSTRACT \\ Synchronization of Coupled and Periodically Forced Chemical Oscillators}

\section{Razan Snari}

Physiological rhythms are essential in all living organisms. Such rhythms are regulated through the interactions of many cells. Deviation of a biological system from its normal rhythms can lead to physiological maladies. The tremor and symptoms associated with Parkinson's disease are thought to emerge from abnormal synchrony of neuronal activity within the neural network of the brain. Deep brain stimulation is a therapeutic technique that can remove this pathological synchronization by the application of a periodic desynchronizing signal. Herein, we used the photosensitive Belousov-Zhabotinsky (BZ) chemical reaction to test the mechanism of deep brain stimulation. A collection of oscillators are initially synchronized using a regular light signal. Desynchronization is then attempted using an appropriately chosen desynchronizing signal based on information found in the phase response curve.

Coupled oscillators in various network topologies form the most common prototypical systems for studying networks of dynamical elements. In the present study, we couple discrete BZ photochemical oscillators in a network configuration. Different behaviors are observed on varying the coupling strength and the frequency heterogeneity, including incoherent oscillations to partial and full frequency entrainment. Phase clusters are organized symmetrically or non-symmetrically in phase-lag synchronization structures, a novel phase wave entrainment behavior in non-continuous media. The behavior is observed over a range of moderate coupling strengths and a broad frequency distribution of the oscillators. 


\section{Acknowledgments}

I would like foremost to thank Allah with my whole heart for making my education journey possible. My deepest gratitude is to my advisor, Dr. Kenneth Showalter, for providing the enthusiasm and work environment necessary to pursue my research studies. Thank you for your patience, faith, and continuous guidance. You are truly an inspiring Professor, mentor, and advisor. I also wish express my gratitude to the committee members, Dr. Charles Jaffé, Dr. Terry Gullion, Dr. Justin Legleiter, and Dr. Harvey Diamond, for taking the time to evaluate my dissertation, and Dr. Mark Tinsley, for his generosity, constructive criticism, and continuous guidance with experiments. I sincerely appreciate your responses to my queries and valuable suggestions for my dissertation. Thank you also to the research group members, Dr. Simbarashe Nkomo, Dr. Hua Ke, Dr. Calistus Ngonghala, Jan Toz, Desmond Yengi, Darrell Collison, Sadegh Faramarzi Ganjabad, and Tianran Chen, for your help and advice. I am blessed to have worked with all of you during my graduate education. I would like to express my deepest thanks to my family and friends for their encouragement and support throughout my education. The support and love of my husband, Essam Koshak, and my five children, Anas, Leen, Bassel, Ammar, and Lamaar, surrounded me during my whole international learning journey. I am particularly grateful to my mother, Samya Alsayed, for your continuous prayers and love. I am also very fortunate to have a special father, Mohammed Snari, who provided me with ultimate love and support. Although you passed away prior to my final dissertation, your encouraging words remain in my mind in every step of my life, making me the person I am now. 


\section{Table of Contents}

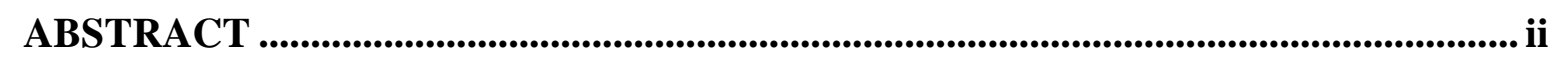

Acknowledgments.........................................................................................................................................ii

Table of Contents............................................................................................................................. iv

List of Figures ............................................................................................................................................. vii

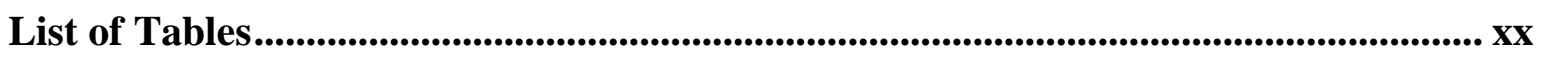

1. Synchronization ...................................................................................................................... 1

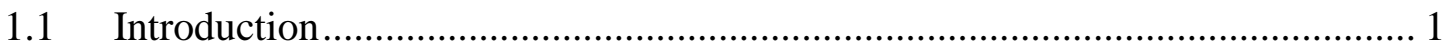

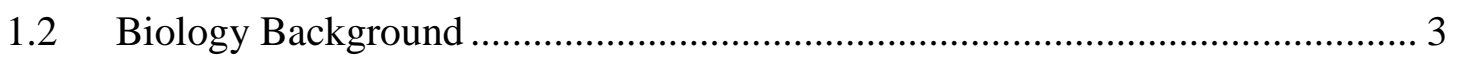

1.3 Chemistry Background: The BZ Reaction...................................................... 6

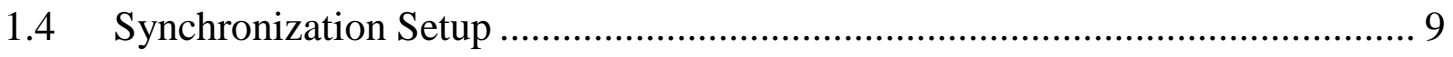

1.4.1 Synchronization via Mutual Coupling .......................................................... 9

1.4.2 Synchronization via External Forcing ...................................................... 15

2. Photosensitive BZ Chemical Oscillators......................................................................... 29

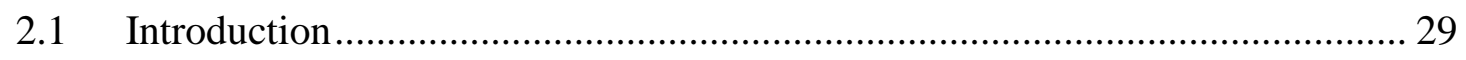

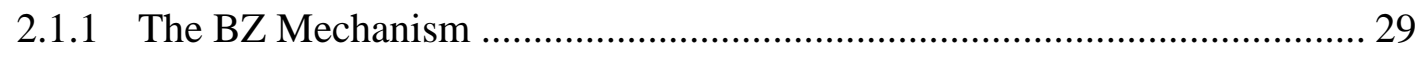

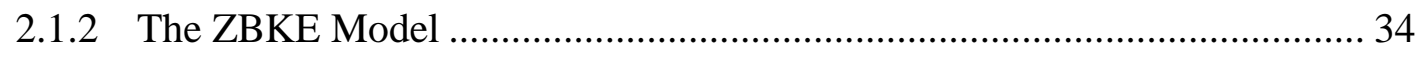

2.1.3 The Modified ZBKE Model for the Photosensitive BZ System................... 38

2.2 Phase Response Curve with Positive and Negative Perturbations..................... 40

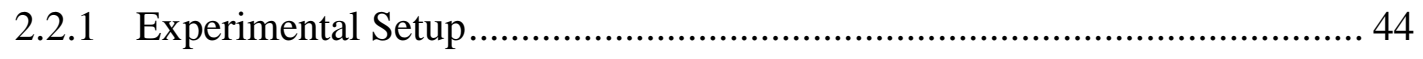

2.2.1.1 Preparation of Ruthenium (II) Catalyst Particles.................................... 45 
2.2.1.2 Preparation of Catalyst-Free BZ Solution ............................................ 45

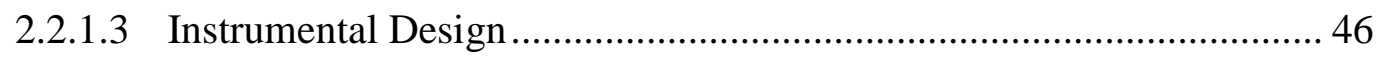

2.2.2 PRC Experimental Technique and Procedures ........................................... 46

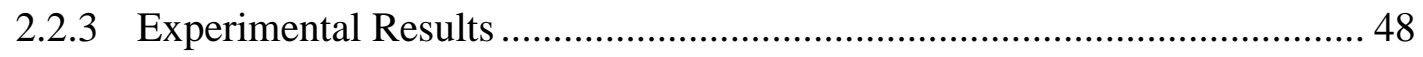

\section{Synchronization and Desynchronization in Populations of Uncoupled Chemical}

Oscillators with Periodic Forcing ........................................................................................ 61

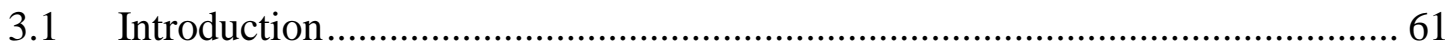

3.2 Synchronization and Desynchronization Measurements .................................... 63

3.2.1 Lyapunov Exponent and the Phase Response Curve .................................... 63

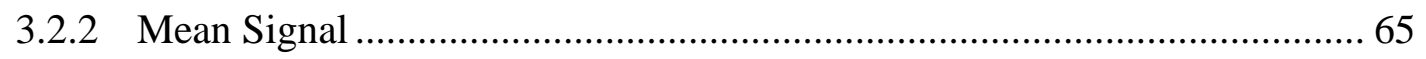

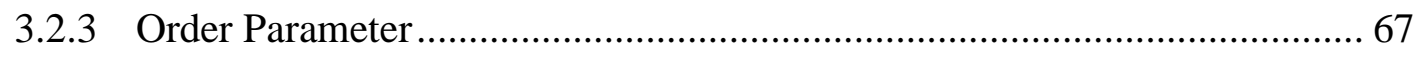

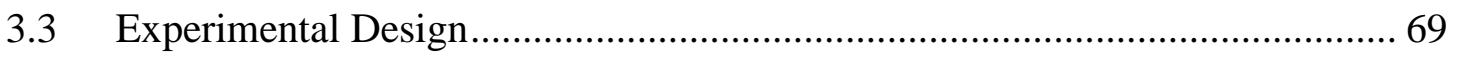

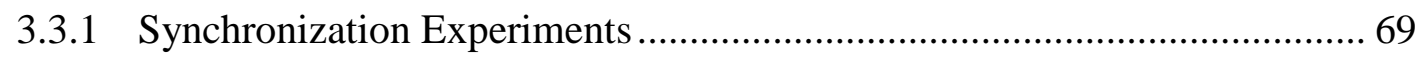

3.3.2 Desynchronization Experiments .............................................................. 72

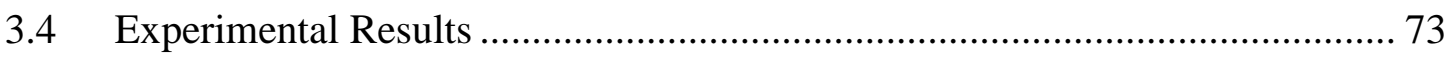

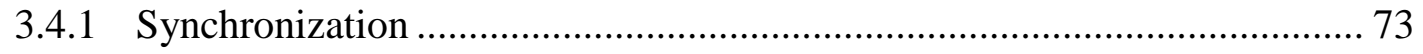

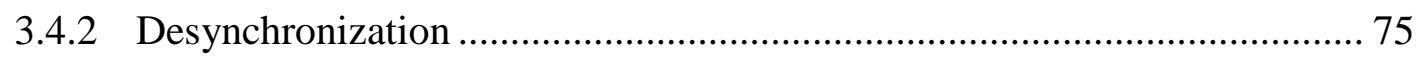

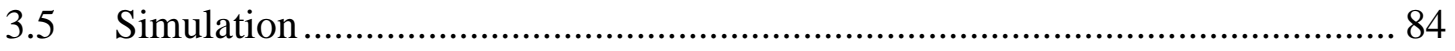

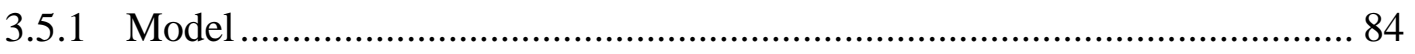

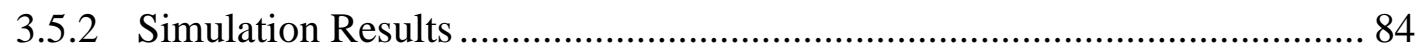

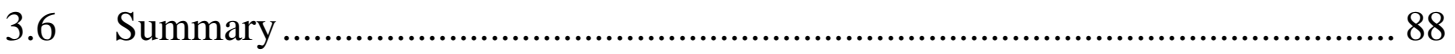

4. Desynchronization in Populations of Uncoupled Chemical Oscillators with

Colored Noise by Periodic Forcing .............................................................................. 96 


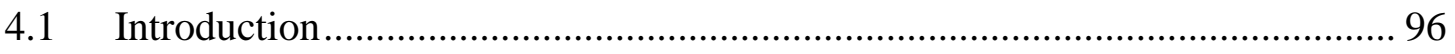

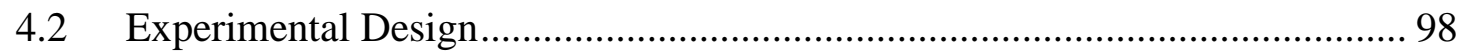

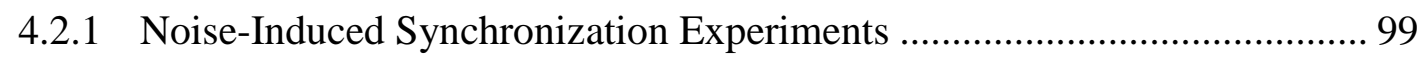

4.2.2 Desynchronization Experiments ....................................................... 101

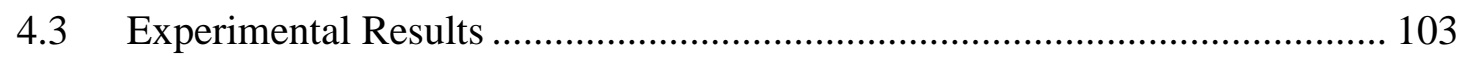

4.3.1 Signal in the Presence of Noise ..................................................... 103

4.3.2 Signal in the Presence of Noise and Negative Perturbations .................... 106

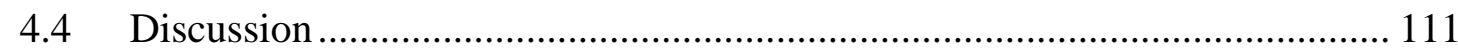

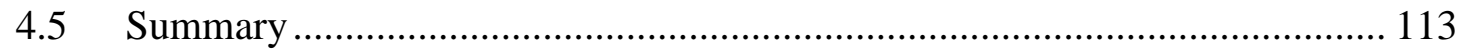

5. Phase-Lag Synchronization in a Chemical Oscillator Network......................... 123

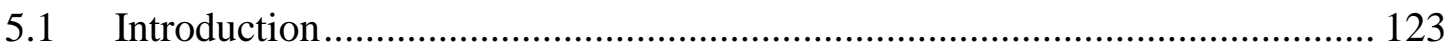

5.2 Network Experimental Design .......................................................... 125

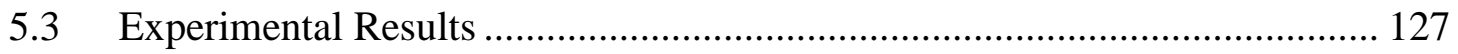

5.3.1 Symmetry Cluster Phase-Lag Synchronization .................................. 127

5.3.2 Partial Symmetry Cluster Phase-Lag Synchronization............................ 129

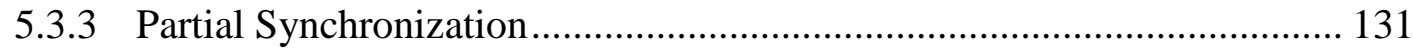

5.3.4 Independent Phase Oscillations ......................................................... 132

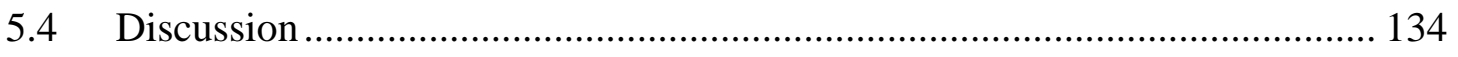

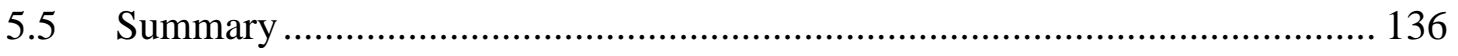




\section{List of Figures}

FIG. 1.1: Individual time series showing synchronization in the chemical BelousovZhabotinsky (BZ) system. BZ reaction mixture composition: malonic acid, [MA] = $0.08 \mathrm{M}$; sodium bromide, $[\mathrm{NaBr}]=0.02 \mathrm{M}$; sulfuric acid, $\left[\mathrm{H}_{2} \mathrm{SO}_{4}\right]=0.78 \mathrm{M}$; sodium bromate, $\left[\mathrm{NaBrO}_{3}\right]=0.48 \mathrm{M}$. System of 20 micro-oscillators loaded with

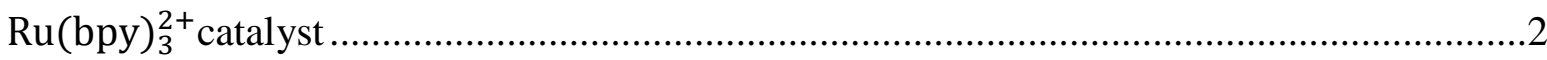

FIG. 1.2: Basal ganglia structures. Illustrated image is from ref. [15] .......................................4

FIG. 1.3: Deep brain stimulation. a) A cross section of the brain shows the electrode placement in the subthalamic nucleus for deep brain stimulation for Parkinson's disease. b) Illustration of the stereotactic device. Illustrated images are from ref. [21]

FIG. 1.4: A limit cycle curve of periodic oscillations in the phase plane and the corresponding time series plot. Points with the same numbers are of the same phase and are repeated for each period or cycle. Schematic diagram is from ref. [2]

FIG. 1.5: Relaxation oscillators. a) A relaxation Belousov-Zhabotinsky (BZ) oscillation from a model simulation with two variables, $\mathrm{x}$ and $\mathrm{z}$, where $\mathrm{x}$ represents the fast variable, bromous acid, and $\mathrm{z}$ represents the slow variable, the catalyst. b) A relaxation BZ oscillation shown in the catalyst concentration in a BZ reaction experiment. c) Rapid neural firing generates a spike each time the cell potential slowly reaches $-53 \mathrm{mV}$, and the cell then discharges and is reset to $-70 \mathrm{mV}$. Schematic diagram is from ref. [34]

FIG. 1.6: Two main types of synchronization. a) In-phase synchronization of two oscillators.

b) Antiphase synchronization of two oscillators 
FIG. 1.7: Kuramoto model plot describing the order parameter $r$ to measure oscillator synchronization as a function of the coupling strength, $\mathrm{K}$. The transition to synchronization occurs at the critical threshold of the coupling strength $\mathrm{K}_{\mathrm{c}}$

FIG. 1.8: Natural period distributions. a) A narrow distribution of natural periods. b) A wide distribution of natural periods

FIG. 1.9: Network connections. a) An all-to-all network, where each node is connected to all other nodes in the network. b) A bidirectional network, where pairs of oscillators influence each other equally. c) A unidirectional network, where the interaction is in one direction.

FIG. 1.10: Perturbation influence on oscillations. The external weak perturbation changes the phase point (filled circle) to a new position in the perturbed state (dashed circles). The initial phase of the oscillator is $\phi_{0}$. Schematic diagram is from ref. [2]

FIG. 1.11: The relationship between the forcing amplitude and frequency detuning, i.e., how much the external forcing frequency $\omega$ differs from the oscillator frequency $\omega_{0}$. The gray area reflects the Arnold tongue for the synchronization region. Schematic diagram adapted from ref. [2]

FIG. 2.1: Simulation of the Belousov-Zhabotinsky (BZ) reaction with the ZBKE model. a) The oscillation in bromous acid, X (blue), and the catalyst, Z (red). b) A plot of X vs. Z showing the limit cycle of the $\mathrm{BZ}$ reaction.

FIG. 2.2: Zhabotinsky, Buchholtz, Kiyatkin, and Epstein (ZBKE) and photo-ZBKE simulations. a) Time series showing oscillation in the catalyst, Z, with a longer oscillatory period, $3.93 \times 10^{5}$, in the typical Belousov-Zhabotinsky (BZ) system. b) 
Time series showing oscillation in the catalyst, Z, with a shorter oscillatory period, $3.43 \times 10^{5}$, in the photosensitive BZ system

FIG. 2.3: Time-phase conversion. a) A time series showing the successive periods $\mathrm{T}_{0}$, each starting at the spiking event $t_{\text {peak }}$ b) The corresponding phase representation of the time series where each period is of size $2 \pi$........

FIG. 2.4: Phase and a phase shift illustration in a phase response curve experiment. Periodic perturbation, $t_{s}$ (green lines), applied at different phase of the cycle $\theta \in[0,2 \pi)$, where

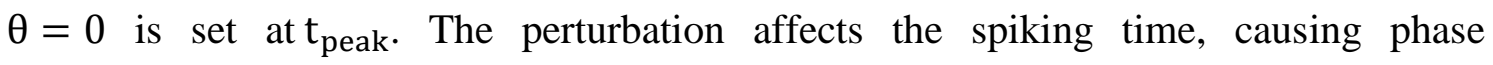
advancement. This example is from the Belousov-Zhabotinsky excitatory experiment with a negative perturbation. The natural period was $\mathrm{T}_{0}$ and the period shortens to $\mathrm{T}_{1}$ after the perturbation.

FIG. 2.5: Phase response curve experiment. The interval of successive perturbations (green lines) requires inclusion of at least one unperturbed cycle to define a phase shift according to a prior unperturbed period.

FIG. 2.6: Simulation of dark perturbation (blue plot) and light perturbation (red plot). Plotted data is from ref. [21]

FIG. 2.7: Experimental setup. The light is supplied from the projector only, and is filtered and reflected by a beam splitter onto the ruthenium-loaded particles. Images are recorded in real time by the camera and processed via a computer.

FIG. 2.8: Arrangement of catalyst particles in a catalyst-free Belousov-Zhabotinsky solution to maintain a discrete uncoupled system 
FIG. 2.9: An averaged and smoothed PRC of several oscillators. a) A phase response curve (PRC) of 19 individual oscillators with negative perturbations. b) The smoothed PRC from 19 beads in the PRC experiment

FIG. 2.10: PRC from negative and positive perturbations. a) Experimental data from negative perturbation (blue plot), I $=-1.67 \mathrm{~mW} \mathrm{~cm}^{-2}$ below the background intensity. The standard errors are indicated by black bars. b) Experimental data from positive perturbation (red plot), $\mathrm{I}=0.65 \mathrm{~mW} \mathrm{\textrm {cm } ^ { - 2 }}$ above the background intensity. The standard errors are indicated by black bars. c) Simulation of dark perturbation (black plot) of neurons fitted to data points and averaged (gray plot) from the experiment. The plotted data set (c) is from ref. [42]

FIG. 2.11: PRC with different positive perturbation sizes. a) Data points (blue circles) and averaged points (red plot) from experiment with positive perturbation, $\mathrm{I}=0.65 \mathrm{~mW} \mathrm{~cm}^{-2}$. b) Data points (blue circles) and averaged points (red plot) from experiment with positive perturbation $\mathrm{I}=0.19 \mathrm{~mW} \mathrm{~cm}^{-2}$

FIG. 3.1: Phase response curves. a) Positive perturbations result in a PRC with only a positive slope, which can be seen over the last half of the cycle. b) Negative perturbations result in a PRC with positive and negative slopes, where the first half of the cycle exhibits a small positive slope

FIG. 3.2: Mean signals. a) A time series showing a noisy mean signal (black) on top of four individual oscillators with non-coherent phases. b) A mean signal (black) of four individual oscillators that are partially synchronized and showing some degree of phase coherence. c) A mean signal (black) of four individual oscillators that are fully synchronized, showing phase coherence. 
FIG. 3.3: Order parameter $r$ indicating the degree of a phase coherence. a) Order parameter (red) near 0 indicates little coherence in the phases. b) Fluctuations of the order parameter (red) to higher values indicate a partially synchronized state. c) Order parameter (red) of 1 indicates complete synchronization

FIG. 3.4: Mean intensity (black) and the order parameter (red) from antiphase synchronization to complete in phase synchronization. The system shows the evolution from antiphase synchronization to partial synchronization to in phase synchronization. The order parameter alone cannot distinguish the antiphase case from the non-coherent case

FIG. 3.5: Oscillators at their natural frequency with no external signal. a) The individual time series of 30 oscillators showing their natural oscillations. b) A histogram showing the distribution of the natural periods of 30 oscillators

FIG. 3.6: Periodic positive pulses with amplitude $\mathrm{I}=0.56 \mathrm{~mW} \mathrm{~cm}^{-2}$ above the background intensity. The perturbations are introduced at $\mathrm{t}=126.0 \mathrm{~s}$ and are shown in gray scale $\ldots . . . . .71$

FIG. 3.7: Adaptive periodic positive pulses to the mean periods of five control oscillators. The figure shows one control bead representing the mean natural period of the system, $\mathrm{T}_{0}$. The pulse signals are applied with the adaptive period $\mathrm{T}_{\mathrm{sig}}=\mathrm{T}_{0}-9.0 \mathrm{~s}$

FIG. 3.8: Periodic negative pulses with amplitude $\mathrm{I}=1.67 \mathrm{~mW} \mathrm{~cm}^{-2}$ below the background intensity introduced at $\mathrm{t}=1200.0 \mathrm{~s}$ after the positive pulses, $\mathrm{I}=0.56 \mathrm{~mW} \mathrm{~cm}^{-2}$, above the background intensity

FIG. 3.9: Signal synchronized to external periodic positive pulses in 30 oscillators. a) Positive light pulse (blue) introduced at $\mathrm{t}=126.0 \mathrm{~s}$, with a period faster than the mean period of five control oscillators by $9.0 \mathrm{~s}$ with a width of $9.0 \mathrm{~s}$. The mean signal (black) 
is regular and synchronized to the period of the positive pulse. b) Individual time series of ten representative oscillators, which show high-phase coherence. c) The order parameter (red) is close to 1.0, indicating a high degree of synchronization. System of 30 micro-oscillators loaded with $\mathrm{Ru}(\mathrm{bpy})_{3}^{2+}$ catalyst, with a mean natural period of 79.9 s. The background intensity is $\mathrm{I}_{0}=1.67 \mathrm{~mW} \mathrm{~cm}^{-2}$. BZ reaction mixture composition: malonic acid, $[\mathrm{MA}]=0.08 \mathrm{M}$; sodium bromide, $[\mathrm{NaBr}]=0.02 \mathrm{M}$; sulfuric acid, $\left[\mathrm{H}_{2} \mathrm{SO}_{4}\right]=0.78 \mathrm{M}$; sodium bromate, $\left[\mathrm{NaBrO}_{3}\right]=0.48 \mathrm{M}$

FIG. 3.10: Positive square wave-forcing signal followed by negative pulses during the first half of the period. a) The positive light pulse signal (blue) was introduced at $\mathrm{t}=126.0 \mathrm{~s}$, while the negative light pulse signal was introduced at $\mathrm{t}=1200.0 \mathrm{~s}$, immediately after the positive pulses and lasting for the first half of the mean period. The mean signal (black) after $1200.0 \mathrm{~s}$ was attenuated and irregular, indicating desynchronization. b) Individual time series of ten representative oscillators. c) The order parameter (red) is reduced after introducing the negative pulse, indicating desynchronization. System of 30 micro-oscillators loaded with $\mathrm{Ru}(\mathrm{bpy}){ }_{3}^{2+}$ catalyst, with a mean natural period of $78.7 \mathrm{~s}$. The background intensity is $\mathrm{I}_{0}=1.67 \mathrm{~mW} \mathrm{~cm}^{-2}$. The $\mathrm{BZ}$ reaction mixture composition is the same as in Figure 3.9.

FIG. 3.11: Square wave-forcing signal followed by dark pulses in the last half of the period.

a) The positive light pulse signal (blue) is introduced at $t=126.0 \mathrm{~s}$, while the negative light pulse signal is introduced at $\mathrm{t}=1200.0 \mathrm{~s}$, immediately after the positive pulses and lasting for the last half of the mean period. The mean signal (black) after $1200.0 \mathrm{~s}$ preserves regularity, indicating that the negative pulses are not able to desynchronize the oscillators. b) Individual time series of ten representative oscillators. c) The order 
parameter (red) remains close to 1.0 after introducing the negative pulse, indicating a synchronization state. The system is made up of 30 micro-oscillators loaded with $\mathrm{Ru}(\mathrm{bpy})_{3}^{2+}$ catalyst, with a mean natural period of $83.7 \mathrm{~s}$. The background intensity is $\mathrm{I}_{0}=1.67 \mathrm{~mW} \mathrm{~cm}^{-2}$. The $\mathrm{BZ}$ reaction mixture composition is the same as in Figure $3.9 \ldots .78$

FIG. 3.12: Periodic positive half-sine wave with amplitude of 100 on the gray scale, corresponding to $0.93 \mathrm{~mW} \mathrm{~cm}^{-2}$ above the background intensity, was introduced at $\mathrm{t}=$ 126.0 s. Periodic negative pulses with light intensity $\mathrm{I}=1.29 \mathrm{~mW} \mathrm{~cm}^{-2}$ below the background intensity were introduced after $1200.0 \mathrm{~s}$

FIG. 3.13: Half-sine wave forcing signal followed by negative pulses applied for the first half of the mean period with a $3.0 \mathrm{~s}$ offset from the half-sine wave signal. a) The positive half-sine wave light signal (blue) is introduced at $\mathrm{t}=126.0 \mathrm{~s}$, while the negative light pulse signal is introduced at $\mathrm{t}=1200.0 \mathrm{~s}$, with a $3.0 \mathrm{~s}$ offset from the end of the half-sine wave signal that lasted for the first half of the mean period. The mean signal (black) is slightly reduced after $1200.0 \mathrm{~s}$, indicating some desynchronization. b) Individual time series of ten representative oscillators, showing some loss of phase coherence among the oscillators. c) The order parameter (red) is slightly reduced after introducing the negative pulse, indicating some extent of desynchronization. The system is made up of 30 micro-oscillators loaded with $\mathrm{Ru}(\mathrm{bpy})_{3}^{2+}$ catalyst, with a mean natural period of 90.1 s. The background intensity is $\mathrm{I}_{0}=1.29 \mathrm{~mW} \mathrm{~cm}^{-2}$. The $\mathrm{BZ}$ reaction mixture composition is the same as in Figure 3.9

FIG. 3.14: Sine wave forcing signal followed by negative pulses for the first half of the mean period with a $6.0 \mathrm{~s}$ offset from the half-sine wave signal. a) A positive half-sine wave light signal (blue) introduced at $\mathrm{t}=126.0 \mathrm{~s}$, while the negative light pulse signal is 
introduced at $\mathrm{t}=1200.0 \mathrm{~s}$, with a $3.0 \mathrm{~s}$ offset from the end of the half-sine wave signal that lasted for the first half of the mean period. The mean signal (black) is reduced after $1200.0 \mathrm{~s}$, indicating desynchronization. b) Individual time series of ten representative oscillators show loss of phase coherence among oscillators. c) The order parameter (red) is reduced after introducing the negative pulse, indicating desynchronization. System of 30 micro-oscillators loaded with $\mathrm{Ru}(\mathrm{bpy})_{3}^{2+}$ catalyst, with a mean natural period of 62.4 s. The background intensity is $\mathrm{I}_{0}=1.29 \mathrm{~mW} \mathrm{~cm}^{-2}$. The $\mathrm{BZ}$ reaction mixture composition is the same as in Figure 3.9

FIG. 3.15: Positive square-wave forcing signal followed by negative pulses applied from the beginning of the phase cycle of the synchronized mean signal of eight oscillators. a) The positive light pulse signal (blue) is introduced at $t=160000$ time steps, while the negative light pulse signal is introduced at $\mathrm{t}=521000$ time steps immediately after the positive pulses. The mean signal (black) decreases and becomes irregular after 521000 time steps, indicating desynchronization. b) Individual time series of the eight oscillators shows phase incoherence. c) The order parameter (red) is reduced after introducing the negative pulse, indicating desynchronization

FIG. 3.16: Positive square-wave forcing signal followed by negative pulses applied at the end of the phase cycle of the synchronized mean signal of eight oscillators. a) The positive light pulse signal (blue) is introduced at $\mathrm{t}=160000$ time steps, while the negative light pulse signal is introduced at $t=521000$ time steps, before the positive pulses at the end of the phase cycle. The mean signal (black) preserved its regularity after 521000 time steps, indicating that these negative pulses are not able to desynchronize the signal. b) Individual time series of the eight oscillators maintains their phase coherence. c) The 
order parameter (red) continues to be one after introducing the negative pulse, indicating maintenance of the synchronized signal

FIG. 4.1: Two typical noise signals. a) White noise with a fast signal comprising all frequencies. b) Colored noise with a slow signal having dominant frequencies

FIG. 4.2: A Fourier transform power spectrum shows lower frequency domains of the colored noise signal

FIG. 4.3: Periodic negative pulses with amplitude $\mathrm{I}=1.29 \mathrm{~mW} \mathrm{~cm}^{-2}$ below the background intensity introduced at $\mathrm{t}=126.0 \mathrm{~s}$ along with the colored noise forcing.

FIG. 4.4: Oscillators synchronized by external colored noise. a) The colored noise light signal (blue) is introduced at $\mathrm{t}=126.0 \mathrm{~s}$. The mean signal (black) shows regions of enhancement, indicating a noise-induced synchronization phenomenon. b) Individual time series of ten representative oscillators, showing moderate coherence in phase. c) The mean of the order parameter (red) is 0.64 , indicating a moderate degree of synchronization. A system of 30 micro-oscillators loaded with $\mathrm{Ru}(\mathrm{bpy})_{3}^{2+}$ catalyst, with a mean natural period of $63.7 \mathrm{~s}$. The background intensity is $\mathrm{I}_{0}=1.29 \mathrm{~mW} \mathrm{~cm}^{-2}$. The $\mathrm{BZ}$ reaction mixture composition: malonic acid, $[M A]=0.08 \mathrm{M}$; sodium bromide, $[\mathrm{NaBr}]=0.02 \mathrm{M}$; sulfuric acid, $\left[\mathrm{H}_{2} \mathrm{SO}_{4}\right]=0.78 \mathrm{M}$; sodium bromate, $\left[\mathrm{NaBrO}_{3}\right]=0.48$ M.

FIG. 4.5: Intrinsic oscillations of uncoupled BZ oscillators. a) The oscillators are maintained at a constant background light intensity. The mean signal (black) shows a noisy signal, indicating no synchronization. b) Individual time series of ten representative oscillators show non-coherent phases. c) The mean of the order parameter (red) is 0.14 , indicating no synchronization. System of 30 micro-oscillators loaded with $\mathrm{Ru}(\mathrm{bpy})_{3}^{2+}$ catalyst, 
with a mean natural period of $85.8 \mathrm{~s}$. The background intensity is $\mathrm{I}_{0}=1.29 \mathrm{~mW} \mathrm{~cm}$. The $\mathrm{BZ}$ reaction mixture composition is the same as in Figure 4.4

FIG. 4.6: Fourier transform power spectra for noise-driven and autonomous BZ systems. a) The power spectrum of the mean signal from a noise-driven BZ system shows a narrow range of dominate frequencies, indicating noise-induced synchronization. b) The power spectrum of the mean signal from intrinsic oscillations of uncoupled BZ oscillators shows the lack of a dominant frequency, indicating no synchronization when no forcing signal is applied.

FIG. 4.7: Uncoupled BZ oscillators subjected to both negative pulses and colored noise. a) Negative pulses (red) and noise signals introduced at $\mathrm{t}=126.0 \mathrm{~s}$ (blue). The mean signal (black) shows a noisy signal, indicating desynchronization. b) The time series of ten representative oscillators show non-coherent phases. c) The mean order parameter (red) is 0.24 , which is low and indicates desynchronization. System of 30 micro-oscillators loaded with $\mathrm{Ru}(\mathrm{bpy})_{3}^{2+}$ catalyst, with a mean natural period of $86.0 \mathrm{~s}$. The background intensity is $\mathrm{I}_{0}=1.29 \mathrm{~mW} \mathrm{~cm}^{-2}$. The $\mathrm{BZ}$ reaction mixture composition is the same as in Figure 4.4

FIG. 4.8: Oscillators synchronized by external positive pulses in the presence of noise. a) Positive pulses (red) and colored noise light signal (blue) introduced at $\mathrm{t}=126.0 \mathrm{~s}$. The mean signal (black) shows enhancements and regular signal, indicating synchronization. b) The time series of ten representative oscillators show aligned coherence in phase. c) The mean of the order parameter (red) is 0.85 , indicating a high degree of synchronization. System of 27 micro-oscillators loaded with $\mathrm{Ru}(\mathrm{bpy})_{3}^{2+}$ catalyst, with 
mean natural period of $69.4 \mathrm{~s}$. The background intensity is $\mathrm{I}_{0}=1.29 \mathrm{~mW} \mathrm{~cm}^{-2}$. The BZ

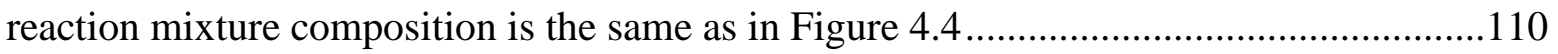

FIG. 5.1: A bidirectional 10-node network. Nodes that are the same color represent a symmetry cluster, and links (gray lines) represent the network connectivity......

FIG. 5.2: Phase-lag symmetry cluster synchronization. a) A reduced network topology showing the number of symmetry clusters represented by different colors, a simplification of Figure 5.1. b) Network topology showing three synchronization groups, each with simultaneously firing nodes, indicated by different colors (red, blue, and green). The phase leader is node 1 (red), which corresponds to the green node in the symmetry representations of the network in Figure 5.1. c) A plot of the natural period of each oscillator without coupling. d) The recorded gray-scale values for each node ordered by its connectivity to the phase leader. The wave pattern of simultaneously firing oscillators in the synchronization groups, which are separated by red lines. The color coding for the symmetry clusters as in Figure 5.1 and for the synchronization groups as in (b). The node index is numbered according to network representation in (b). e) Order parameter after coupling was introduced at $t=200.0 \mathrm{~s}$. The order parameter of all nodes (black plot) shows no overall zero-lag phase synchronization in the system, whereas the order parameter for each synchronization group is close to 1.0 , indicating phase synchronization. The color coding is as in (b). f) A linear fit of oscillator phases against their synchronization group index, showing phase-lag synchronization with a constant phase shift; color coding is as in (b). A system of 10 micro-oscillators loaded with $\mathrm{Ru}(\mathrm{bpy}){ }_{3}^{2+}$ catalyst, with a mean natural period of $35.04 \mathrm{~s}$. The coupling strength is $\mathrm{K}=2.0$. The $\mathrm{BZ}$ reaction mixture composition: malonic acid, $[M A]=0.08 \mathrm{M}$; sodium 
bromide, $[\mathrm{NaBr}]=0.02 \mathrm{M}$; sulfuric acid, $\left[\mathrm{H}_{2} \mathrm{SO}_{4}\right]=0.78 \mathrm{M}$; sodium bromate, $\left[\mathrm{NaBrO} \mathrm{O}_{3}\right]$ $=0.48 \mathrm{M}$

FIG. 5.3: Phase-lag synchronization. a) The network topology showing four groups of simultaneous firing nodes, indicated by different colors (red, blue, green, and magenta). b) A plot of the natural periods of all oscillators with no coupling. c) The recorded grayscale values for each node, as ordered by connectivity to the phase leader, showing a wave pattern of the synchronization groups, separated by red lines. The phase leader is node 1 (red), which corresponds to one of the nodes forming a symmetry cluster, but not a complete cluster. The color coding for the symmetry clusters is as in Figure 5.1. The node index is numbered and colored according to the synchronization groups in (a). d) Order parameter after the coupling introduced at $\mathrm{t}=200.0 \mathrm{~s}$ indicating in-phase synchronization groups, where the order parameter is close to 1.0 for each group. The global order parameter of all nodes (black plot) shows no overall zero-lag phase synchronization in this system. The color code is as in (a). e) A linear fit of oscillator phases against the synchronization group index, showing phase-lag synchronization with a constant phase shift; color coding is as in (a). A system of 10 micro-oscillators loaded with a $\mathrm{Ru}(\mathrm{bpy})_{3}^{2+}$ catalyst, with a mean natural period of $50.65 \mathrm{~s}$. The coupling strength is $\mathrm{K}=2.0$. The $\mathrm{BZ}$ reaction mixture composition is the same as in Figure 5.2 2.....130

FIG. 5.4: Partial frequency synchronization. a) The 10-node network topology of all permutation symmetry clusters shown with different colors. b) A plot of the periods of all beads after switching on coupling, showing splitting into two synchronized periods, which indicates partial frequency synchronization. c) The recorded gray-scale values for each node, as ordered by connectivity to the phase leader. Deviation of phase-lag 
patterns in nodes 8 and 9 is observed. The node index is numbered and colored according to network representation in (a). A system of 10 micro-oscillators loaded with $\mathrm{Ru}(\mathrm{bpy}){ }_{3}^{2+}$ catalyst, with a mean natural period of $55.68 \mathrm{~s}$. The coupling strength is $\mathrm{K}=$ 0.60. The $\mathrm{BZ}$ reaction mixture composition is the same as in Figure 5.2

FIG. 5.5: Incoherent oscillation. a) The 10-node network topology of all permutation symmetry clusters with different colors. b) A plot of the periods of all beads after switching on coupling. A wide distribution of periods indicates no synchronization. c) The recorded gray-scale values for each node, showing an irregular pattern. The node index is numbered and colored according to network representation in (a). A system of 10 micro-oscillators loaded with $\mathrm{Ru}(\mathrm{bpy}) 3_{3}^{2+}$ catalyst, with a mean natural period of $55.97 \mathrm{~s}$. The coupling strength is $\mathrm{K}=0.05$. The $\mathrm{BZ}$ reaction mixture composition is the same as in Figure 5.2 


\section{List of Tables}

Table 2.1: The Detailed Zhabotinsky, Buchholtz, Kiyatkin, and Epstein (ZBKE) Mechanism

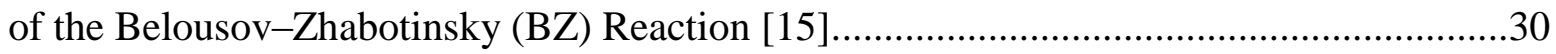

Table 2.2: The Zhabotinsky, Buchholtz, Kiyatkin, and Epstein Model. ...................................34

Table 2.3: Scaling Parameters for the Dimensionless Zhabotinsky, Buchholtz, Kiyatkin, and Epstein Model [24].

Table 2.4: Parameter Values for the Two-Variable Zhabotinsky, Buchholtz, Kiyatkin, and Epstein Simulation [26].

Table 4.1: Data of Mean Order Parameters Values in Each Set of Experiments.

Table 4.2: Calculated t-test from Two Different Sets of Experiments $(\mathrm{A}=$ noise-forcing experiments; $\mathrm{B}=$ negative pulse and noise-forcing experiments; $\mathrm{C}=$ no forcing experiments). 


\section{Chapter 1}

\section{Synchronization}

\subsection{Introduction}

Synchronization phenomena are universal, and refer to the adjustment of the rhythms of interacting oscillators. This occurs when all oscillators in a system spontaneously adjust their individual frequencies to a common frequency from their intrinsic initial state. Synchronization is essential for many functions in living systems, including intellectual, physiological, behavioral, and social activities. In the seventeenth century, synchronization was first studied by the Dutch physicist Christiaan Huygens with two pendulum clocks, which were hung from the same support and exhibited out-of-phase synchrony [1]. The mutual synchronization in Huygens' clocks resulted from their mutual coupling through the beam support [2]. Since then, there has been great interest in studying synchronization in many physical and biological systems that reveal patterns of spontaneous rhythm alignment.

A striking biological example of synchronization is found in Southeast Asia fireflies, in which a group of males light up in unison because of visual feedback from neighboring fireflies [3]. Moreover, the phenomenon can be reproduced in laboratory experiments with an artificial light stimulus, which induces synchronous flashing in populations of fireflies [4]. When the rhythm adjustment is toward a specific source of oscillation, such as a pacemaker or a common external stimulus, the synchrony is referred to as entrainment. An example is the entrainment of circadian systems to environmental signals. Biological clocks in humans, animals, and plants are entrained by the 24 hour periodic perturbation of the day-night cycle. The effect of light in resetting timing phases in circadian clocks on physiological function in plants was first 
recognized by Erwin Bünning in 1936 [5], which was followed by his findings of circadian clocks in insects in 1960 [6]. This concept was developed further, with manipulation of oscillations by visible light in 1983 in an oscillatory chemical system [7], where the lightsensitive form of the Belousov-Zhabotinsky (BZ) reaction was introduced. The synchronization of BZ oscillators is illustrated in Figure 1.1. Applying light for feedback control of photosensitive oscillators provides the potential to explore the synchronization behavior of coupled and uncoupled oscillators.

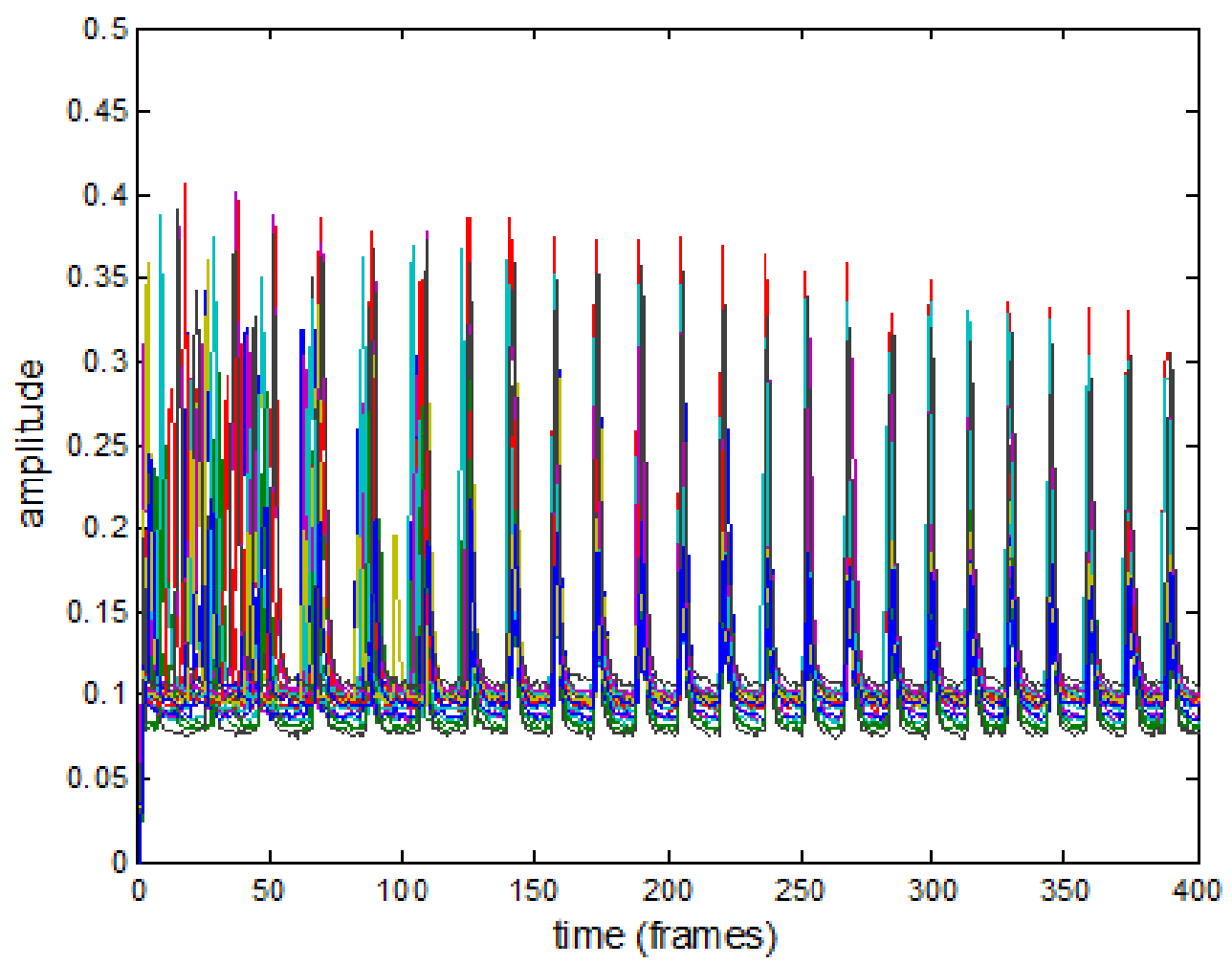

FIG. 1.1: Individual time series showing synchronization in the chemical Belousov-Zhabotinsky (BZ) system. BZ reaction mixture composition: malonic acid, $[M A]=0.08 \mathrm{M}$; sodium bromide, $[\mathrm{NaBr}]=$ $0.02 \mathrm{M}$; sulfuric acid, $\left[\mathrm{H}_{2} \mathrm{SO}_{4}\right]=0.78 \mathrm{M}$; sodium bromate, $\left[\mathrm{NaBrO}_{3}\right]=0.48 \mathrm{M}$. System of 20 microoscillators loaded with $\mathrm{Ru}(\mathrm{bpy})_{3}^{2+}$ catalyst. 


\subsection{Biology Background}

Physiological rhythms are essential to all living organisms, including the sleep-wake cycle [8], heart beat [9], respiration [10], and many other complicated intellectual, physiological, and social activities. These bodily rhythms reflect the interaction of many physiological oscillators in response to changes in their inner and outer environments, and thus fluctuate over time resulting in their intended action or activity [11]. Any deviations of a biological system from its normal rhythms may cause behavioral disorders or diseases, with many studies suggesting that disruption of neuronal synchrony is associated with dysfunctions found in Autism, Schizophrenia, Epilepsy, Alzheimer's disease, and Parkinson's disease (PD) [12]. Tremors in PD are suggested to emerge from a spontaneous increase of neural activity that leads to synchronized oscillations [13]. These abnormal synchronous oscillations are accompanied by defective stimulation of the motor cortical system by neuronal circuits responsible for controlling body movements [14]. These neurons comprise a group of interconnected areas deep inside the brain termed the basal ganglia, including the substantia nigra, subthalamic nucleus, globus pallidus internal and external, and putamen and caudate nucleus, as shown in Figure 1.2 [15]. The basal ganglia plays a role in controlling the neural feedback loop by providing essential activation or inhibition [16]. Therefore, the basal ganglia has been a target for neurosurgical therapies using various techniques including ablative surgeries and, more recently, safer procedures for deep brain stimulation (DBS). DBS involves the use of a neurosurgical pulse generator, with the main objective of inducing a desynchronized state among the neurons to reestablish normal information flow within their feedback loops for proper physiological activities [16]. 


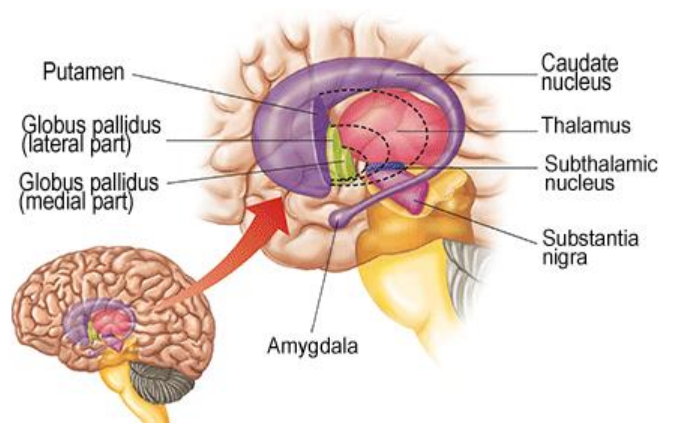

FIG. 1.2: Basal ganglia structures. Illustrated image is from ref. [15].

There is now widespread interest in the use of electrical stimulation of the brain for neurobehavioral studies and as a treatment for neurological diseases. In 1874, Robert Bartholow pioneered the use of direct brain stimulation in the cerebral cortex of humans [17]. In 1947, a three-dimensional scanning technique using stereotactic devices to perform deep brain lesioning surgery was reported. However, lesioning surgery (i.e., tissue freezing, burning, or treating with chemical substances) is accompanied by irreversible side effects [18]. In 1963, Albe-Fessard reported that high frequency stimulations of $100-200 \mathrm{~Hz}$ in humans could reduce tremors [19]. Permanent implantation of electrodes in deep brain structures as a therapeutic treatment was performed, from which the modern technique of DBS was developed [19].

DBS was first introduced in 1987 in France by Benabid and co-workers for controlling movement and tremor [20]. DBS involves the implantation of electrodes with stereotactic technology into a selected structure of deep brain regions to treat various dysfunctions associated with neural diseases, as illustrated in Figure 1.3 [21]. For example, the two most common sites for controlling PD are the subthalamic nucleus and globus pallidus internal [16], while the thalamus is typically targeted to control essential tremor [21]. A periodic train of pulses emitted from a pulse generator is transmitted by electrodes. The generator is placed under the skin near the clavicle of the patient and has an on/off switch [21]. 
a)

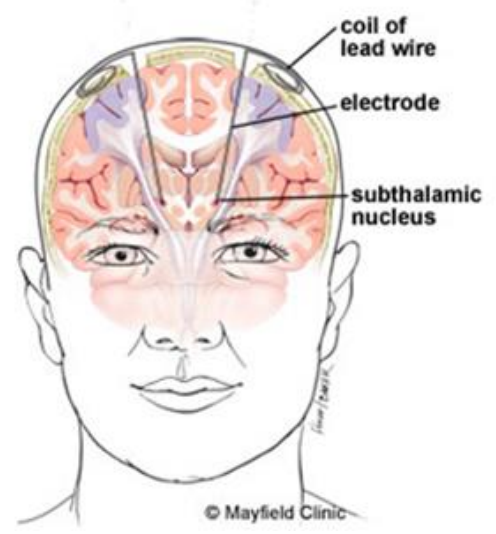

b)

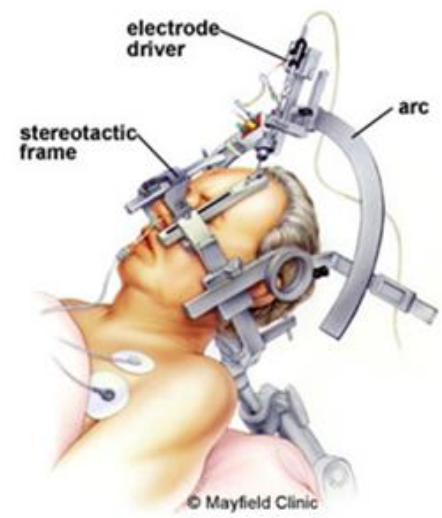

FIG. 1.3: Deep brain stimulation. a) A cross section of the brain shows the electrode placement in the subthalamic nucleus for deep brain stimulation for Parkinson's disease. b) Illustration of the stereotactic device. Illustrated images are from ref. [21].

DBS has promising potential for controlling epileptic seizures [22] and PD [23, 24], and has been tried in several animal models $[25,26]$. In 2002, DBS was approved to treat PD by the Food and Drug Administration after its initial approval to treat essential tremors in 1997 [27]. DBS had now become an essential clinical tool for improving the quality of life for patients with neurological disorders. However, despite the extensive clinical use of DBS, its therapeutic mechanism and pathology for relieving disease symptoms remains controversial. Computational models using nonlinear dynamics have been developed in an attempt to improve the mechanistic understanding of DBS. There are two main hypotheses of the therapeutic mechanism of DBS in these models. Some computational models of DBS have a stimulus entrainment mechanism [28, 29], while an alternative mechanism involves a disruption of the pattern of neural synchrony [30]. A model has been developed for a population of coupled oscillators in the presence of noise. The difficulty in developing and testing models of biological systems has led to alternative experimental systems that have features resembling neural relaxation oscillators, such as the BZ chemical reaction described in Section 1.3. 


\subsection{Chemistry Background: The BZ Reaction}

Oscillations can be observed in a wide range of biological, physical, and chemical systems, such as in heart pacemakers, electronic circuits that generate radio-frequency power, and the BZ chemical reaction. The $\mathrm{BZ}$ reaction is a catalyzed redox reaction that gives rise to oscillations and supports wave propagation. The Russian biochemist Boris Pavlovich Belousov was the first to observe the oscillatory behavior of this reaction in a stirred homogenous solution. He wrote an unpublished manuscript in 1951 on his discovery, which was finally published in 1981, eleven years after his death [31]. A graduate student, Anatol Zhabotinsky, studied and modified the system in 1961, and published the results in English in 1968 [32]. The BZ reaction has been used extensively for modeling complex biological oscillatory systems.

Self-sustained oscillatory systems maintain their natural periodic rhythm and return back to their intrinsic rhythm following small perturbations [2]. The graphical representation of selfsustained oscillations is depicted as a limit cycle oscillator (attractor), which is a closed orbit/trajectory where all variables periodically repeat themselves each cycle [33]. At least two variables are required to describe the waveform of an oscillation. In Figure 1.4, two variables form the coordinates of the phase plane to represent the limit cycle. If the concentration of one variable increases and decreases rapidly while the other variable slowly relaxes, then a relaxation oscillator is obtained [33]. The BZ chemical reaction can generate spontaneous relaxation oscillations in which the shape of an oscillation appears as a sequence of spikes, as shown in Figure $1.5 \mathrm{a}, \mathrm{b}$. These oscillations are similar to the spikes seen during neuronal firing. In this process, the intracellular potential variable slowly reaches a threshold voltage difference between the inside and outside of the neuronal cell. Thus, a rapid spike occurs with the discharging of the cell and the process repeats, as shown in Figure 1.5c [34]. 


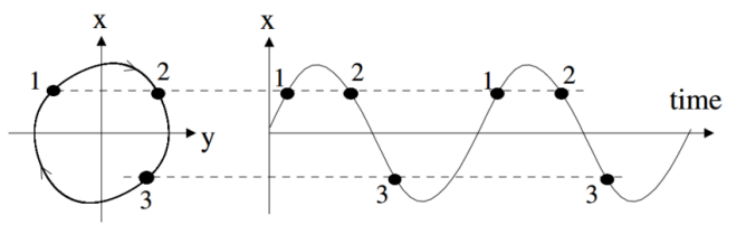

FIG. 1.4: A limit cycle curve of periodic oscillations in the phase plane and the corresponding time series plot. Points with the same numbers are of the same phase and are repeated for each period or cycle. Schematic diagram is from ref. [2].

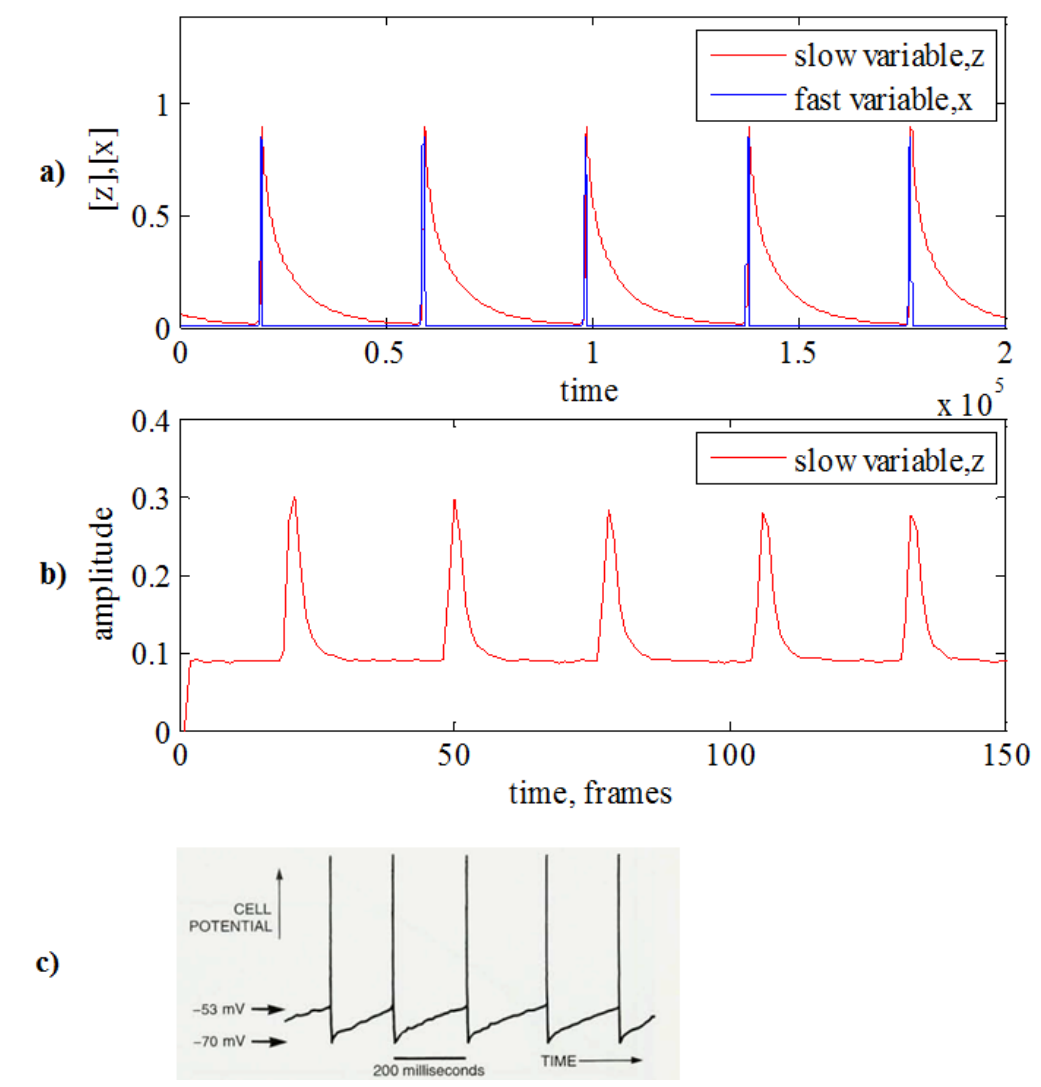

FIG. 1.5: Relaxation oscillators. a) A relaxation Belousov-Zhabotinsky (BZ) oscillation from a model simulation with two variables, $\mathrm{x}$ and $\mathrm{z}$, where $\mathrm{x}$ represents the fast variable, bromous acid, and $\mathrm{z}$ represents the slow variable, the catalyst. b) A relaxation BZ oscillation shown in the catalyst concentration in a BZ reaction experiment. c) Rapid neural firing generates a spike each time the cell potential slowly reaches $-53 \mathrm{mV}$, and the cell then discharges and is reset to $-70 \mathrm{mV}$. Schematic diagram is from ref. [34]. 
In the $\mathrm{BZ}$ reaction, the oscillations of the system are observed as a periodic color change. The main reactants are malonic acid, bromate, sulfuric acid, and a catalyst, such as ferroin $\left(\mathrm{Fe}(\mathrm{phen})_{3}^{2+}\right)$ or tris(bipyridine)ruthenium(II) $\left(\mathrm{Ru}(\mathrm{bpy})_{3}^{2+}\right)$. The photosensitive ruthenium catalyst can be used to induce or suppress oscillations by controlling the light intensity imposed on the system. The catalyst in the reaction serves as an oscillation indicator that changes its redox state and color periodically depending on the state of the system. The resulting intermediates, bromous acid as the autocatalyst and bromide as the inhibitor, act to regulate the oscillations in the system. Each intermediate, along with the catalyst, is represented by a firstorder ordinary differential equation as seen in the BZ kinetic models, the Oregonator [35] and the modified Zhabotinsky, Buchholtz, Kiyatkin, and Epstein (ZBKE) [36] models. This reaction with its available models has been widely used by chemists and physicists to study temporal and spatiotemporal dynamics.

The $\mathrm{BZ}$ reaction has been studied in gels [37, 38], droplets of aqueous solution in oil media [39], and with catalyst-loaded cation exchange beads [40-43], and is widely used to examine the synchronization of coupled oscillators. The photosensitive form of the BZ reaction, where ruthenium (II) is the catalyst, has been increasingly used to explore the nonlinear dynamics of coupled and uncoupled oscillators. We used the features of the photosensitive discrete oscillator system to mimic neuronal behavior with external forcing of uncoupled oscillators. More details on the photosensitive BZ model and the experimental setup are discussed in Chapter 2. In Chapters 3 and 4, the desynchronization effects of dark periodic perturbations in BZ photochemical oscillators coupled by periodic pulses or colored noise were verified experimentally. The results of initiating desynchronization by a regular stimulus were in agreement with predictions of a theoretical model of DBS [30]. In Chapter 5, phase-lag 
synchronization in a network of discrete oscillators was explored, which has a different mechanism than the reaction-diffusion behaviors seen in continuous media [44, 45], quasicontinuous media [43, 46-48], and discrete media with distance dependent coupling [49], and has not been observed in populations of discrete non-diffusively coupled BZ oscillators [50].

\subsection{Synchronization Setup}

Synchronization is observed when mutual coupling is established among oscillators with two-way interactions. In addition, it can be established by exerting external forcing on oscillators that show one-way interactions. The remainder of this chapter describes these two processes.

\subsubsection{Synchronization via Mutual Coupling}

Oscillators of naturally distributed frequencies may undergo weak interactions, or coupling, and as a result they may become synchronized. The resulting collective behavior depends on the coupling strength and the frequency distribution. When an oscillatory system has no coupling, each oscillator oscillates with its own natural frequency. However, when the coupling is large, the variations of the natural frequencies become negligible and the system is synchronized [2].

The intensity of light, for example, is a form of coupling strength in the synchronization of fireflies. Each firefly communicates and responds to all others in the group based on the emitted light pulses by the whole population. This interaction is called all-to-all coupling, where all oscillators interact with each other until the entire population adjusts to a common period or frequency, termed frequency locking or frequency synchronization. Synchronization occurs when the coupling among oscillators overcomes the heterogeneity of the system. 
Frequency synchronization may be followed by phase locking. The phase is the quantity that locates cyclic oscillators at each repeated period. Figure 1.6 shows the common phase locking cases include in-phase synchronization and antiphase synchronization, where the phase shift is constant.
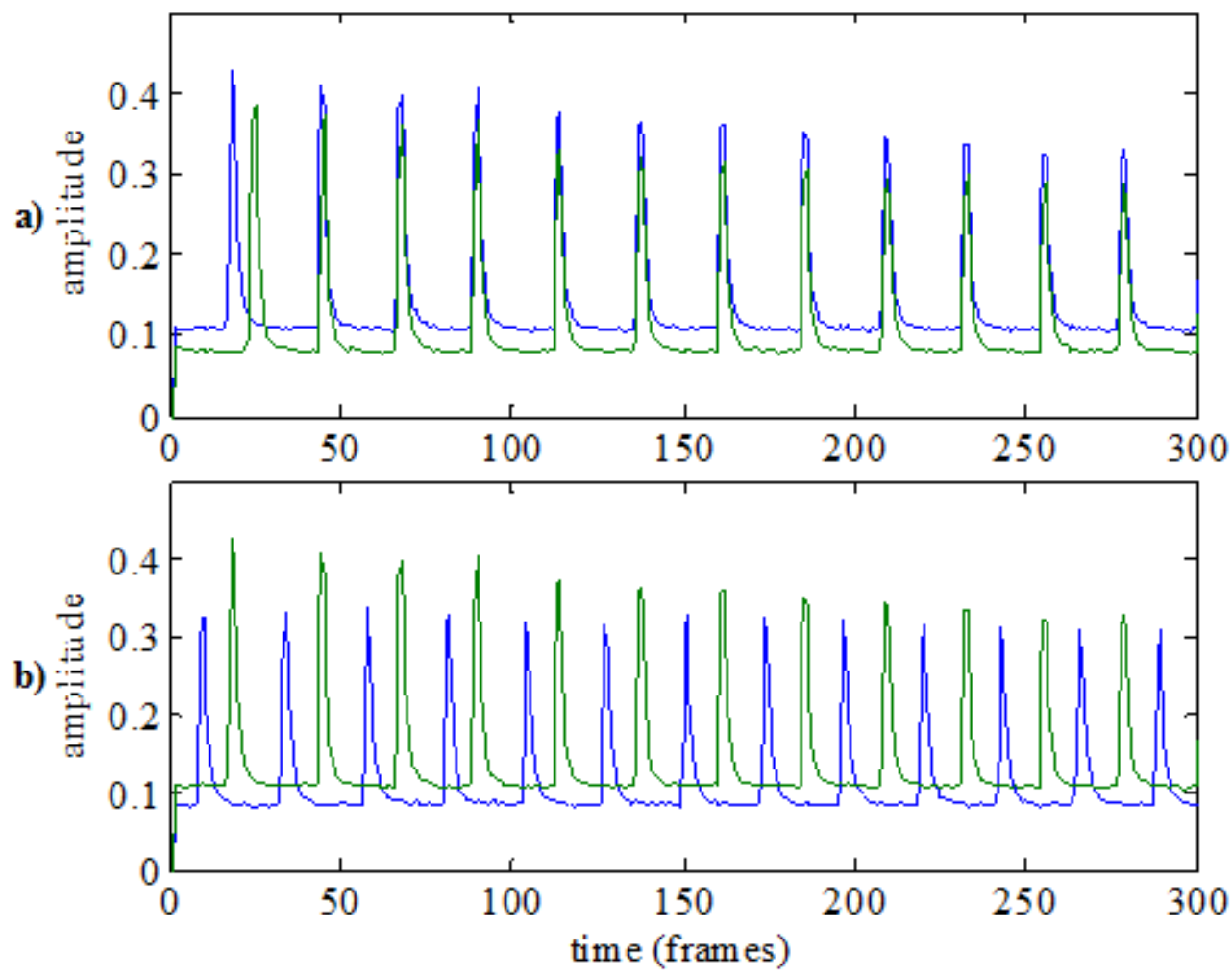

FIG. 1.6: Two main types of synchronization. a) In-phase synchronization of two oscillators. b) Antiphase synchronization of two oscillators.

An oscillatory medium can be continuous, quasi-continuous, or discrete. A discrete system can be arranged in chain, ring, lattice network structures, or many possible irregular network structures. These systems can have different coupling structures, and synchronization depends on the nature of the interactions. A basic form occurs when each element interacts with all other elements, leading to all-to-all coupling. When each element only interacts with its neighbors or with extended neighbors, then the coupling is considered to be local [43] or non-local [50], 
respectively. Many studies have focused on the dynamical behavior of oscillators as a function of coupling strength. Fukuda et al. [43] investigated synchronization dynamics in a chain of chemical bead oscillators $(0.90 \pm 0.01 \mathrm{~mm}$ diameter $)$ that were distributed spatially in one dimension, with a pacemaker oscillatory bead of a higher frequency. As the distance between the beads was decreased, more beads in the chain became entrained by the pacemaker until all of the beads were synchronized.

The Kuramoto model is a theoretical tool to describe spontaneous synchronization in a population of weakly coupled oscillators. The simple form of the phase equation for a population of $\mathrm{N}$ oscillators with a natural frequency distribution and all-to-all sinusoidal coupling is described as follows [51]:

$$
\dot{\theta}_{l}=\omega_{i}+\frac{\mathrm{K}}{\mathrm{N}} \sum_{j=1}^{\mathrm{N}} \sin \left(\theta_{j}-\theta_{i}\right), i=1,2, \ldots, \mathrm{N}
$$

where $\theta_{i}$ is the phase of oscillator $i$ with the natural frequency $\omega_{i}$. Oscillator $i$ is coupled to all other oscillators by the sine of the phase difference, $\sin \left(\theta_{j}-\theta_{i}\right)$, times the normalized coupling strength $\frac{\mathrm{K}}{\mathrm{N}}$. The order parameter $r$ is a quantity to measure the phase coherence of weakly interacting oscillators, where the phase of each oscillator tends toward the average phase $\psi$ and $0 \leq r \leq 1$. This can be calculated using the following equation [51]:

$$
r \mathrm{e}^{\mathrm{i} \psi}=\frac{1}{\mathrm{~N}} \sum_{j=1}^{\mathrm{N}} \mathrm{e}^{\mathrm{i} \theta_{j}}
$$

Multiplying the order parameter equation by $e^{-i \theta_{i}}$, and then substituting it into the phase equation, leads to the following governing equation for phase synchronization transition in terms of the order parameter [51]:

$$
\dot{\theta}_{\iota}=\omega_{i}+\mathrm{Kr} \sin \left(\psi-\theta_{i}\right), \quad i=1,2, \ldots, \mathrm{N}
$$


resulting in collective behavior that can vary from being completely synchronous $(r=1)$ to nonsynchronous $(r=0)$. Figure 1.7 shows the variation of coupling strength from weak to strong couplings, which leads to a phase synchronization transition at the threshold of critical coupling strength $\mathrm{K}_{c}$.

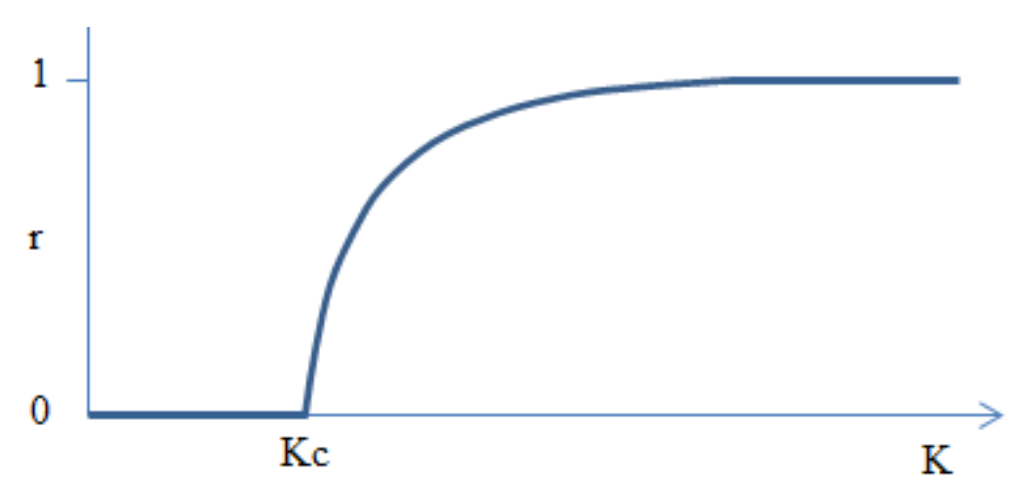

FIG. 1.7: Kuramoto model plot describing the order parameter $r$ to measure oscillator synchronization as a function of the coupling strength, $\mathrm{K}$. The transition to synchronization occurs at the critical threshold of the coupling strength $\mathrm{K}_{c}$.

The mechanism of Kuramoto spontaneous synchronous transition can be illustrated using the example of the firefly. A small group of nearby fireflies can exert mutual interactions and emit light in synchrony. Each individual firefly monitors photic inputs from neighbors, which it uses as feedback to adjust its own rhythm. The new adjusted inputs will enhance the overall maximum light intensities by a reduction in the frequency detuning. As a result, coupling becomes more pronounced and facilitates the synchrony transition. This step can be extended to the next neighbors until the entire population eventually exhibits synchronous flashing.

Partial synchronization, where only a portion of the oscillators is synchronized, can occur with intermediate coupling after the threshold. A synchronous cluster may occur when a portion of the system exhibits a stronger interaction than the other parts. Synchronized clusters in 
homogenous extended systems are seen with local and global feedback, where coupling among neighbors is stronger than with the rest of the medium [52]. Local coupling or local feedback represents any form of coupling that ensures that only neighbors or adjacent links in networks interact. Nishiyama and Eto investigated asymmetric coupling in a triangular arrangement of three beads of size 110,90 , and $90 \mu \mathrm{m}$ in diameter spaced at 170,150, and $140 \mu \mathrm{m}$, and found spontaneous switching between two out-of-phase oscillatory behaviors [49].

Another factor affecting the resulting collective behavior of the system involves heterogeneity in natural periods or frequencies of the oscillators, as shown in Figure 1.8. A tighter distribution has an earlier synchronization transition than a broader distribution. Based on the frequency distribution of a system, partial synchronization instead of complete synchronization may be obtained. Generally, a higher coupling is required to overcome heterogeneity in a system to reach a complete synchronization. Synchronized clusters can result from introducing heterogeneity in initial conditions [46] or in coupling [50]. In yeast cell studies, Weber et al. [53] reported that heterogeneous oscillatory yeast cells have a transition to cluster synchronization, each with the same frequency range, after a threshold of increasing population density. Taylor et al. [54] demonstrated evidence of phase cluster states, where groups of oscillators are both frequency and phase synchronized in stirred heterogeneous discrete BZ oscillators. Tinsley et al. [50] also observed phase clusters in heterogeneous coupling of discrete BZ oscillators. In network studies, where 'links' between network elements (nodes) replaces the notation of diffusively coupled systems, a pattern of synchronized clusters is also observed. Pecora et al. [55] reported synchronized clusters in an electro-optic network, with decreasing connections by removal of links of an all-to-all connected network. Different representations of an all-to-all network, bidirectional network, or unidirectional network are illustrated in Figure 
1.9. A distinct case is the unidirectional network, which leads to entrainment behavior, where one oscillator acts as an external force on the rest of the coupled oscillators. Synchronization by external forcing will be discussed in the following section.

a)

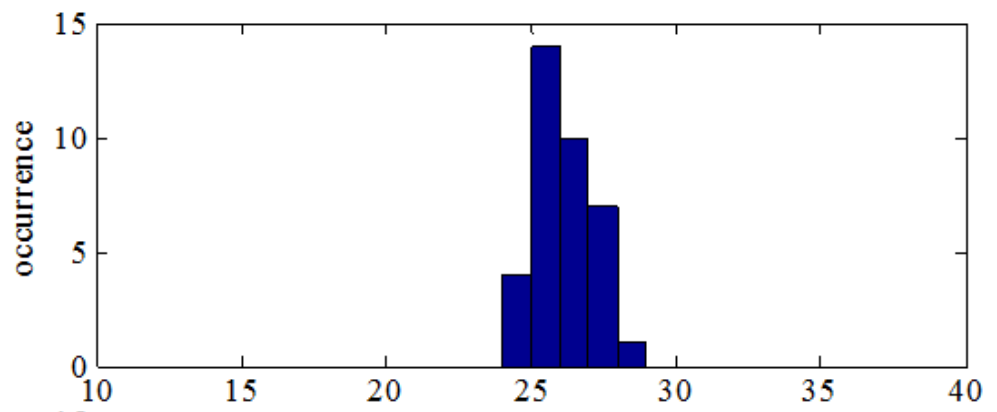

b)

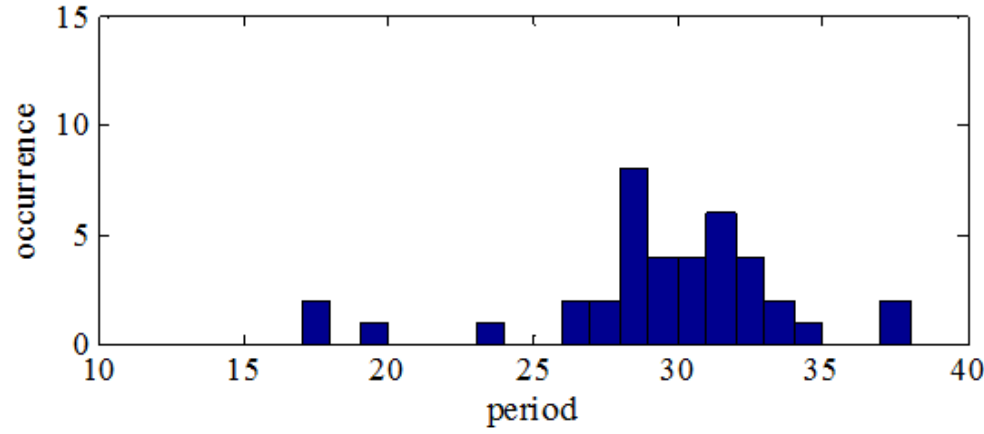

FIG. 1.8: Natural period distributions. a) A narrow distribution of natural periods. b) A wide distribution of natural periods.

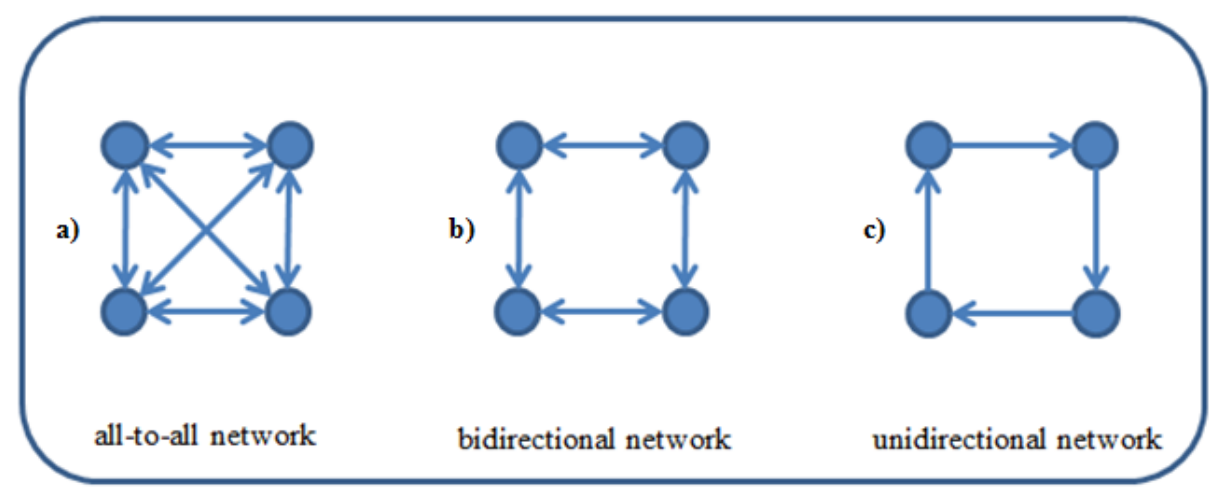

FIG. 1.9: Network connections. a) An all-to-all network, where each node is connected to all other nodes in the network. b) A bidirectional network, where pairs of oscillators influence each other equally. c) A unidirectional network, where the interaction is in one direction. 


\subsubsection{Synchronization via External Forcing}

Synchronization driven by external forcing represents a case of entrainment where the interaction between an oscillator and the external signal is unidirectional and non-mutual. An example is the biological clock in living systems entrained to the 24 hour light-dark cycle, where the circadian rhythms of cells are entrained to the external periodic stimulation originating from Earth's regular revolution around its axis $[56,57]$. Each weak external perturbation can cause a phase change, depending on the time of the perturbation in the oscillator cycle. Figure 1.10 describes a phase point on the limit cycle that moves to a new position of the perturbed state, while the amplitude relaxes back to the same amplitude [2]. A weak perturbation affects only the phase of the oscillators, but not the amplitude or shape of the limit cycle. To understand the influence of perturbations on these oscillations, the phase dynamics after perturbations are described using the Phase Response Curve (PRC) in Chapter 2.

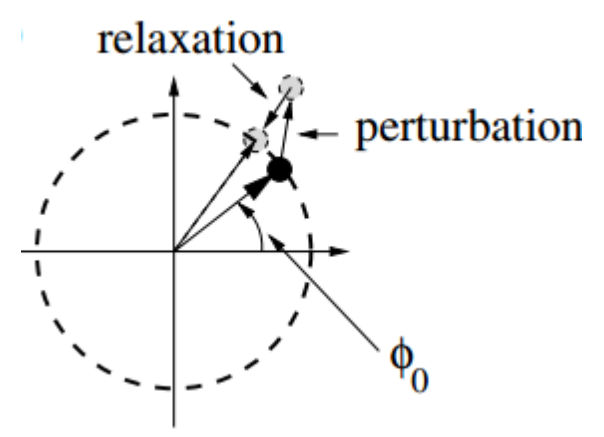

FIG. 1.10: Perturbation influence on oscillations. The external weak perturbation changes the phase point (filled circle) to a new position in the perturbed state (dashed circles). The initial phase of the oscillator is $\phi_{0}$. Schematic diagram is from ref. [2].

For frequency synchronization to occur with a weak external forcing, the difference in the frequency of the oscillators and the frequency of the forcing (i.e., detuning) should be small [2]. 
The relationship between forcing amplitude and frequency in a given detuning range is shown in Figure 1.11. The synchronization region is described by the gray triangle, known as an Arnold tongue [2].

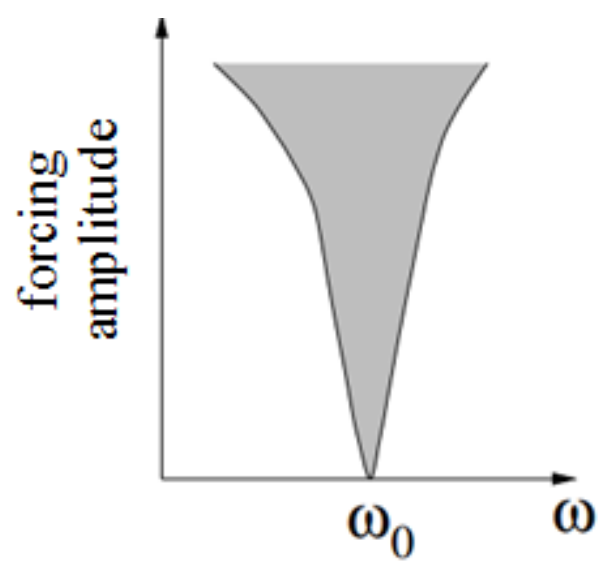

FIG. 1.11: The relationship between the forcing amplitude and frequency detuning, i.e., how much the external forcing frequency $\omega$ differs from the oscillator frequency $\omega_{0}$. The gray area reflects the Arnold tongue for the synchronization region. Schematic diagram adapted from ref. [2].

Different frequency-locking regimes can be observed depending on the frequency of the external force $\left(f_{e}\right)$ that varies as a $q: p$ ratio from the locked oscillatory frequency $\left(f_{l}\right)$ of the system, in which repeated $p$ firing events arise from each forcing frequency $q f_{e}$ [58]. The general synchronization formula is:

$$
q f_{e}=p f_{l}, \quad q \& p=1,2,3, \ldots
$$

In 1997, Petrov et al. [59] studied the external forcing on a light sensitive BZ reaction, and observed 1:1, 2:1, 3:1, and 3:2 regimes of frequency synchronization in a homogeneous BZ extended medium. Frequency synchronization can be accompanied by phase synchronization. In the case where the ratio is $1: 1$ and the system oscillates homogeneously to the frequency of the external forcing, then in-phase synchronization is observed. When the ratio is $2: 1$ and two 
batches in the system oscillate by a $\pi$ phase difference, then out-of-phase synchronization is seen. Other regions with different phase shift patterns are observed [60].

Phase synchronization refers to a constant phase shift maintained between the phase of the oscillator $\theta(t)$ and that of the external forcing $\theta_{e}(t)$. The constant phase shift is established after frequency synchronization, i.e., only within the regions in the Arnold tongue. The special case of phase locking with zero lag occurs when there is no mismatch of the frequency of the force and that of the oscillator. Phase synchronization occurs if the external forcing is of the correct amplitude and is applied at particular phases. Phase locking of biological oscillations by external periodic forcing is important for understanding many rhythmic biological functions. Applications include heart pacemakers [61, 62], mechanical breathing aids [10, 63], and stimulation of neuron firing $[18,23]$.

An irregular form of perturbation, noise, is used for studying synchronization in natural systems subject to noise and random fluctuations. Noise is shown to enhance order and coherent patterns and allow amplification of weak signals (termed stochastic resonance). Noise supported wave propagation in subexcitable BZ media has been studied in a photosensitive BZ extended solution, where stochastic resonance was observed [64-66]. Noise-enhanced phase synchronization of chaotic and uncoupled oscillators has been studied in other systems, but not in the BZ reaction. Examples include coupled chaotic electrochemical oscillators $[67,68]$ and uncoupled sensory neuronal oscillators of paddlefish [69]. Noise-induced synchronization has never been studied in BZ systems or other non-biological systems. Chapters 3 and 4 discuss the behavior of uncoupled BZ oscillators subjected to periodic forcing (pulses) and aperiodic forcing (noise) while stimulating these systems with dark periodic perturbations. 


\section{References}

[1] A. E. Bell, "The horologium oscillatorium of Christian Huygens." Nature 148, 245-248 (1941).

Online Version

[2] A. Pikovsky, M. Rosenblum, and J. Kurths, Synchronization: A universal concept in nonlinear sciences (Cambridge University Press, Cambridge, 2003).

Online Version

[3] J. Buck and E. Buck, "Mechanism of rhythmic synchronous flashing of fireflies. Fireflies of Southeast Asia may use anticipatory time-measuring in synchronizing their flashing." Science 159, 1319-1327 (1968).

Online Version

[4] J. Buck, E. Buck, J. F. Case, and F. E. Hanson, "Control of flashing in fireflies. V. Pacemaker synchronization in Pteroptyx cribellata.” J. Comp. Physiol. 144, 287-298 (1981).

Online Version

[5] D. S. Saunders, "Erwin Bunning and Tony Lees, two giants of chronobiology, and the problem of time measurement in insect photoperiodism.” J. Insect Physiol. 51, 599-608 (2005).

Online Version

[6] E. Bünning, "Circadian rhythms and the time measurement in Photoperiodism." Cold Spring Harb. Symp. Quant. Biol. 25, 249-256 (1960).

Online Version 
[7] V. Gáspár, G. Bazsa, and M. Beck, "The influence of visible-light on the BelousovZhabotinskii oscillating reactions applying different catalysts.” Z. Phys. ChemieLeipzig 264, 43-48 (1983).

Online Version

[8] S. H. Strogatz, "Human sleep and circadian rhythms: A simple model based on two coupled oscillators.” J. Math. Biology 25, 327-347 (1987).

Online Version

[9] R. Wilders and H. J. Jongsma, "Beating irregularity of single pacemaker cells isolated from the rabbit sinoatrial node.” Biophys. J. 60, 2601-2613 (1993).

Online Version

[10] G. A. Petrillo and L. Glass, "A theory for phase-locking of respiration in cats to a mechanical ventilator.” Am. J. Physiol. 246, R311-R320 (1984).

Online Version

[11] L. Glass, "Synchronization and rhythmic processes in physiology." Nature 410, 277284 (2001).

Online Version

[12] P. J. Uhlhaas and W. Singer, "Neural synchrony in brain disorders: Relevance for cognitive dysfunctions and pathophysiology.” Neuron 52, 155-168 (2006).

Online Version

[13] H. Bergman, A. Raz, A. Feingold, A. Nini, I. Nelken, D. Hansel, H. Ben-Pazi, and A. Reches, "Physiology of MPTP tremor." Mov. Disord. 13, 29-34 (1998).

Online Version 
[14] R. Levy, P. Ashby, W. D. Hutchison, A. E. Lang, A. M. Lozano, and J. O. Dostrovsky, "Dependence of subthalamic nucleus oscillations on movement and dopamine in Parkinson's disease.” Brain 125, 1196-1209 (2002).

Online Version

[15] R. Joseph, Basal Ganglia, Striatum, Thalamus: Caudate, Putamen, Globus Pallidus, Limbic Striatum, Brainstem, Parkinson's Disease, Alzheimer's Disease, Psychosis, Schizophrenia, Catatonia, Obsessive-Compulsions \& Disorders of Movement (University Press, Cambridge, 2011).

Online Version

[16] A. Latteri, P. Arena, and P. Mazzone, "Characterizing deep brain stimulation effects in computationally efficient neural network models." Nonlinear Biomed. Phys. 5, 1-14 (2011).

Online Version

[17] L. J. Harris and J. B. Almerigi, "Probing the human brain with stimulating electrodes: The story of Roberts Bartholow's (1874) experiment on Mary Rafferty.” Brain Cogn. 70, 92-115 (2009).

Online Version

[18] P. R. Schuurman, D. A. Bosch, P. M. Bossuyt, G. J. Bonsel, E. J. W. van Someren, R. M. A. de Bie, M. P. Merkus, and J. D. Speelman, "A comparison of continuous thalamic stimulation and thalamotomy for suppression of severe tremor." N. Engl. J. Med. 342, 461-468 (2000).

Online Version 
[19] M. I. Hariz, P. Blomstedt, and L. Zrinzo, "Deep brain stimulation between 1947 and 1987: The untold story.” Neurosurg. Focus 29, 10106 (2010).

Online Version

[20] A. L. Benabid, P. Pollak, A. Louveau, S. Henry, and J. de Rougemont, "Combined (thalamotomy and stimulation) stereotactic surgery of the VIM thalamic nucleus for bilateral Parkinson disease.” Appl. Neurophysiol. 50, 344-346 (1987).

Online Version

[21] Mayfield Clinic, University of Cincinnati Department of Neurosurgery, Ohio, “Deep brain stimulation for movement disorders." Mayfield Clinic \& Spine Institute, http://www.mayfieldclinic.com/PDF/PE-DBS.pdf (2013).

[22] I. Osorio, M. G. Frei, S. Sunderam, J. Giftakis, N. C. Bhavaraju, S. F. Schaffner, and S. B. Wilkinson, "Automated seizure abatement in humans using electrical stimulation." Ann. Neurol. 57, 258-268 (2005).

Online Version

[23] P. Limousin, P. Pollak, A. Benazzouz, D. Hoffmann, J. F. Le Bas, E. Broussolle, J. E. Perret, and A. L. Benabid, "Effect of Parkinsonian signs and symptoms of bilateral subthalamic nucleus stimulation.” Lancet 345, 91-95 (1995).

Online Version

[24] R. Kumar, A. M. Lozano, Y. J. Kim, W. D. Hutchison, E. Sime, E. Halket, and A. E. Lang, "Double-blind evaluation of subthalamic nucleus deep brain stimulation in advanced Parkinson's disease.” Neurology 51, 850-855 (1998).

Online Version 
[25] A. Benazzouz, C. Gross, J. Féger, T. Boraud, and B. Bioulac, "Reversal of rigidity and improvement in motor performance by subthalamic high-frequency stimulation in MPTP-treated Monkeys.” Eur. J. Neurosci. 5, 382-389 (1993). Online Version

[26] A. Benazzouz, D. M. Gao, Z. G. Ni, B. Piallat, R. Bouali-Benazzouz, and A. L. Benabid, "Effect of high-frequency stimulation of the subthalamic nucleus on the neuronal activities of the substantia nigra pars reticulata and ventrolateral nucleus of the thalamus in the rat." Neuroscience 99, 289-295 (2000).

Online Version

[27] MDR Regulation and FDA publications, "Recently-Approved Devices - Activa® Parkinson's Control System P960009/S7." Department of Health \& Human Services, http://www.fda.gov/MedicalDevices/ProductsandMedicalProcedures/DeviceApprovals andClearances/Recently-ApprovedDevices/ucm083894.htm (Jan-14-2002).

[28] E. B. Montgomery and K. B. Baker, "Mechanisms of deep brain stimulation and future technical developments.” Neurol. Res. 22, 259-266 (2000).

Online Version

[29] J. E. Rubin and D. Terman, "High frequency stimulation of the subthalamic nucleus eliminates pathological thalamic rhythmicity in a computational model.” J. Comput. Neurosci. 16, 211-235 (2004).

Online Version

[30] C. J. Wilson, B. Beverlin, and T. Netoff, "Chaotic desynchronization as the therapeutic mechanism of deep brain stimulation.” Front. Syst. Neurosci. 5, 00050 (2011).

Online Version 
[31] I. R. Epstein and J. A. Pojman, An introduction to nonlinear chemical dynamics: Oscillations, waves, patterns, and chaos (Oxford University Press, New York, 1998).

[32] A.T. Winfree, "The prehistory of the Belousov-Zhabotinsky oscillator." J. Chem. Educ. 61, 661 (1984).

Online Version

[33] S. A. Oprisan, "A geometric approach to phase resetting estimation based on mapping temporal to geometric phase." In Phase Response Curves in Neuroscience, edited by N. W. Schultheiss, A. A. Prinz, and R. J. Butera (Springer, New York, 2012), pp. 131162.

Online Version

[34] J. J. Hopfield, "Neurons, dynamics and computation.” Phys. Today 47, 40-46 (2008). Online Version

[35] R. J. Field and R. M. Noyes, "Oscillations in chemical systems. IV. Limit cycle behavior in a model of a real chemical reaction.” J. Chem. Phys. 60, 1877-1884 (1974). Online Version

[36] A. M. Zhabotinsky, F. Buchholtz, A. B. Kiyatkin, and I. R. Epstein, "Oscillations and waves in metal-ion-catalyzed bromate oscillating reactions in highly oxidized states.” J. Phys. Chem. 97, 7578-7584 (1993).

Online Version

[37] H. Skødt and P. G. Sørensen, "Antispirals in an artificial tissue of oscillatory cells." Phys. Rev. E 68, 020902 (2003).

Online Version 
[38] O.-U. Kheowan, E. Mihaliuk, B. Blasius, I. Sendiña-Nadal, and K. Showalter, "Wave mediated synchronization of nonuniform oscillatory media." Phys. Rev. Lett. 98, 074101 (2007).

Online Version

[39] M. Toiya, H. O. González-Ochoa, V. K. Vanag, S. Fraden, and I. R. Epstein, "Synchronization of chemical micro-oscillators.” J. Phys. Chem. Lett. 1, 1241-1246 (2010).

Online Version

[40] J. Maselko, J. S. Reckley, and K. Showalter, "Regular and irregular spatial patterns in an immobilized-catalyst Belousov-Zhabotinsky reaction.” J. Phys. Chem. 93, 27742780 (1989).

Online Version

[41] K. Yoshikawa, R. Aihara, and K. Agladze, "Size-dependent Belousov-Zhabotinsky oscillation in small beads.” J. Phys. Chem. A 102, 7649-7652 (1998).

Online Version

[42] A. F. Taylor, M. R. Tinsley, F. Wang, Z. Huang, and K. Showalter, "Dynamical quorum sensing and synchronization in large populations of chemical oscillators." Science 323, 614-617 (2009).

Online Version

[43] H. Fukuda, N. Tamari, H. Morimura, and S. Kai, "Entrainment in a chemical oscillator chain with a pacemaker.” J. Phys. Chem. A 109, 11250-11254 (2005).

Online Version 
[44] R. Yoshida, E. Kokufuta, and T. Yamaguchi, "Beating polymer gels coupled with a nonlinear chemical reaction.” Chaos 9, 260-266 (1999).

Online Version

[45] X.-J. Lu, L. Ren, Q.-Y. Gao, Y.-Y. Yang, Y.-M. Zhao, J. Huang, X.-L. Lv, and I. R. Epstein, "Multiple length scale instabilities of unidirectional pulse propagation in a diffusion-fed gel.” J. Phys. Chem. Lett. 4, 3891-3896 (2013). Online Version

[46] M. Toiya, V. K. Vanag, and I. R. Epstein, "Diffusively coupled chemical oscillators in a microfluidic assembly.” Angew. Chem. Int. Ed. Engl. 120, 7867-7869 (2008).

Online Version

[47] N. Tompkins, N. Li, C. Girabawe, M. Heymann, G. B. Ermentrout, I. R. Epstein, and S. Fraden, "Testing Turing's theory of morphogenesis in chemical cells." PNAS 111, 4397-4402 (2014).

Online Version

[48] M. R. Tinsley, A. F. Taylor, Z. Huang, and K. Showalter, "Complex organizing centers in groups of oscillatory particles.” Phys. Chem. Chem. Phys. 13, 17802-17808 (2011). Online Version

[49] N. Nishiyama and K. Eto, "Experimental study on three chemical oscillators coupled with time delay.” J. Chem. Phys. 100, 6977-6978 (1994).

Online Version

[50] M. R. Tinsley, S. Nkomo, and K. Showalter, "Chimera and phase-cluster states in populations of coupled chemical oscillators." Nat. Phys. 8, 662-665 (2012).

Online Version 
[51] S. H. Strogatz, "From Kuramoto to Crawford: Exploring the onset of synchronization in populations of coupled oscillators.” Phys. D 143, 1-20 (2000).

Online Version

[52] V. K. Vanag, L. Yang, M. Dolnik, A. M. Zhabotinsky, and I. R. Epstein, "Oscillatory cluster patterns in a homogeneous chemical system with global feedback." Nature 406, 389-391 (2000).

Online Version

[53] A. Weber, Y. Prokazov, W. Zuschratter, and M. J. B. Hauser, "Desynchronization of glycolytic oscillations in yeast cell populations." PLoS ONE 7, e43276 (2012). Online Version

[54] A. F. Taylor, M. R. Tinsley, F. Wang, and K. Showalter, "Phase clusters in large populations of chemical oscillators.” Angew. Chem. Int. Ed. 50, 10161-10164 (2011).

Online Version

[55] L. M. Pecora, F. Sorrentino, A. M. Hagerstrom, T. E. Murphy, and R. Roy, "Cluster synchronization and isolated desynchronization in complex networks with symmetries."

Nat. Commun. 5, 4079 (2014).

Online Version

[56] K. P. Wright, A. W. McHill, B. R. Birks, B. R. Griffin, T. Rusterholz, and E. D. Chinoy, "Entrainment of the human circadian clock to the natural light-dark cycle." Curr. Biol. 23, 1554-1558 (2013).

Online Version

[57] S. Panda, J. B. Hogenesch, and S. A. Kay, "Circadian rhythms from flies to human." Nature 417, 329-335 (2002). 
Online Version

[58] S. Coombes and P. Bressloff, "Mode locking and Arnold tongues in integrate-and-fire neural oscillators.” Phys. Rev. E 60, 2086-2096 (1999).

Online Version

[59] V. Petrov, Q. Ouyang, and H. L. Swinney, "Resonant pattern formation in a chemical system.” Nature 388, 655-657 (1997).

Online Version

[60] A. Lin, A. Hagberg, E. Meron, and H. Swinney, "Resonance tongues and patterns in periodically forced reaction-diffusion systems." Phys. Rev. E 69, 066217 (2004). Online Version

[61] J. L. Atlee and A. D. Bernstein, "Cardiac rhythm management devices (part I): Indications, device selection, and function.” Anesthesiology 95, 1265-1280 (2001). Online Version

[62] A. G. Rapsang and P. Bhattacharyya, "Pacemakers and implantable cardioverter defibrillators - general and anesthetic considerations.” Braz. J. Anesthesiol. 64, 205214 (2014).

Online Version

[63] C. Graves, L. Glass, D. Laporta, R. Meloche, and A. Grassino, "Respiratory phase locking during mechanical ventilation in anesthetized human subjects.” Am. J. Physiol. 250, R902-909 (1986).

Online Version

[64] J. Wang, S. Kádár, P. Jung, and K. Showalter, "Noise driven avalanche behavior in subexcitable Media.” Phys. Rev. Lett. 82, 855-858 (1999). 


\section{Online Version}

[65] S. Kádár, J. Wang, and K. Showalter, "Noise-supported travelling waves in subexcitable media." Nature 391, 770-772 (1998).

Online Version

[66] P. Jung, A. Cornell-Bell, F. Moss, S. Kádár, J. Wang, and K. Showalter, "Noise sustained waves in subexcitable media: From chemical waves to brain waves." Chaos 8, 567-575 (1998).

Online Version

[67] C. Zhou, J. Kurths, I. Kiss, and J. Hudson, "Noise-enhanced phase synchronization of chaotic oscillators.” Phys. Rev. Lett. 89, 014101 (2002).

Online Version

[68] I. Z. Kiss, Y. Zhai, J. L. Hudson, C. Zhou, and J. Kurths, "Noise enhanced phase synchronization and coherence resonance in sets of chaotic oscillators with weak global coupling." Chaos 13, 267-278 (2003).

Online Version

[69] A. Neiman and D. Russell, "Synchronization of noise-induced bursts in noncoupled sensory neurons.” Phys. Rev. Lett. 88, 138103 (2002).

Online Version 


\section{Chapter 2}

\section{Photosensitive BZ Chemical Oscillators}

\subsection{Introduction}

The photosensitive modification of the $\mathrm{BZ}$ reaction, where the tris(2,2-bipyridine) ruthenium complex, $\mathrm{Ru}(\mathrm{bpy})_{3}^{2+}$, is used as a catalyst, was first reported by Demas and Diemente [1] in 1973. The ruthenium complex was used as a luminescence indicator, where the reaction mixture was irradiated with UV light to visualize the oscillatory behavior. In 1983, the ruthenium-catalyzed $\mathrm{BZ}$ reaction was shown to be sensitive to irradiation by visible light, with $\lambda$ $=445 \mathrm{~nm}$ [2]. Illuminated ruthenium-catalyzed BZ systems have since been used to demonstrate variation of spatial and temporal behaviors, such as the triggering of diffusion phase waves [3], annihilation [4, 5], initiation [6, 7], controlling waves [8-11], and suppression or activation of oscillations [2]. In the present study, a photosensitive BZ system was used to allow for monitoring of oscillatory particles, with a certain coupling topology, and for external forcing with photochemical feedback imposed through illumination by visible light, with $\lambda=440-$ $460 \mathrm{~nm}$. Moreover, for uncoupled oscillators, the characteristic features of individual oscillators' response to perturbations was measured to construct the phase response curve (PRC).

\subsubsection{The BZ Mechanism}

The $\mathrm{BZ}$ reaction can be described as the oxidation of an organic reagent (e.g., malonic acid, $\mathrm{MA} \equiv \mathrm{CH}_{2}(\mathrm{COOH})_{2}$ ) by bromate, $\mathrm{BrO}_{3}^{-}$, in an acidic solution (e.g., $\mathrm{H}_{2} \mathrm{SO}_{4}$ ) in the 
presence of a metal complex catalyst (e.g., $\mathrm{Ru}(\mathrm{bpy})_{3}^{2+}$ ), which is given by the following overall reaction [12]:

$$
2 \mathrm{BrO}_{3}^{-}(\mathrm{aq})+3 \mathrm{MA}(\mathrm{aq})+2 \mathrm{H}^{+}(\mathrm{aq}) \rightarrow 2 \mathrm{BrMA}(\mathrm{aq})+4 \mathrm{H}_{2} \mathrm{O}(\mathrm{l})+3 \mathrm{CO}_{2}(\mathrm{~g})
$$

This reaction proceeds by cyclic production of the intermediate species bromous acid, $\mathrm{HBrO}_{2}$, and bromide ions, $\mathrm{Br}^{-}$. It involves a complex mechanism that was described by a ten-step basic mechanism proposed by Noyes et al. termed the FKN mechanism [13]. The Oregonator model, developed by Field and Noyes in 1974, reduced the FKN mechanism to five steps, which reflects the essential features of BZ dynamics [14]. Recently, the ZBKE mechanism, as shown in Table 2.1, was proposed for effective simulations of experimental observations [15]. The species $\mathrm{HBrO}_{2}^{+}$is considered to play an active part in the ZBKE model. The abbreviations $\mathrm{cat}_{\text {red }}$ and cat $_{\text {ox }}$ represent the reduced and oxidized states of the metal complex catalysts, respectively.

Table 2.1: The Detailed Zhabotinsky, Buchholtz, Kiyatkin, and Epstein (ZBKE) Mechanism of the Belousov-Zhabotinsky (BZ) Reaction [15].

\begin{tabular}{|l|c|}
\hline Reaction & Reaction number \\
$\mathrm{H}^{+}+\mathrm{Br}^{-}+\mathrm{HOBr} \rightleftarrows \mathrm{Br}_{2}+\mathrm{H}_{2} \mathrm{O}$ & $\mathrm{ZBKE} /(\mathrm{R} 1)$ \\
$\mathrm{H}^{+}+\mathrm{BrrO}_{2} \rightleftarrows 2 \mathrm{HOBr}$ & $\mathrm{ZBKE} /(\mathrm{R} 2)$ \\
$2 \mathrm{HBrO}_{2} \rightleftarrows \mathrm{HBrO}_{3} \rightleftarrows \mathrm{HBrO} \mathrm{O}_{2}+\mathrm{HOBr}$ & $\mathrm{ZBKE} /(\mathrm{R} 3)$ \\
$\mathrm{H}^{+}+\mathrm{HBrO}_{2} \rightleftarrows \mathrm{H}_{2} \mathrm{BrO}_{2}^{+}$ & $\mathrm{ZBKE} /(\mathrm{R} 4 \mathrm{a})$ \\
$\mathrm{HBrO}_{2}+\mathrm{H}_{2} \mathrm{BrO}_{2}^{+} \rightleftarrows \mathrm{HBrO}_{3}+\mathrm{HOBr}^{+} \mathrm{H}^{+}$ & $\mathrm{ZBKE} /(\mathrm{R} 4 \mathrm{~b})$ \\
$\mathrm{H}^{+}+\mathrm{BrO}_{3}^{-} \rightleftarrows \mathrm{HBrO}_{3}$ & $\mathrm{ZBKE} /(\mathrm{R} 4 \mathrm{c})$ \\
$\mathrm{H}^{+}+\mathrm{HBrO}_{3}+\mathrm{HBrO}_{2} \rightleftarrows \mathrm{HBrO}_{2}^{+}+\mathrm{BrO}_{2}^{+}+\mathrm{H}_{2} \mathrm{O}$ & $\mathrm{ZBKE} /(\mathrm{R} 5 \mathrm{a})$ \\
\hline
\end{tabular}




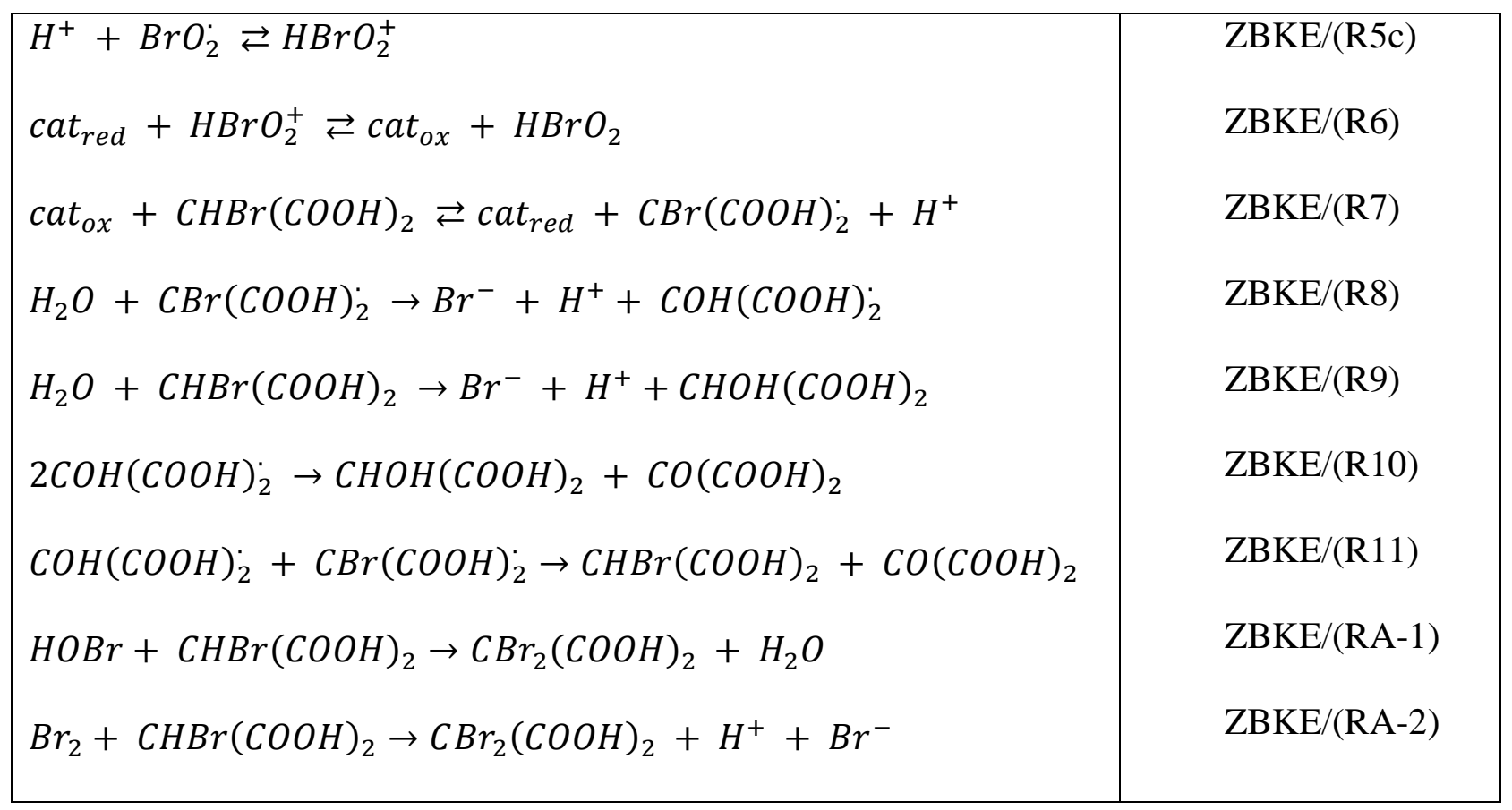

A simplification of the $\mathrm{BZ}$ mechanism into three primary processes, $\mathrm{A}, \mathrm{B}$, and $\mathrm{C}$, has been suggested to capture the essence of the $\mathrm{BZ}$ reaction to allow the construction of mathematical models [15]. In process A, removing the inhibitor species of the reaction, $\mathrm{Br}^{-}$, takes place. In process $\mathrm{B}$, autocatalytic production of the activator species of the reaction, $\mathrm{HBrO}_{2}$, takes place while oxidizing the catalyst. More of the inhibitory species, $\mathrm{Br}^{-}$, is then formed by reducing the catalyst in process $\mathrm{C}$, which suppresses process B and restarts process A. The key equations summarizing processes $\mathrm{A}, \mathrm{B}$, and $\mathrm{C}$ in the ZBKE scheme are described as follows [16].

Process (A): Removal of the inhibitor, $B r^{-}$.

$$
\begin{aligned}
& \text { (A1) } \mathrm{HBrO}_{3}+\mathrm{Br}^{-}+\mathrm{H}^{+} \rightleftarrows \mathrm{HBrO}_{2}+\mathrm{HOBr} \\
& \text { (A2) } \mathrm{HBrO}_{2}+\mathrm{Br}^{-}+\mathrm{H}^{+} \rightleftarrows 2 \mathrm{HOBr}
\end{aligned}
$$

(A) $\mathrm{HBrO}_{3}+2 \mathrm{Br}^{-}+2 \mathrm{H}^{+} \rightleftarrows 3 \mathrm{HOBr}$

Process (B): Autocatalytic production of the activator, $\mathrm{HBrO}_{2}$. 
(B1) $\mathrm{HBrO}_{3}+\mathrm{HBrO}_{2}+2 \mathrm{H}^{+} \rightleftarrows 2 \mathrm{HBrO}_{2}^{+}+\mathrm{H}_{2} \mathrm{O}$
(B2) cat $\left._{\text {red }}+\mathrm{HBrO}_{2}^{+} \rightleftarrows \mathrm{cat}_{\mathrm{ox}}+\mathrm{HBrO}_{2}\right\} \times 2$

(B) $\mathrm{HBrO}_{2}+2 \mathrm{cat}_{\text {red }}+\mathrm{HBrO}_{3}+2 \mathrm{H}^{+} \rightarrow 2 \mathrm{HBrO}_{2}+2 \mathrm{cat}_{\mathrm{ox}}+\mathrm{H}_{2} \mathrm{O}$

where the autocatalytic production of the activator is limited by the following equation [12]:

(B3) $2 \mathrm{HBrO}_{2} \rightleftarrows \mathrm{HBrO}_{3}+\mathrm{HOBr}$

Process (C): Regeneration of the inhibitor, $\mathrm{Br}^{-}$.

(C1) $\mathrm{cat}_{\mathrm{ox}}+\mathrm{B} \rightleftarrows \mathrm{cat}_{\mathrm{red}}+\mathrm{B}^{\cdot}+\mathrm{H}^{+}$

$(\mathrm{C} 2) \mathrm{B}^{\cdot} \rightarrow \mathrm{fBr}^{-}+$products

$(\mathrm{C} 2) \mathrm{B} \rightarrow \mathrm{Br}^{-}$

(C) cat $_{\mathrm{ox}}+\mathrm{B} \rightleftarrows \mathrm{cat}_{\mathrm{red}}+\mathrm{fBr}^{-}+\mathrm{H}^{+}+$products

Here, $B$ is $(\mathrm{MA}+\mathrm{BrMA})$ and $\mathrm{f}$ is a stoichiometric factor that indicates the amount of production of bromide ions per one unit of the reduced catalyst or photochemical decomposition of oxidized organic species [17]. The BZ mechanism can be described via three processes. First, the consumption of the initial concentration of bromide ion, $\mathrm{Br}^{-}$, by reaction with bromate, $\mathrm{BrO}_{3}^{-}$, occurs in $\mathrm{A}$. In this process, the intermediate species bromous acid, $\mathrm{HBrO}_{2}$, is maintained at a low steady state concentration. In the next process, B, bromous acid is autocatalytically produced and the cycle of oscillations in the catalyst oxidation state is initiated (from orange, the reduced form, $\mathrm{Ru}(\mathrm{bpy})_{3}^{2+}$, to green, the oxidized form, $\left.\mathrm{Ru}(\mathrm{bpy})_{3}^{3+}\right)$. Here, the bromous acid concentration is several orders of magnitude greater than that in process $\mathrm{A}$, while the bromide ion reaches its lowest concentration. In the final process, $\mathrm{C}$, the malonic acid, $M A$, and 
bromomalonic acid, $\operatorname{BrMA}$, sustain the oscillation cycle by the production of the reduced form of the catalyst and the regeneration of $\mathrm{Br}^{-}$, until it becomes sufficient to inhibit the autocatalytic production of bromous acid. Thus, the process will switch from process $\mathrm{C}$ to process $\mathrm{A}$, initiating the cycle again $[13,18]$. The oscillation cycle repeats until the reaction reaches equilibrium, where all the malonic acid is depleted and is transformed into organic products and the free energy of the system is minimized [19].

This model is adjusted to incorporate light sensitivity in ruthenium-catalyzed BZ system. In the present study, a mechanism of positive feedback in process B is included to increase the autocatalytic production of $\mathrm{HBrO}_{2}$ in the above ZBKE mechanism. However, in a photoinhibitory scheme, a mechanism for negative feedback in process $\mathrm{C}$ is included. The primary step in the ruthenium-catalyzed $\mathrm{BZ}$ reaction is the absorption of light by $\mathrm{Ru}(\mathrm{bpy})_{3}^{2+}$ and the transition to its excited state, $\mathrm{Ru}(\mathrm{bpy})_{3}^{2+*}$, which is a strong reducing agent. The reduction potential of $\mathrm{Ru}(\mathrm{bpy})_{3}^{3+} / \mathrm{Ru}(\mathrm{bpy})_{3}^{2+*}$ is $-0.86 \mathrm{~V}$, while that for $\mathrm{Ru}(\mathrm{bpy})_{3}^{3+} / \mathrm{Ru}(\mathrm{bpy})_{3}^{2+}$ is $1.26 \mathrm{~V}$ [12]. Thus, the excited form of the catalyst reacts directly with bromate, $\mathrm{BrO}_{3}^{-}$, leading to the formation of the activator species, $\mathrm{HBrO}_{2}$. The mechanism accounting for photo-induction of the $\mathrm{Ru}(\mathrm{bpy})_{3}^{2+}-$ catalyzed reaction was proposed by Hanazaki et al. as follows [20]:

$$
\begin{aligned}
& \mathrm{Ru}(\text { bpy })_{3}^{2+*}+\mathrm{BrO}_{3}^{-}+2 \mathrm{H}^{+} \rightarrow \mathrm{BrO}_{2}^{-}+\mathrm{Ru}(\text { bpy })_{3}^{3+}+\mathrm{H}_{2} \mathrm{O} \\
& \mathrm{BrO}_{2}+\mathrm{Ru}(\mathrm{bpy})_{3}^{2+}+\mathrm{H}^{+} \rightarrow \mathrm{Ru}(\mathrm{bpy})_{3}^{3+}+\mathrm{HBrO}_{2}
\end{aligned}
$$

$$
\mathrm{Ru}(\text { bpy })_{3}^{2+*}+\mathrm{Ru}(\text { bpy })_{3}^{2+}+\mathrm{BrO}_{3}^{-}+3 \mathrm{H}^{+} \rightarrow \mathrm{HBrO}_{2}+2 \mathrm{Ru}(\text { bpy })_{3}^{3+}+\mathrm{H}_{2} \mathrm{O}
$$

This reaction will increase the production of activator species at the expense of production of inhibitor in the reaction, as illustrated by Eq. 2.3 in process (A). Thus, illumination will 
enhance the original autocatalytic net reaction, as given by Eq. 2.7 in process (B). The further reduction of excess of $\mathrm{HBrO}_{2}$ to bromide ions has also been suggested to occur as follows [20]:

$$
\mathrm{Ru}(\text { bpy })_{3}^{2+*}+3 \mathrm{Ru}(\text { bpy })_{3}^{2+}+\mathrm{HBrO}_{2}+3 \mathrm{H}^{+} \rightarrow \mathrm{Br}^{-}+4 \mathrm{Ru}(\text { bpy })_{3}^{3+}+2 \mathrm{H}_{2} \mathrm{O}
$$

\subsubsection{The ZBKE Model}

The ZBKE model reduces the full BZ mechanism, which involves many steps, into threevariable and two-variable mathematical models. The model allows frequency heterogeneity in the chemical oscillators, while the original Oregonator model does not permit significant variation in oscillator frequency $[21,22]$. The ZBKE model in Table 2.2 is written using the following definitions of reaction species $[14,15,16]: X=\left[\mathrm{HBrO}_{2}\right], Y=\left[\mathrm{Br}^{-}\right], Z=\left[\mathrm{cat}_{\text {ox }}\right], U=$ $\left[\mathrm{HBrO}_{2}^{+}\right], \mathrm{A}=\left[\mathrm{HBrO}_{3}\right]=h_{0}\left[\mathrm{NaBrO}_{3}\right]_{0} /\left(0.2+h_{0}\right)$, where $h_{0}$ is the Hammet acidity function, $H=h_{0}, \quad K_{\mathrm{HBrO}_{3}}=0.2+h_{0}, \quad B=\left[\mathrm{CHBr}(\mathrm{COOH})_{2}\right], \quad B^{\cdot}=\left[\mathrm{CBr}(\mathrm{COOH})_{2} \cdot\right], \quad C=\left(\left[\mathrm{cat}_{\text {ox }}\right]+\right.$ [cat $\left.\left.{ }_{r e d}\right]\right), P$ and $Q$ are $\mathrm{HOBr}$ and organic products, respectively, that do not affect the dynamics, while $q$ is an adjustable stoichiometric factor that can be varied from 0.5 to 1.0 for the oscillatory regime [16].

Table 2.2: The Zhabotinsky, Buchholtz, Kiyatkin, and Epstein Model.

$$
\begin{gathered}
X+Y+H \stackrel{k_{2}}{\rightarrow} 2 P \\
\mathrm{~A}+\mathrm{Y}+H \stackrel{\mathrm{k}_{3}}{\rightarrow} \mathrm{X}+\mathrm{P} \\
2 X \stackrel{k_{4}}{\rightarrow} P+A \\
A+X+H \stackrel{k_{5}}{\rightleftharpoons} 2 \mathrm{U} \\
k_{-5}
\end{gathered}
$$




\begin{tabular}{|c|c|}
\hline $\begin{array}{c}k_{6} \\
k_{-6}\end{array}$ & $(2.21)$ \\
\hline$Z+\underset{k_{-7}}{\stackrel{k_{7}}{\rightleftharpoons}}(C-Z)+B+H$ & $(2.22)$ \\
\hline$B \stackrel{k_{8}}{\rightarrow} q Y+Q$ & $(2.23)$ \\
\hline$B \stackrel{k_{9}}{\rightarrow} Y$ & (2.24) \\
\hline
\end{tabular}

By assuming the concentrations of $\mathrm{A}, \mathrm{B}, \mathrm{C}$, and $\mathrm{H}$ as constant and treating the intermediate species $B^{\prime}$ and $\mathrm{U}$ with a steady-state approximation, the overall ZBKE differential equations are reduced to the three variable differential equation, with the following dimensionless form [24]:

$$
\begin{aligned}
& \frac{d X}{d t}=-k_{2} h_{0} X Y+k_{3} h_{0} A Y-2 k_{4} X^{2}-k_{5} h_{0} A X+k_{-5} U_{s S}^{2}+k_{6} U_{s s}(C-Z)-k_{-6} X Z \\
& \frac{d Y}{d t}=-k_{2} h_{0} X Y-k_{3} h_{0} A X+q\left[\frac{k_{7} k_{8} B Z}{k_{8}+k_{-7} h_{0}(C-Z)}\right]+k_{9} B \\
& \frac{d Z}{d t}=k_{6} U_{s s}(C-Z)-k_{-6} X Z-\left[\frac{k_{7} k_{8} B Z}{k_{8}+k_{-7} h_{0}(C-Z)}\right]
\end{aligned}
$$

where the value of $U$ is at the steady state concentration.

$$
\mathrm{U}_{\mathrm{ss}}=\frac{1}{4 \mathrm{k}_{-5}}\left(\mathrm{k}_{6}(\mathrm{C}-\mathrm{Z})+\left[\mathrm{k}_{6}^{2}(\mathrm{C}-\mathrm{Z})^{2}+16 \mathrm{k}_{5} \mathrm{k}_{-5} \mathrm{~h}_{0} \mathrm{Ax}+8 \mathrm{k}_{-5} \mathrm{k}_{-6} \mathrm{xz}\right]^{1 / 2}\right)
$$

The dimensionless ZBKE equations after scaling, as shown in Table 2.3, are given by

$$
\begin{aligned}
& \frac{d x}{d \tau}=\frac{1}{\varepsilon_{1}}\left(-x^{2}-x+\varepsilon_{2} \gamma u_{s s}^{2}+u_{s s}(1-z)+\delta x z+\mu y-x y\right) \\
& \frac{d y}{d \tau}=\frac{1}{\varepsilon_{4}}\left(-x y-\mu y+q\left[\frac{\alpha z}{\left(\varepsilon_{3}+1-z\right)}+\beta\right]\right) \\
& \frac{d z}{d \tau}=u_{s s}(1-z)-\delta x z-\left[\frac{\alpha z}{\left(\varepsilon_{3}+1-z\right)}\right]
\end{aligned}
$$


Table 2.3: Scaling Parameters for the Dimensionless Zhabotinsky, Buchholtz, Kiyatkin, and Epstein Model [24].

\begin{tabular}{|c|c|}
\hline Variable & Scaling \\
\hline$X$ & $k_{5} h_{0} A x / 2 k_{4}$ \\
\hline$Y$ & $k_{5} A y / k_{2}$ \\
\hline$Z$ & $C z$ \\
\hline$U$ & $\left(k_{5} h_{0} A\right) 2 u / 2 k_{4} k_{6} C$ \\
\hline$t$ & $2 k_{4} C z /\left(k_{5} h_{0} A\right)^{2}$ \\
\hline Parameter & Scaling \\
\hline$\varepsilon_{1}$ & $k_{5} h_{0} A / 2 k_{4} C$ \\
\hline$\varepsilon_{2}$ & $\left(k_{5} h_{0} A\right)^{2} / 2 k_{4} k_{6} C$ \\
\hline$\varepsilon_{3}$ & $k_{8} / k_{-7} h_{0} C$ \\
\hline$\varepsilon_{4}$ & $k_{5} A / k_{2} C$ \\
\hline$\alpha$ & $2 k_{4} k_{7} k_{8} B / k_{5}^{2} k_{-7} h_{0}^{3} A^{2}$ \\
\hline$\beta$ & $2 k_{4} k_{9} B /\left(k_{5} h_{0} A\right)^{2}$ \\
\hline$\mu$ & $2 k_{3} k_{4} / k_{2} k_{5} h_{0}$ \\
\hline$\gamma$ & $k_{-5} / k_{6}$ \\
\hline$\delta$ & $k_{-6} C / k_{5} h_{0} A$ \\
\hline
\end{tabular}

For an excitatory system, the $\mathrm{HBrO}_{2}$ species contributes to the observed behavior at a lower concentration of $M A$ and $\mathrm{Br}^{-}$in the recipe, while increasing the concentration of the acid and bromate reactants. In this case, the inhibitor species bromide ion $[Y]$ can be treated with the 
steady state approximation, and the ZBKE model can be reduced to a two-variable system as follows [15]:

$$
\begin{aligned}
& \frac{d X}{d t}=\left[\frac{-k_{2} X+k_{3} A}{k_{2} X+k_{3} A}\right]\left(q\left[\frac{k_{7} k_{8} B Z}{k_{8}+k_{-7} h_{0}(C-Z)}\right]+k_{9} B\right)-2 k_{4} X^{2}-k_{5} h_{0} A X+k_{-5} U_{S S}^{2}+ \\
& k_{6} U_{S S}(C-Z)-k_{-6} X Z \\
& \frac{d Z}{d t}=k_{6} U_{S S}(C-Z)-k_{-6} X Z-\left[\frac{k_{7} k_{8} B Z}{k_{8}+k_{-7} h_{0}(C-Z)}\right]
\end{aligned}
$$

A simulation of the BZ reaction with the two-variable model is shown in Figure 2.1.

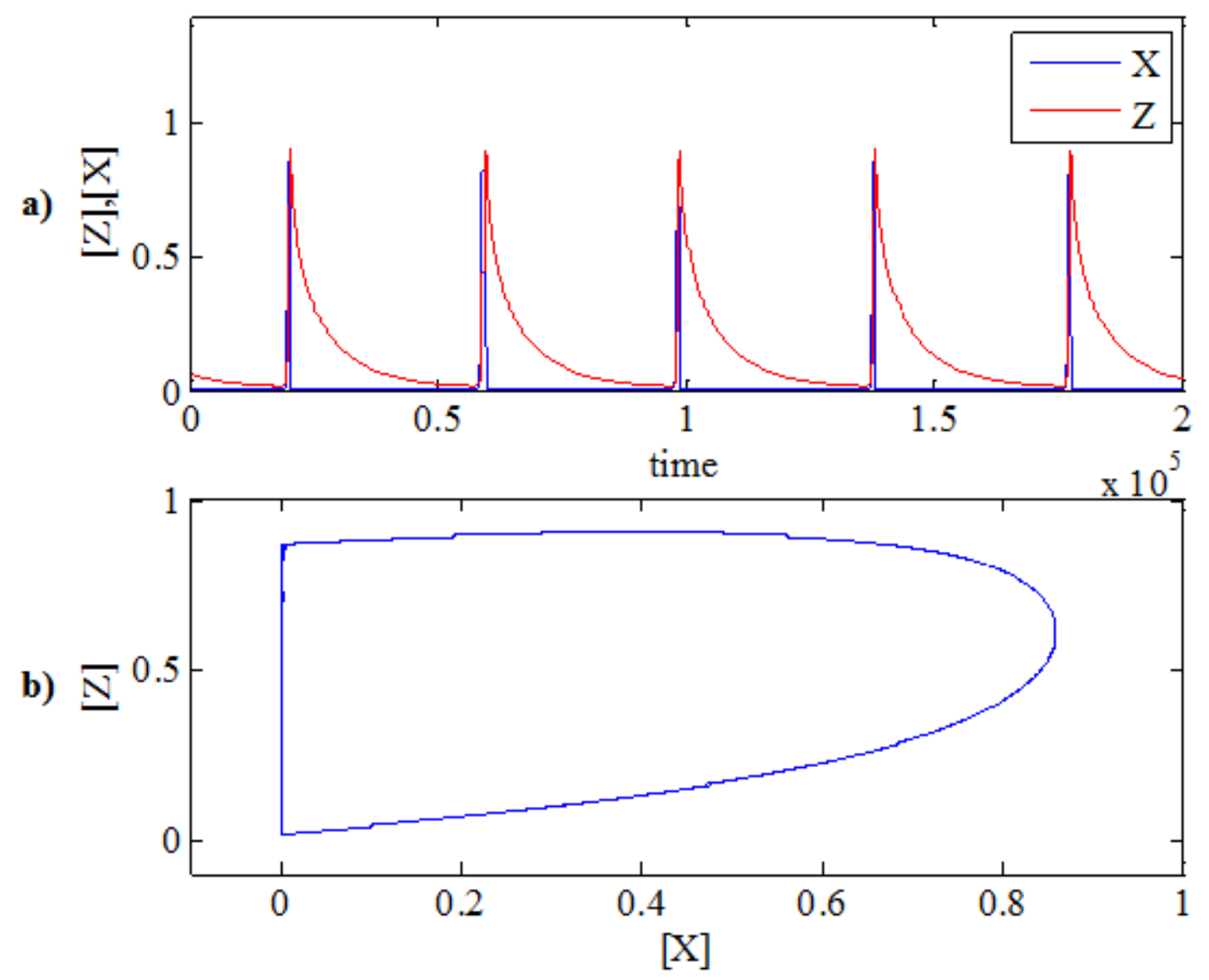

FIG. 2.1: Simulation of the Belousov-Zhabotinsky (BZ) reaction with the ZBKE model. a) The oscillation in bromous acid, $X$ (blue), and the catalyst, $Z$ (red). b) A plot of $X$ vs. $Z$ showing the limit cycle of the $\mathrm{BZ}$ reaction. 


\subsubsection{The Modified ZBKE Model for the Photosensitive BZ System}

In 1990 Krug et al. [17] proposed a simple modified Oregonator model for the photosensitive BZ reaction. The model accounts for the inhibiting effects of illumination that accompanies the production of bromide from bromomalonic acid, which in turn inhibits the autocatalytic production of bromous acid. This model was further modified in 1997 by Kadar $e t$ al. to include the photochemical production of bromous acid from bromate ion at high intensity illumination [25]. In the present study, we used the photochemical excitatory effects of the ruthenium-catalyzed $\mathrm{BZ}$ system, where illumination promotes the oxidation of $\mathrm{Ru}(\mathrm{bpy})_{3}^{2+}$, which accelerates the autocatalytic reaction. The key equation for the photo-excitatory ZBKE model to account for generation of activator, $X$, from the reaction of $\mathrm{Ru}(\mathrm{bpy})_{3}^{2+*}, E$, with $\mathrm{BrO}_{3}^{-}$, $A$, is as follows [25]:

$$
E+A \rightarrow X+2 Z
$$

while a competitive reaction accounts for generation of inhibitor, $Y$, from the reaction of $\mathrm{Ru}(\mathrm{bpy})_{3}^{2+*}, E$, with BrMA, $B$, is as follows [25]:

$$
E+B \rightarrow Y+Z
$$

Krug et al. [17] and then Vanag et al. [27] incorporated a term $\varphi$ to account for the production of bromide ions in a photo-inhibition scheme by illumination. Addition of the photoproduction term to the non-dimensional two-variable ZBKE model using the scaling parameters for $\mathrm{HBrO}_{2}, x$, and $\mathrm{Ru}(\mathrm{bpy})_{3}^{3+}, z$, is described as follows [26]:

$$
\begin{aligned}
& d x / d \tau=\frac{1}{\varepsilon_{1}}\left(\varphi-x^{2}-x+\varepsilon_{2} \gamma u_{s s}^{2}+u_{s s}(1-z)+\left[\frac{(\mu-x)}{(\mu+x)}\right]\left[\frac{q \alpha z}{\left(\varepsilon_{3}+1-z\right)}+\beta\right]\right) \\
& d z / d \tau=\varphi+u_{s s}(1-z)-\left[\frac{\alpha z}{\left(\varepsilon_{3}+1-z\right)}\right]
\end{aligned}
$$


Figure 2.2 shows a simulation of a photosensitive BZ reaction compared with a BZ reaction simulation using the two-variable equations and the appropriate values of the scaled parameters, as shown in Table 2.4. A photosensitive BZ system exhibits a reduction in the period of oscillation.
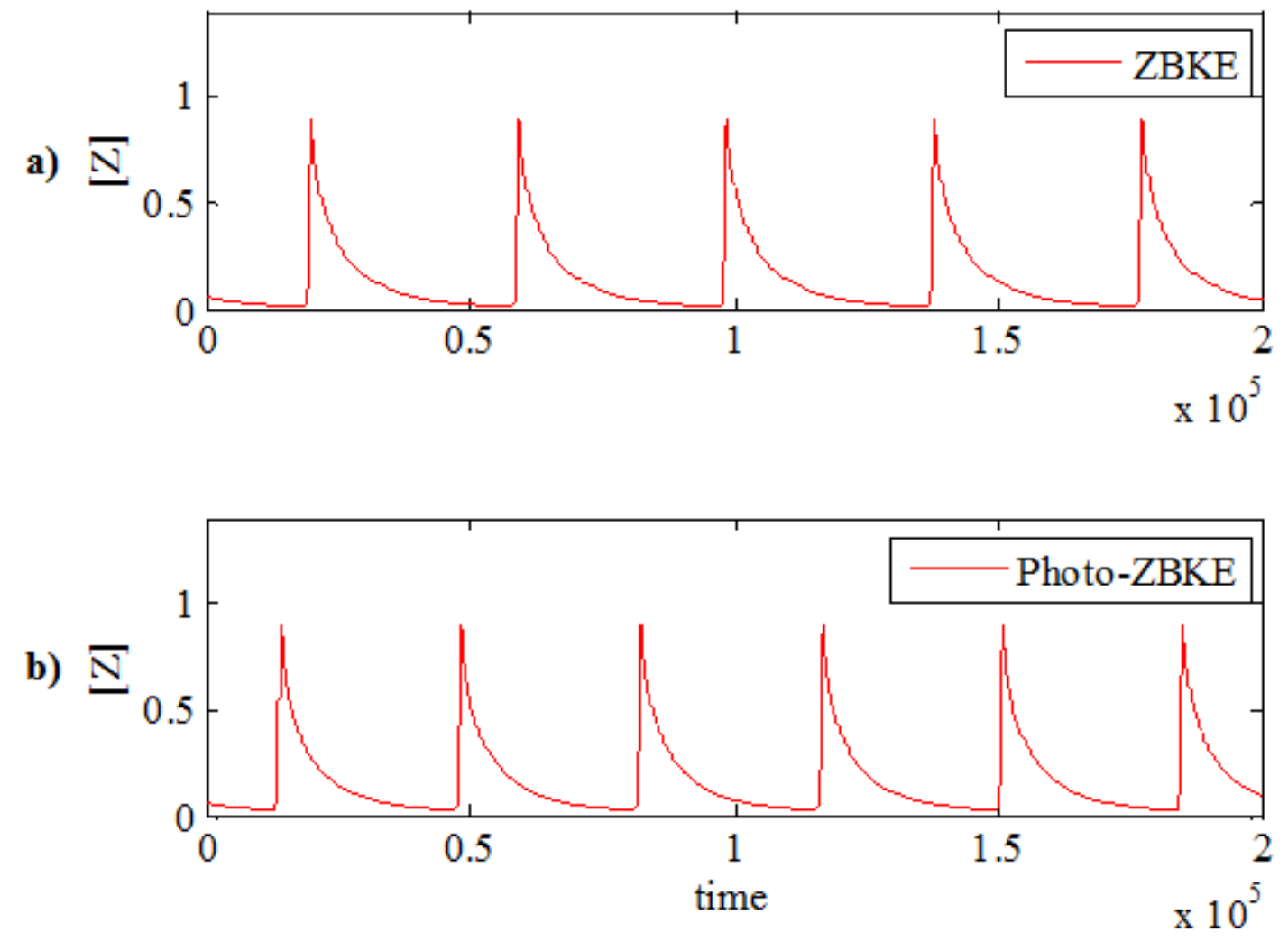

FIG. 2.2: Zhabotinsky, Buchholtz, Kiyatkin, and Epstein (ZBKE) and photo-ZBKE simulations. a) Time series showing oscillation in the catalyst, $Z$, with a longer oscillatory period, $3.93 \times 10^{5}$, in the typical Belousov-Zhabotinsky (BZ) system. b) Time series showing oscillation in the catalyst, $Z$, with a shorter oscillatory period, $3.43 \times 10^{5}$, in the photosensitive BZ system. 
Table 2.4: Parameter Values for the Two-Variable Zhabotinsky, Buchholtz, Kiyatkin, and Epstein Simulation [26].

\begin{tabular}{|c|c|c|c|}
\hline Parameter & Value & Parameter & Value \\
\hline$\varepsilon_{1}$ & 0.11 & $\beta$ & $1.7 \times 10^{-5}$ \\
$\varepsilon_{2}$ & $1.7 \times 10^{-5}$ & $\gamma$ & 1.2 \\
$\varepsilon_{3}$ & 0.016 & $\mu$ & $2.4 \times 10^{-4}$ \\
$\alpha$ & 0.1 & $q$ & 0.8 \\
\hline
\end{tabular}

\subsection{Phase Response Curve with Positive and Negative}

\section{Perturbations}

A stable limit cycle oscillator recovers to its standard natural frequency $(f)$, the number of oscillations per time unit, after an external perturbation causes a transient change of the firing rate or the oscillation period [28]. The oscillation period $(T)$ is the time required to complete one cycle of oscillation, where $f=1 / T$. The changes due to perturbation can be measured quantitatively by a phase response curve (PRC) [28], a graphical representation of an oscillators' response to systematic perturbations at different times of a cyclic oscillation (i.e., phases). Thus, the PRC for phase shifting experiments describes the magnitude of phase shifts made by perturbations at different phases of the cycle. Oscillations can be represented on a time or phase scale via the relation $t=\frac{\theta}{2 \pi} \times T_{0}$, as illustrated in Figure 2.3. For the phase $\theta$, the perturbation applied is calculated as the elapsed time from the most recent spiking time of the oscillator $\left(t_{\text {peak }}\right)$ to the stimulus time $\left(t_{s}\right)$ divided by the unperturbed period $\left(T_{0}\right)$, as described by Eq. 2.38 . For simplicity, $\theta=0$ is set at the spiking event of an oscillation, and the phase can take any 
value between 0 and $2 \pi$. The phase shift $(\delta \theta)$ is a measure of the change in a period with respect to its prior unperturbed period divided by the time of the unperturbed period, as described by Eq. 2.39 and shown in Figure 2.4.

$$
\begin{aligned}
& \theta=2 \pi\left(t_{s}-t_{\text {peak }}\right) / T_{0} \\
& \delta \theta=2 \pi\left(T_{0}-T_{1}\right) / T_{0}
\end{aligned}
$$

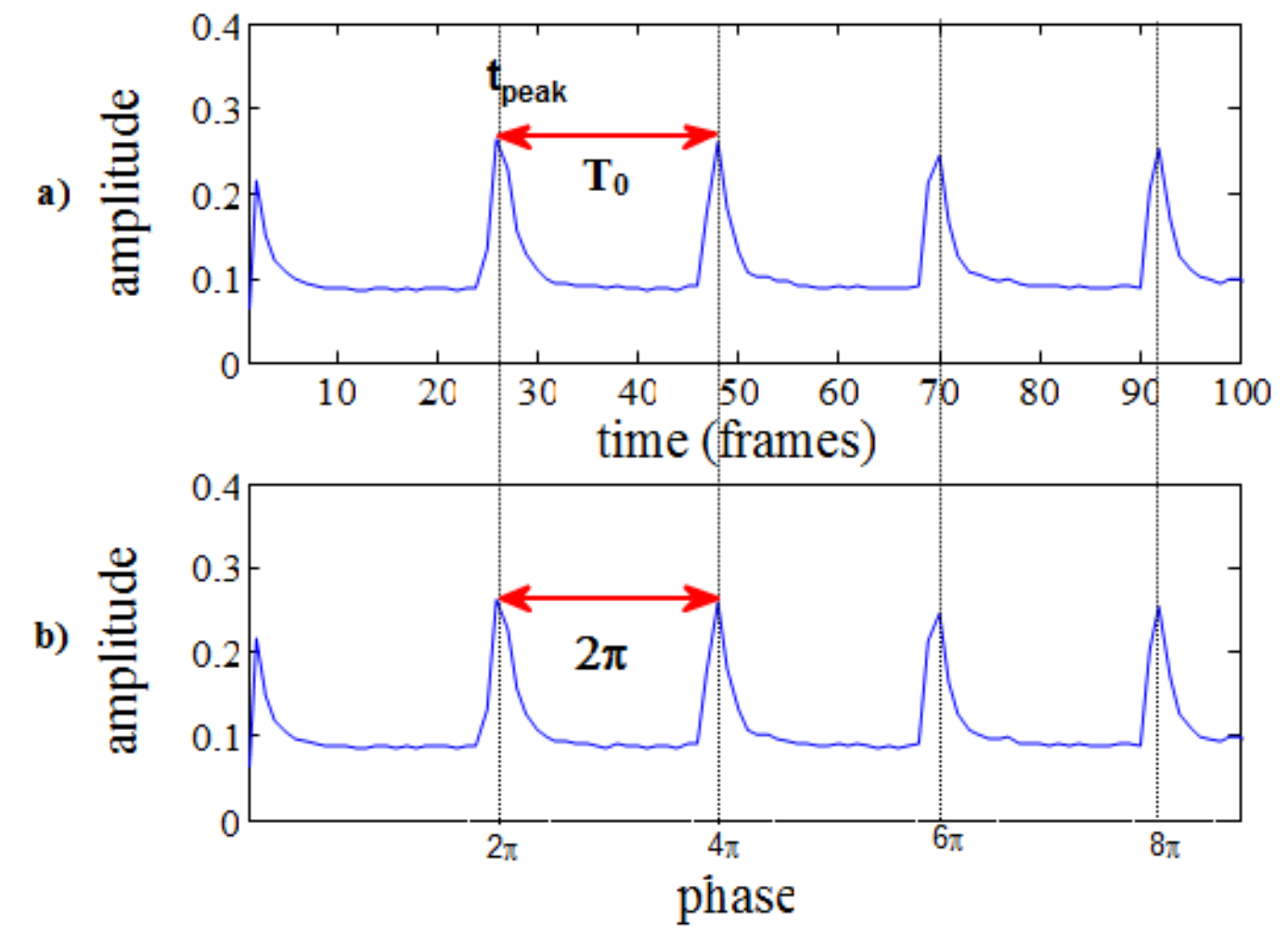

FIG. 2.3: Time-phase conversion. a) A time series showing the successive periods $T_{0}$, each starting at the spiking event $t_{\text {peak }}$. b) The corresponding phase representation of the time series where each period is of size $2 \pi$. 


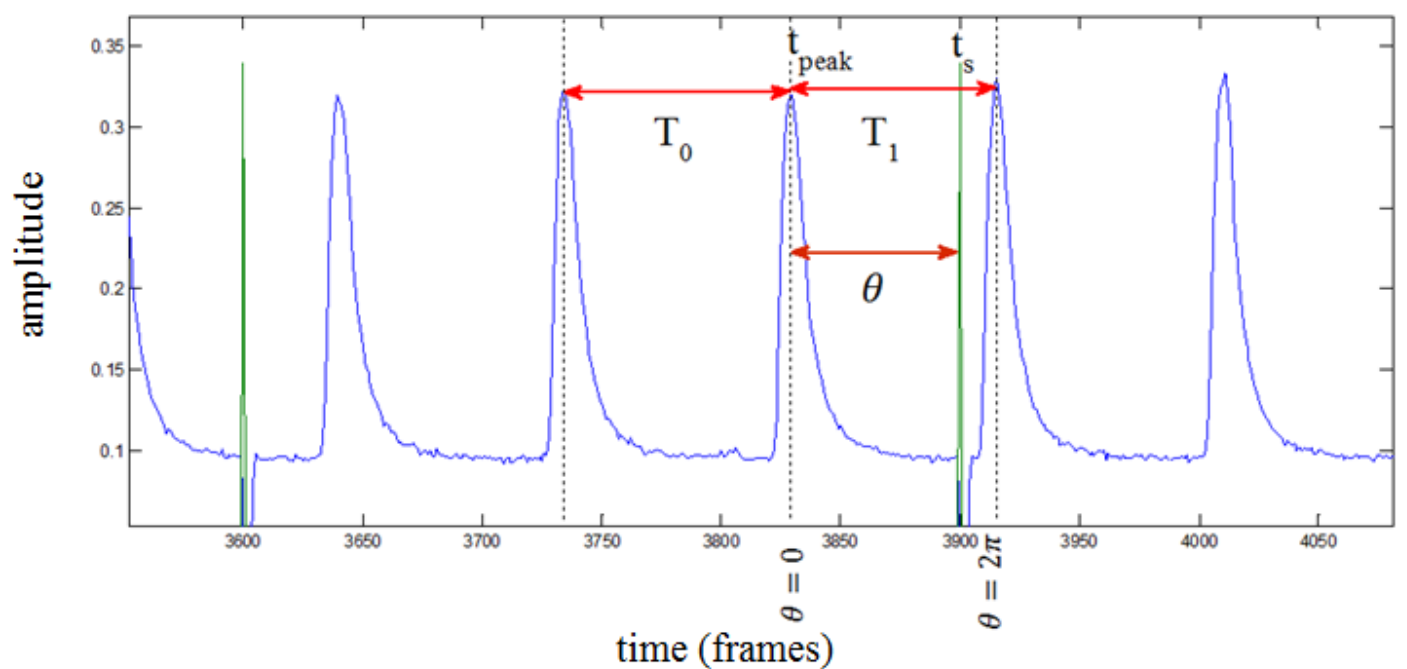

FIG. 2.4: Phase and a phase shift illustration in a phase response curve experiment. Periodic perturbation, $t_{s}$ (green lines), applied at different phase of the cycle $\theta \in[0,2 \pi)$, where $\theta=0$ is set at $t_{\text {peak }}$. The perturbation affects the spiking time, causing phase advancement. This example is from the BelousovZhabotinsky excitatory experiment with a negative perturbation. The natural period was $T_{0}$ and the period shortens to $T_{1}$ after the perturbation.

Pulse perturbations can be described by amplitude and duration variables, which can be adjusted via light control. The amplitude corresponds to a weak perturbation and is confined within a gray scale range $(0-250)$. The duration is a few percent of the autonomous period. Figure 2.5 shows that the interval of successive perturbations includes at least one unperturbed cycle. Differing perturbation intensities and durations cause different magnitudes of the phase shift. The phase shift $\Delta \theta$ is plotted for each perturbed oscillation cycle at a given phase $\theta$ to obtain the PRC. The PRC provides a prediction of an oscillators' phase behavior and its ability to synchronize/desynchronize to external forcing and in feedback-coupled systems [29-33]. 


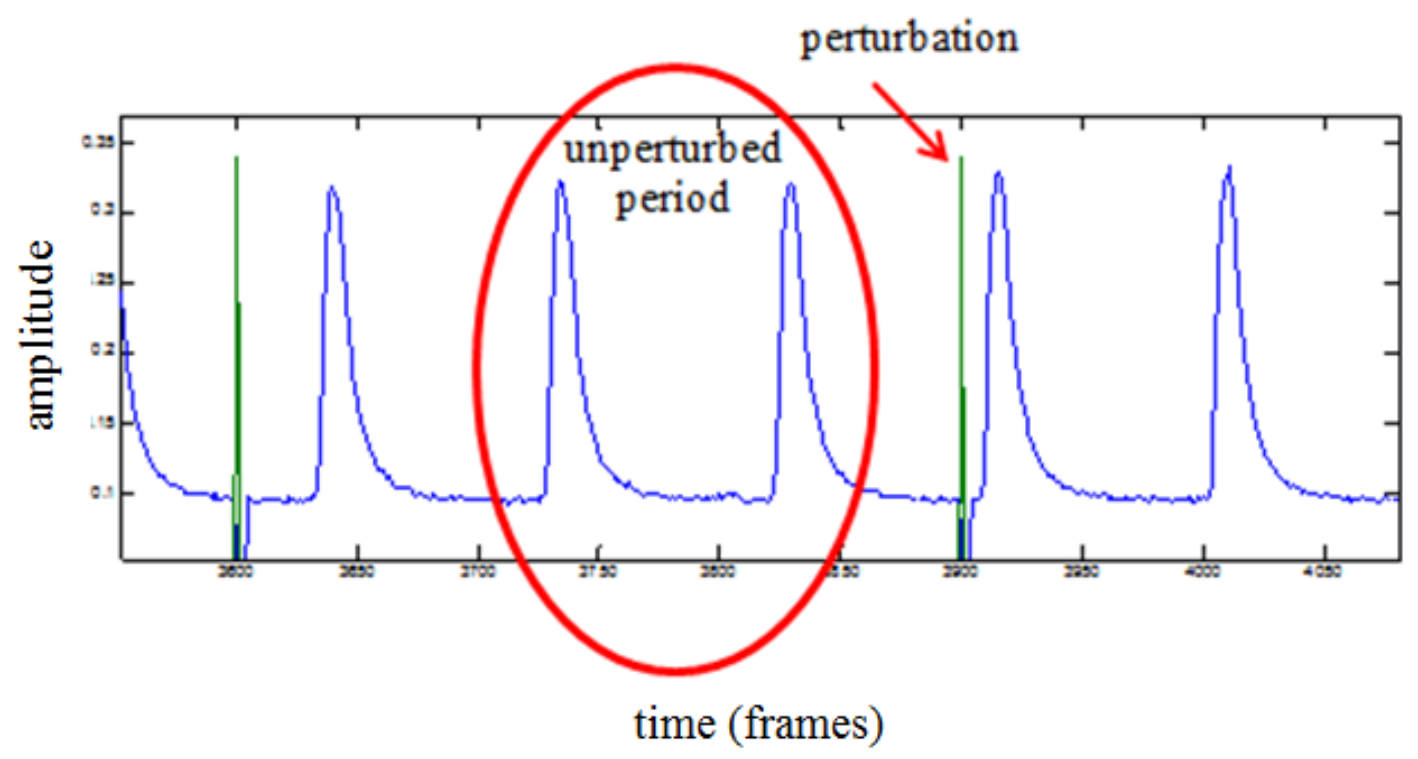

FIG. 2.5: Phase response curve experiment. The interval of successive perturbations (green lines) requires inclusion of at least one unperturbed cycle to define a phase shift according to a prior unperturbed period.

The PRC has been widely used to understand and predict the dynamics in neuronal systems and circadian clocks. An early form of the PRC was reported by Hastings and Sweeney, who studied the light-induced phase shifts in the luminescent organism clock Gonyaulax [34]. The concept of the PRC was further developed by Winfree through his extensive studies of circadian rhythms, which make a complete cycle every 24 hours [35]. Oscillators vary in their response to light perturbations depending on the phase, which can create advancements, delays, or dead zones in the PRC, where no phase shift results. A PRC shape can be classified as type I, monophasic (only advanced phases), as originally described by Hodgkin in 1947 [36], or type II, biphasic (advanced and delayed phase). In simulation of the BZ excitatory model, a positive stimulus results in phase advancing or delaying. However, a negative stimulus only results in phase advancing [21], as shown in Figure 2.6. 


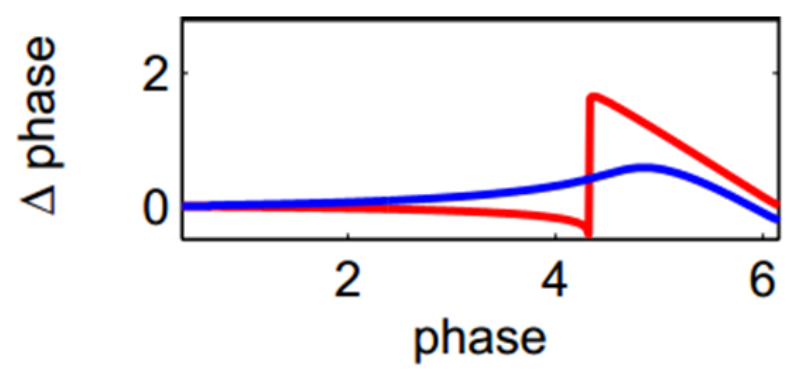

FIG. 2.6: Simulation of dark perturbation (blue plot) and light perturbation (red plot). Plotted data is from ref. [21].

Neuronal models were shown to better synchronize uncoupled oscillators to common inputs when a PRC shape exhibits both phase retardation and advancement compared with only phase advancement. Thus, a type II PRC leads to faster convergence than a type I PRC [37, 38]. In a mathematical representation, convergence and divergence of nearby phases is estimated via the Lyapunov exponent [39]. The absolute value of the Lyapunov exponent corresponds to the rate of convergence or divergence in a synchronization/desynchronization processes. The sign of the Lyapunov exponent, which is represented by the slope of the PRC, determines whether the stimulus will synchronize, desynchronize, or will not affect the oscillations [40]. A negative Lyapunov exponent indicates convergence of nearby trajectories, which leads to synchronization, while a positive Lyapunov exponent indicates divergence of closed trajectories and chaos, which leads to desynchronization. A zero Lyapunov exponent corresponds to no phase behavior difference [39].

\subsubsection{Experimental Setup}

The system used in all experiments in this study involves photosensitive micro-oscillators loaded with the ruthenium BZ catalyst in a catalyst-free BZ solution. The BZ system is 
monitored by a camera imaging system for quantifying the behaviors of interest. The preparations of the loaded particles for the BZ solution and their typical instrumentation are discussed below.

\subsubsection{Preparation of Ruthenium (II) Catalyst Particles}

The particles are made of ion exchange beads (DOWEX 50WX2-100; Sigma-Aldrich, Carlsbad, CA, USA), in which $3.0 \mathrm{~g}$ of the resin was mixed with $1.0 \mathrm{ml}$ of a ruthenium(II) complex $(25.0 \mathrm{mM})$ to obtain the catalyst-loaded particles with a catalyst concentration of $8.33 \times 10^{-6} \mathrm{~mol} / \mathrm{g}$ resin. The mixture was stirred for 24 hours, and the particles were then washed with distilled water and filtered before use. The ruthenium (II) complex solution was previously prepared by dissolving $0.468 \mathrm{~g}$ of tris(2,2-bipyridine) ruthenium(II) chloride hexahydrate, $\left[\mathrm{Ru}(\mathrm{bpy})_{3}\right] \mathrm{Cl}_{2} \cdot 6 \mathrm{H}_{2} \mathrm{O}$, in $25.0 \mathrm{ml}$ of distilled water, which was kept in a refrigerator prior to use.

\subsubsection{Preparation of Catalyst-Free BZ Solution}

The BZ solution was obtained by preparing a mixture of reactants to ensure an excitatory oscillatory medium for the individual particles. The catalyst-free solution consisted of $0.08 \mathrm{M}$ malonic acid $(\mathrm{MA}), 0.02 \mathrm{M}$ sodium bromide $(\mathrm{NaBr}), 0.78 \mathrm{M}$ sulfuric acid $\left(\mathrm{H}_{2} \mathrm{SO}_{4}\right)$, and $0.48 \mathrm{M}$ sodium bromate $\left(\mathrm{NaBrO}_{3}\right)$. The solution was prepared in a $25.0 \mathrm{ml}$ flask for each experiment from fresh malonic acid and stock prepared solutions, and was used after ten minutes. The stock solutions were $1.0 \mathrm{M} \mathrm{NaBr}, 3.0 \mathrm{M} \mathrm{H}_{2} \mathrm{SO}_{4}$, and 2.0 $\mathrm{M} \mathrm{NaBrO}_{3}$. All chemicals were purchased from Fisher (Fair Lawn, NJ, USA). 


\subsubsection{Instrumental Design}

Catalyst loaded particles were set in a Plexiglas vessel of $35.0 \mathrm{~mm}$ diameter and $12.0 \mathrm{~mm}$ height, and then immersed in $3.0 \mathrm{~mm}$ of catalyst-free BZ solution. The light was projected into the reaction vessel by a spatial light modulator (SLM), consisting of a projector, band pass filter (440 - $460 \mathrm{~nm})$, and beam splitter. Real-time imaging using a CCD camera with a PC computer was then used to record background and bead illumination intensities resulting from a periodically changing catalyst redox state. The experimental setup is illustrated in Figure 2.7.

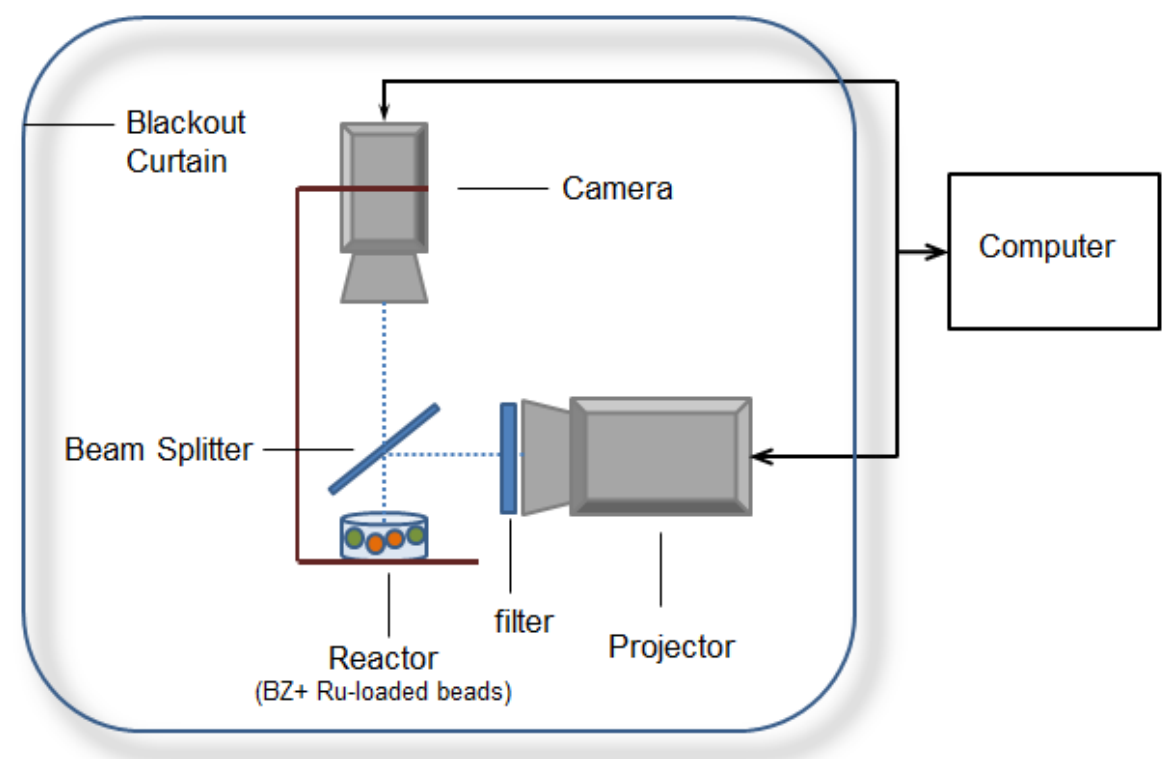

FIG. 2.7: Experimental setup. The light is supplied from the projector only, and is filtered and reflected by a beam splitter onto the ruthenium-loaded particles. Images are recorded in real time by the camera and processed via a computer.

\subsubsection{PRC Experimental Technique and Procedures}

Two experiments were performed using positive or negative perturbations to obtain the PRC of the individual BZ oscillators. Positive perturbations involved applying a periodic light 
intensity that was above the baseline light intensity for natural oscillations, whereas for negative perturbations, a periodic light intensity below the baseline light intensity of natural oscillations was applied. The reference baseline intensity was chosen as $I_{0}=1.67 \mathrm{~mW} \mathrm{~cm}^{-2}$, which corresponds to a gray scale of 180 [41]. In the reactor vessel, the particles were kept at least three diameters apart to prevent diffusive coupling, as shown in Figure 2.8. The positive/negative perturbations relative to the background intensity were introduced by setting the light intensity to its highest/lowest value on the gray scale (250 and 0, respectively). Each perturbation of determined amplitude lasted for $2.0 \mathrm{~s}$, after which the background intensity of $I_{0}$ was restored. The light intensity of gray scale 250 corresponded to $I_{0}=2.31 \mathrm{~mW} \mathrm{~cm}^{-2}$. The perturbation interval was chosen to include more than twice the averaged period of the oscillators for each experiment. The interval in the positive-perturbation experiment was chosen as $300.0 \mathrm{~s}$, with an averaged period of the oscillators of $107.5 \mathrm{~s}$. The interval in the negative-perturbation experiment was $150.0 \mathrm{~s}$, with an averaged period of the oscillators of $65.0 \mathrm{~s}$. The phase change was calculated after each perturbation to plot a phase change $v s$. phase cycle $(0-2 \pi)$ graph. The total data set is shown in Figure 2.9a. The data points of phases were averaged, and a mean phase change was obtained to represent the smoothed PRC for the oscillators, as shown in Figure 2.9b.

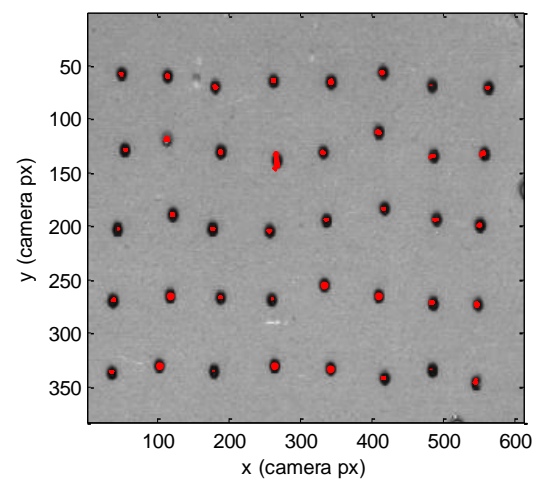

FIG. 2.8: Arrangement of catalyst particles in a catalyst-free Belousov-Zhabotinsky solution to maintain a discrete uncoupled system. 
a)
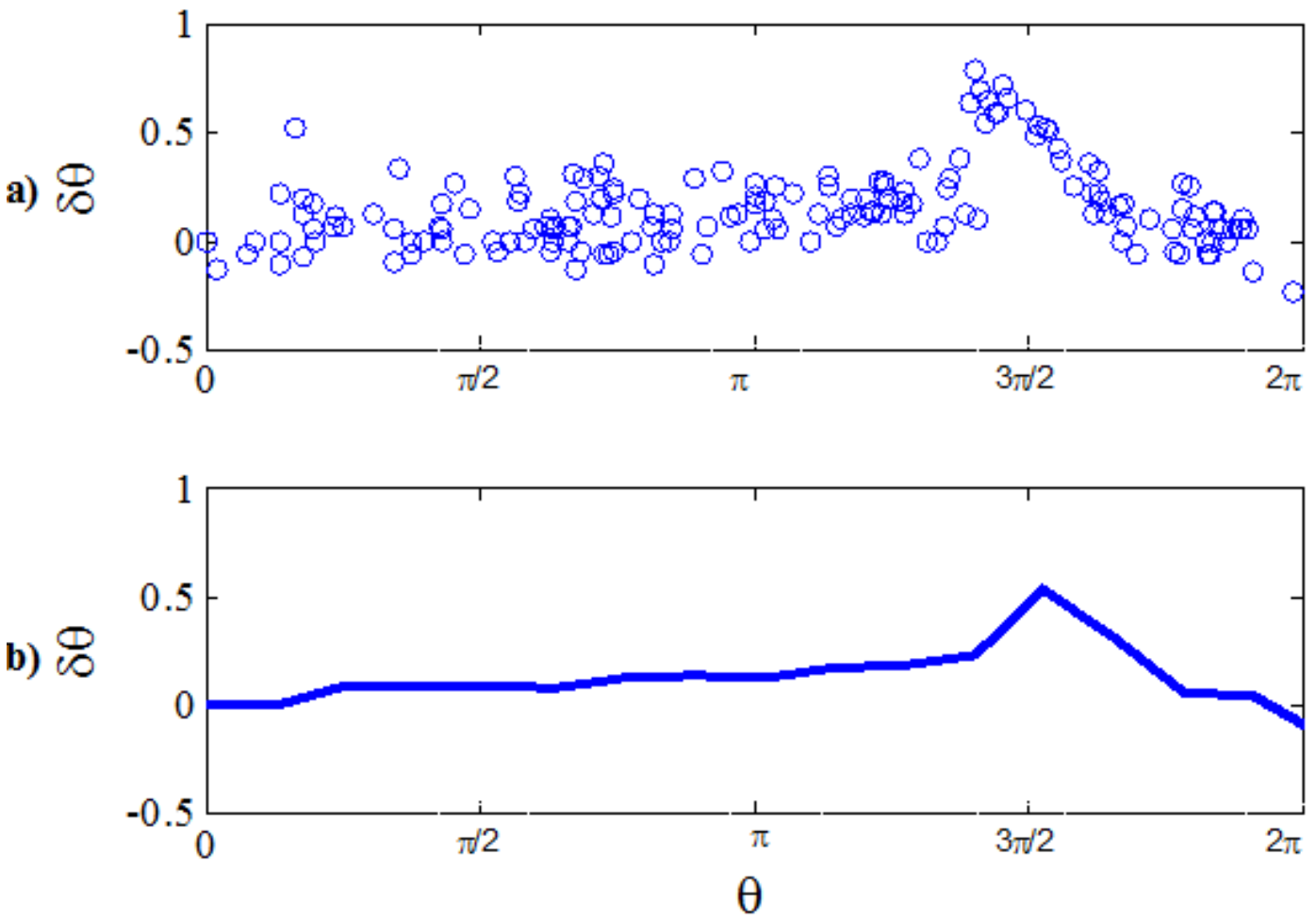

FIG. 2.9: An averaged and smoothed PRC of several oscillators. a) A phase response curve (PRC) of 19 individual oscillators with negative perturbations. b) The smoothed PRC from 19 beads in the PRC experiment.

\subsubsection{Experimental Results}

The PRC experiments showed that both positive and negative perturbations lead to phase advancement of the spiking-time, $\delta \theta>0$. For negative perturbations, Figure 2.10a shows that only a positive region was observed because of phase advancement, as previously reported in a simulation [21]. This one phasic PRC is classified as type (I), which was found experimentally by Gutkin et al. and in a model of cortical neurons [42]. Positive perturbations, however, resulted in a discontinuity that separates the positive region from a dead zone where no phase changes are observed, $\delta \theta=0$. Unlike simulation of BZ excitatory system with positive perturbations [21], which produced a small region of retardation, $\delta \theta<0$, Figure $2.10 \mathrm{~b}$ shows that no negative 
region was detectable in the experiment. This simulated PRC is identified as type (II), which was resolved in the experimental results of positive perturbations to non-cortical neurons [30], as shown in Figure 2.10c.

a)

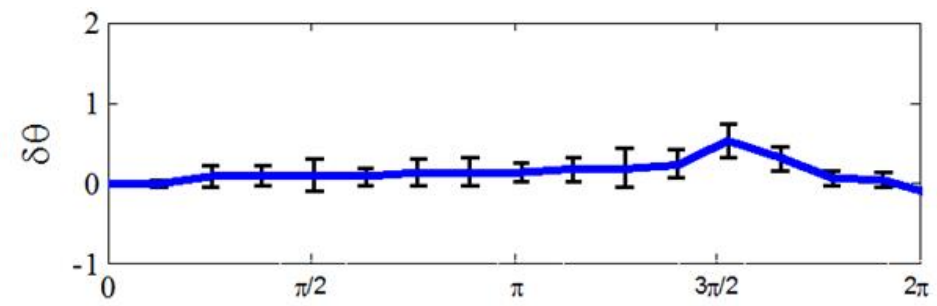

b)

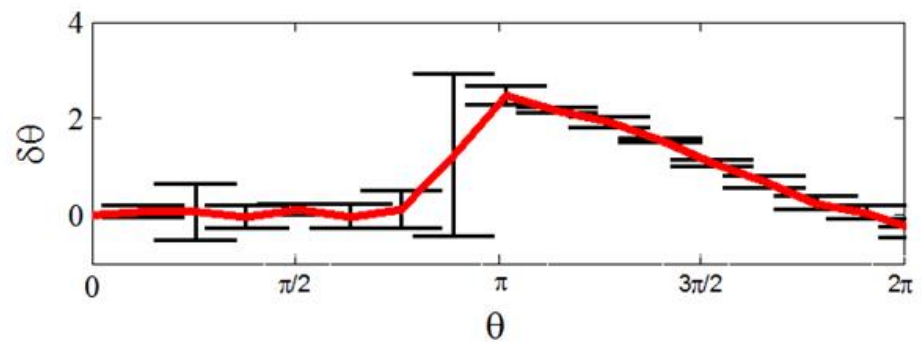

c)

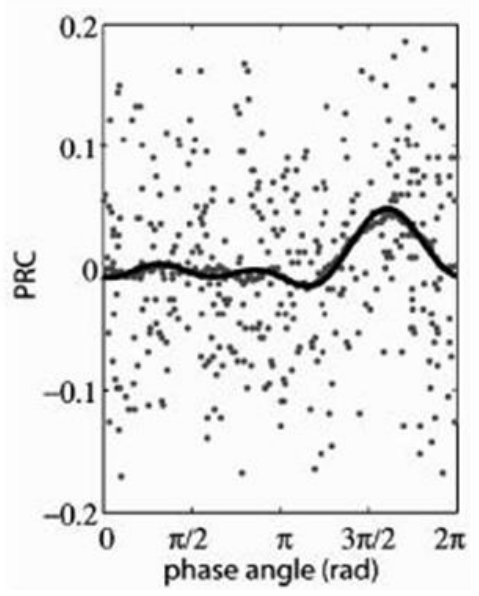

FIG. 2.10: PRC from negative and positive perturbations. a) Experimental data from negative perturbation (blue plot), $I=-1.67 \mathrm{~mW} \mathrm{~cm}^{-2}$ below the background intensity. The standard errors are indicated by black bars. b) Experimental data from positive perturbation (red plot), $I=0.65 \mathrm{~mW} \mathrm{~cm}^{-2}$ above the background intensity. The standard errors are indicated by black bars. c) Simulation of dark perturbation (black plot) of neurons fitted to data points and averaged (gray plot) from the experiment. The plotted data set (c) is from ref. [42]. 
All previous observations were obtained from the excitatory photosensitive system, where increasing light intensity enhances the autocatalytic production of activator species, $\mathrm{HBrO}_{2}$, as seen in by Eq. 2.15 and Eq. 2.34 in Section 2.1.1 and 2.1.3, respectively. The strength of the perturbation is chosen to be the largest possible magnitude in the gray scale in order to match with target experiments in Chapter 3. The negative and positive values of the stimulus relative to the chosen background lead to the different classes of PRC. The detailed observations based on the BZ mechanism are as follows:

In the negative perturbation experiments, only phase advancement was found experimentally. Phase advancement represents shortening of the period at which the perturbation is applied in reference to the preceding period. This happens when process B is turned on as the activator/inhibitor reaches the critical threshold, as previously reported in Section 2.1.1. In a photosensitive excitatory system, the light intensity primarily affects $\mathrm{HBrO}_{2}$ production. Decreasing the light intensity will produce less $\mathrm{HBrO}_{2}$ via alterations of the process of catalyst oxidation and bromate reduction, as shown in Eq. 2.15. The inhibitor species is merely generated via process $\mathrm{C}$ and removed via process $\mathrm{A}$. Thus, the observation of only a positive region is an indication of the release of $\mathrm{HBrO}_{2}$ and the effective removal of $\mathrm{Br}^{-}$as governed by Eq. 2.2 in process A. The positive region is small and shifted to the end of the cycle, indicating that activator production is small and is taking a longer time to reach the critical threshold, which delays the firing event via process $B$. The maximum advancement of a phase occurred near $3 \pi / 2$. Therefore, the PRC results produced by negative perturbations can be attributed to maintaining the cycle for a longer time in process A.

In the positive perturbation experiment, $I=0.65 \mathrm{~mW} \mathrm{~cm}^{-2}$ is chosen to be a perturbation greater than the background intensity of $I_{0}$. The region of discontinuity is the region between $\frac{3 \pi}{4}$ 
and $\pi$. Phase advancement occurred at perturbations between $\pi$ and $2 \pi$ of the oscillation cycle. The maximum advancement of a phase occurred near $\pi$. The dead zone is represented by a zero line region in the PRC. In the dead zone, perturbations occurred in the refractory phase of oscillation. Such perturbations had no effects on the period early in the phase cycle. In addition, with high light intensity, the photochemical production of $\mathrm{HBrO}_{2}$ from bromate via Eq. 2.34 can be offset by the competitive reaction of photochemical production of $\mathrm{Br}^{-}$from bromomalonic acid via Eq. 2.35, which prevents activator accumulation from the beginning of the cycle. Phase retardation represents the lengthening of the stimulated period in reference to the preceding nonstimulated period. Phase retardation was not observed in the experiment, while the simulation indicated a small region of delayed phases before the discontinuity. The appearance of a negative region in the PRC indicates the presence of $\mathrm{Br}^{-}$above its critical threshold in this region before the discontinuity. $\mathrm{Br}^{-}$is removed rapidly when the autocatalysis is switched on. Phase advancement results near the last half of the phase cycle as the autocatalytic process produces more activator species. The discontinuity appears with the instantaneous buildup of the activator, the fast variable. This increase in activator species is promoted by positive perturbations in a photosensitive system via Eq. 2.15. The size and position of the discontinuity on the phase cycle depends on the size of perturbations. Larger perturbations lead to a larger positive region, which is in agreement with models of biological oscillators [43] and BZ modeling [21]. The position of the positive region shifts to earlier regions with a higher perturbation size. Figure 2.11 shows that the maximum advancement of a phase by larger $\left(I=0.65 \mathrm{~mW} \mathrm{~cm}^{-2}\right)$ and smaller $(I=$ $0.19 \mathrm{~mW} \mathrm{~cm}^{-2}$ ) positive perturbations occurred near $\pi$ and $\frac{3 \pi}{2}$, respectively. 

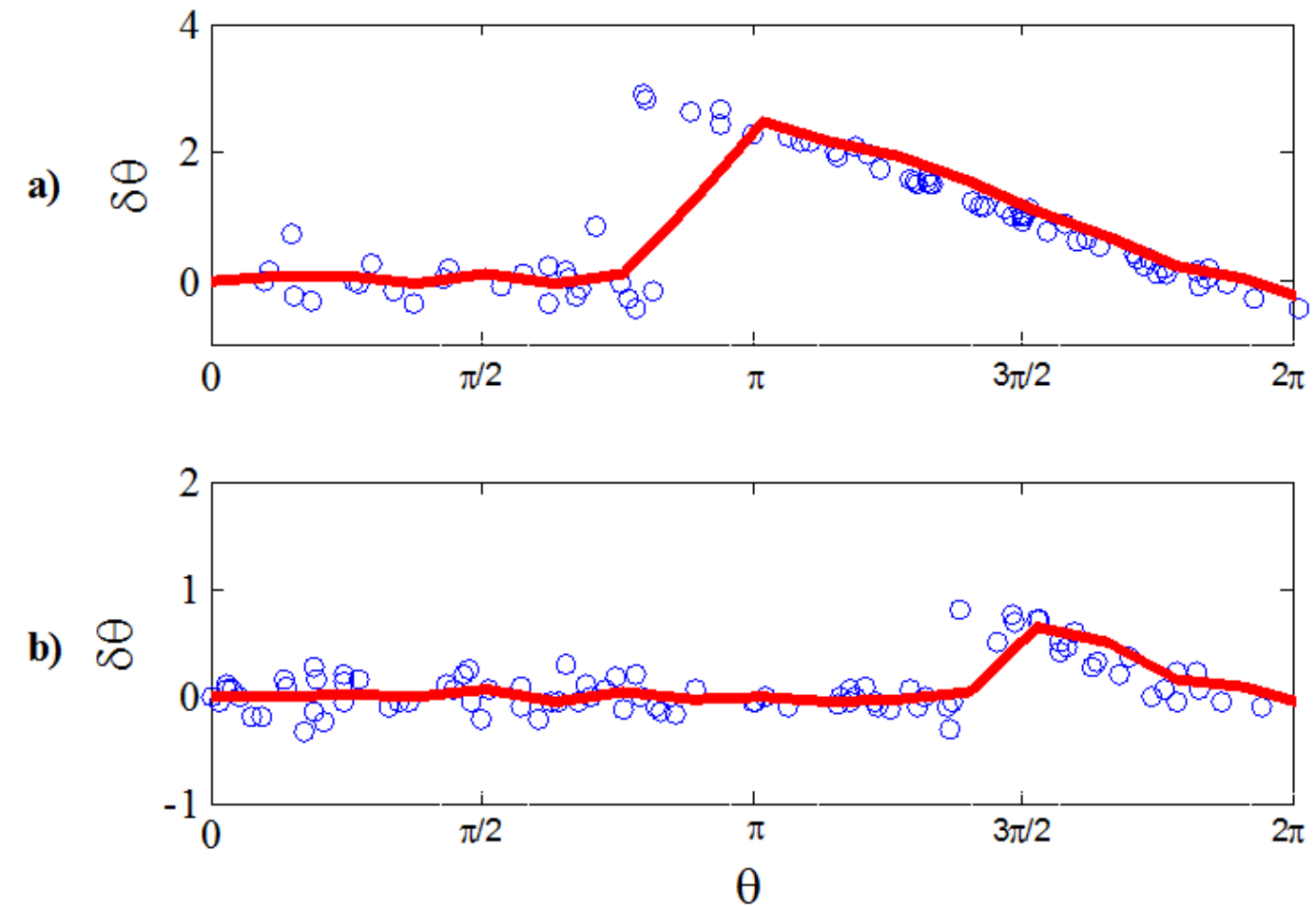

FIG. 2.11: PRC with different positive perturbation sizes. a) Data points (blue circles) and averaged points (red plot) from experiment with positive perturbation, $I=0.65 \mathrm{~mW} \mathrm{~cm}^{-2}$. b) Data points (blue circles) and averaged points (red plot) from experiment with positive perturbation $I=0.19 \mathrm{~mW}^{-2}$.

By comparison to the simulation [21], increasing the size of perturbations shifts the advanced region to occur earlier in the cycle than that in the simulation, which may overcome any delayed region that can be observed experimentally. The excess of the activator effectively removed the inhibitor in Eq. 2.3, where the photosensitive system with positive perturbations enhances the autocatalytic process at the expense of inhibitor production. In addition, the small region delayed by smaller positive perturbations is difficult to resolve because of the interference of noise in the experiment. However, the discontinuity is an indication of the presence of this unresolved feature in the PRC, in comparison with the monophasic PRC by negative perturbations, as shown in Figure 2.9a. 
The main objective of these experiments is to determine the type of perturbation suitable for synchronization or desynchronization based on the shape of the PRCs. In addition, PRCs provide information on the time of perturbation during the phase cycle, as discussed in Chapter 3. 


\section{References}

[1] J. N. Demas and D. Diemente, "An oscillating chemical reaction with a luminescent indicator.” J. Chem. Educ. 50, 357 (1973).

Online Version

[2] V. Gáspár, G. Bazsa, and M. Beck, "The influence of visible-light on the BelousovZhabotinskii oscillating reactions applying different catalysts." Z. Phys. ChemieLeipzig 264, 43-48 (1983).

Online Version

[3] J. M. Bodet, J. Ross, and C. Vidal, "Experiments on phase diffusion waves." J. Chem. Phys. 86, 4418-4424 (1987).

Online Version

[4] M. K. R. Reddy, Z. Nagy-Ungvarai, and S. C. Müller, "Effect of visible light on wave propagation in the ruthenium-catalyzed Belousov-Zhabotinsky reaction.” J. Phys. Chem. 98, 12255-12259 (1994).

Online Version

[5] H.-J. Krug, H. Brandtstaedter, and L. Pohlmann, "Nucleation and wave propagation in the modified oregonator and comparison with experiments in a photosensitive Belousov-Zhabotinsky gel reactor.” J. Phys. Chem. 99, 10237-10245 (1995).

Online Version

[6] T. Amemiya, S. Kádár, P. Kettunen, and K. Showalter, "Spiral wave formation in threedimensional excitable media." Phys. Rev. Lett. 77, 3244-3247 (1996).

Online Version 
[7] M. Markus, G. Kloss, and I. Kusch, "Disordered waves in a homogeneous, motionless excitable medium." Nature 371, 402-404 (1994).

Online Version

[8] K. I. Agladze, V. A. Davydov, and A. S. Mikhaǐlov, "Observation of a helical-wave resonance in an excitable distributed medium.” JETPL 45, 767 (1987).

Online Version

[9] K. Agladze, Á. Tóth, T. Ichino, and K. Yoshikawa, "Propagation of chemical waves at the boundary of excitable and inhibitory fields." J. Phys. Chem. A 104, 6677-6680 (2000).

Online Version

[10] O. Steinbock, V. Zykov, and S. C. Müller, "Control of spiral-wave dynamics in active media by periodic modulation of excitability." Nature 366, 322-324 (1993).

Online Version

[11] T. Amemiya, P. Kettunen, S. Kadar, T. Yamaguchi, and K. Showalter, "Formation and evolution of scroll waves in photosensitive excitable media.” Chaos 8, 872-878 (1998). Online Version

[12] R. Toth and A. F. Taylor, “"'The tris(2,2'-bipyridyl)ruthenium-catalysed BelousovZhabotinsky reaction. Prog .React. Kinet. Mech. 31, 59-115 (2006).

Online Version

[13] R. M. Noyes, R. Field, and E. Körös, “Oscillations in chemical systems. I. Detailed mechanism in a system showing temporal oscillations.” J. Am. Chem. Soc. 94, 13941395 (1972).

Online Version 
[14] R. J. Field and R. M. Noyes, "Oscillations in chemical systems. IV. Limit cycle behavior in a model of a real chemical reaction.” J. Chem. Phys. 60, 1877-1884 (1974). Online Version

[15] A. M. Zhabotinsky, F. Buchholtz, A. B. Kiyatkin, and I. R. Epstein, "Oscillations and waves in metal-ion-catalyzed bromate oscillating reactions in highly oxidized states." J. Phys. Chem. 97, 7578-7584 (1993).

Online Version

[16] H. Ševčíková, I. Schreiber, and M. Marek, "Dynamics of oxidation Belousov-Zhabotinsky waves in an electric field.” J. Phys. Chem. 100, 19153-19164 (1996).

Online Version

[17] H. J. Krug, L. Pohlmann, and L. Kuhnert, "Analysis of the modified complete Oregonator accounting for oxygen sensitivity and photosensitivity of BelousovZhabotinsky systems.” J. Phys. Chem. 94, 4862-4866 (1990).

Online Version

[18] R. J. Field and R. M. Noyes, "Oscillations in chemical systems. V. Quantitative explanation of band migration in the Belousov-Zhabotinskii reaction.” J. Am. Chem. Soc. 96, 2001-2006 (1974).

Online Version

[19] A. L. Belmonte, Q. Ouyang, and J.-M. Flesselles, "Experimental survey of spiral dynamics in the Belousov-Zhabotinsky reaction.” J. Phys. II France 7, 1425-1468 (1997).

Online Version 
[20] I. Hanazaki, Y. Mori, T. Sekiguchi, and G. Rábai, "Photo-response of chemical oscillators.” Phys. D 84, 228-237 (1995).

Online Version

[21] M. R. Tinsley, S. Nkomo, and K. Showalter, "Chimera and phase-cluster states in populations of coupled chemical oscillators.” Nat. Phys. 8, 662-665 (2012).

Online Version

[22] S. Nkomo, M. R. Tinsley, and K. Showalter, "Chimera states in populations of nonlocally coupled chemical oscillators.” Phys. Rev. Lett. 110, 244102 (2013).

Online Version

[23] E. B. Robertson and H. B. Dunford, "The state of the proton in aqueous sulfuric acid." J. Am. Chem. Soc. 86, 5080-5089 (1964).

Online Version

[24] R. Toth, A. F. Taylor, and M. R. Tinsley, "Collective behavior of a population of chemically coupled oscillators.” J. Phys. Chem. B 110, 10170-10176 (2006).

Online Version

[25] S. Kádár, T. Amemiya, and K. Showalter, "Reaction mechanism for light sensitivity of the Ru(bpy) ${ }_{3}{ }^{2+}$-catalyzed Belousov-Zhabotinsky reaction.” J. Phys. Chem. A 101, 8200-8206 (1997).

Online Version

[26] A. F. Taylor, P. Kapetanopoulos, B. J. Whitaker, R. Toth, L. Bull, and M. R. Tinsley, "Phase clustering in globally coupled photochemical oscillators." Eur. Phys. J. Spec. Top. 165, 137-149 (2008).

Online Version 
[27] V. K. Vanag, L. Yang, M. Dolnik, A. Zhabotinsky, and I. R. Epstein, "Oscillatory cluster patterns in a homogeneous chemical system with global feedback." Nature 406, 389-391 (2000).

Online Version

[28] S. A. Oprisan, "A Geometric approach to phase resetting estimation based on mapping temporal to geometric phase." In Phase Response Curves in Neuroscience, edited by N. W. Schultheiss, A. A. Prinz, and R. J. Butera (Springer, New York, 2012), pp. 131162.

Online Version

[29] T. I. Netoff, M. I. Banks, A. D. Dorval, C. D. Acker, J. S. Haas, N. Kopell, and J. A. White, "Synchronization in hybrid neuronal networks of the hippocampal formation." J. Neurophysiol. 93, 1197-1208 (2005).

Online Version

[30] R. F. Galán, G. B. Ermentrout, and N. N. Urban, "Efficient estimation of phaseresetting curves in real neurons and its significance for neural-network modeling." Phys. Rev. Lett. 94, 158101 (2005).

Online Version

[31] T. I. Netoff, C. D. Acker, J. C. Bettencourt, and J. A. White, "Beyond two-cell networks: Experimental measurement of neuronal responses to multiple synaptic inputs.” J. Comput. Neurosci. 18, 287-295 (2005).

Online Version

[32] I. Z. Kiss, Y. Zhai, and J. L. Hudson, "Predicting mutual entrainment of oscillators with experiment-based phase models.” Phys. Rev. Lett. 94, 248301 (2005). 
Online Version

[33] I. Z. Kiss, C. G. Rusin, H. Kori, and J. L. Hudson, "Engineering complex dynamical structures: Sequential patterns and desynchronization.” Science 316, 1886-1889 (2007).

Online Version

[34] J. W. Hastings and B. M. Sweeney, "A persistent diurnal rhythm of luminescence in Gonyaulax Polyedra.” Biol. Bull. 115, 440-458 (1958).

Online Version

[35] J. J. Tyson and L. Glass, “Arthur T. Winfree (1942-2002).” J. Theor. Biol. 230, 433439 (2004).

Online Version

[36] A. L. Hodgkin, "The local electric changes associated with repetitive action in a nonmedullated axon.” J. Physiol. 107, 165-181 (1948).

Online Version

[37] A. Abouzeid and B. Ermentrout, "Type-II phase resetting curve is optimal for stochastic synchrony.” Phys. Rev. E 80, 011911 (2009).

Online Version

[38] S. Marella and G. B. Ermentrout, "Class-II neurons display a higher degree of stochastic synchronization than class-I neurons.” Phys. Rev. E 77, 041918 (2008) Online Version

[39] A. Pikovsky, M. Rosenblum, and J. Kurths, Synchronization: A universal concept in nonlinear sciences (Cambridge University Press, Cambridge, 2003).

Online Version 
[40] C. J. Wilson, B. Beverlin, and T. Netoff, "Chaotic desynchronization as the therapeutic mechanism of deep brain stimulation.” Front. Syst. Neurosci. 5. 00050 (2011). Online Version

[41] C. Xia and N. Fang, "Fully three-dimensional microfabrication with a grayscale polymeric self-sacrificial structure.” J. Micromech. Microeng. 19, 115029 (2009). Online Version

[42] B. S. Gutkin, G. B. Ermentrout, and A. D. Reyes. "Phase-response curves give the responses of neurons to transient inputs." J. Neurophysiol. 94, 1623-1635 (2005).

Online Version

[43] L. Glass, and A. T. Winfree. "Discontinuities in phase-resetting experiments." Am. J. Physiol. 246, R251-R258 (1984).

Online Version 


\section{Chapter 3}

\section{Synchronization and Desynchronization in Populations of Uncoupled Chemical Oscillators with Periodic Forcing}

\subsection{Introduction}

Desynchronization is a method for reestablishing normal neuronal function following pathological synchrony. According to the gate theory [1], controlling the flow of signals to the brain via a neurological gate depends on the activation medium of spinal cord nerves and other neural circuits in the brain [2]. Signals conveyed by a higher activation medium can be blocked, whereas signals conveyed by a smaller activated medium pass through the gate [1]. In this sense, neuronal signaling that is related to mental, emotional, or physical activities becomes distorted because of increased neuronal activities and synchronization, which further leads to blocked signals. Indeed, rhythmic output by the basal ganglia correlates with abnormal activities [3-5], such as tremors in Parkinson's disease (PD) [6-9]. Some studies have therefore focused on synchrony regulation to treat neural diseases. Deep brain stimulation (DBS), a neurosurgical therapy, has been suggested to relieve symptoms of PD through a desynchronization mechanism [10-12] rather than an entrainment mechanism [13-15]. Desynchronization has been theoretically verified via a mechanism that induces unlearned synaptic interactions among neural networks $[16,17]$. DBS has been broadly used as a high-frequency, pulsatile signal and as an open-loop controller. Low-energy control methods have been validated numerically to model desynchronization of a population of neurons [18-20]. These methods consume less energy and may cause less tissue damage than the currently available DBS methods. 
Desynchronization of coupled oscillators can be controlled via a feedback approach or by regulating the timing of external forcing. Effective desynchronization of the bursting neuron model was theoretically demonstrated via time-delayed feedback [21]. Desynchronization and cluster formation of coupled and synchronized electrochemical oscillators has been experimentally examined by applying time-delayed feedback [22]. For an external forcing approach, the perturbation of heart tissue by an electrical pulse can lead to rhythm desynchronization if the pulse is delivered at certain phases after the refractory phase [23]. The combination of feedback control with external forcing methods has been examined, and computational studies show that perturbations of double pulses after synchronization lead to desynchronized oscillators, even in the presence of noise [11]. A model of a synchronized neuronal population was theoretically examined and showed desynchronization through an intermediate cluster state [24]. The model was modified to use the properties of adaptive neuronal firing via spike timing-dependent plasticity [25]. In this context, pathological connections speed up abnormal neuronal synchrony (kindling), while controlled desynchronization methods induce anti-kindling. Desynchronization involves splitting the synchronized population into clusters by lowering the rate of coincident firing. This model has been supported by inducing desynchronization in epileptic rats [16]. Optimal desynchronization with a single input has been numerically examined, and this method leads to exponential desynchronization of coupled oscillators [19].

Our method uses an adaptive stimulus with periodic single pulses applied to a population of uncoupled BZ oscillators. The perturbation's frequency is within the time scale of an oscillator's frequency. Perturbations are applied in a controlled manner by choosing the appropriate timing during the phase cycle, in contrast to the common open-loop application of 
DBS. This chapter discusses experimental and simulation studies of desynchronization of synchronized oscillators. The experiments are based on information from previous PRC experiments, which were discussed in the previous chapter.

\subsection{Synchronization and Desynchronization Measurements}

External forcing experiments involve constructing synchronized signals and then forcing them to desynchronize. The degree of synchronization can be determined by a combination of approaches. One approach involves appropriate external forcing based on measurements from PRC experiments and qualitative consideration of the Lyapunov exponent. Then, the degree of phase coherence is measured by qualitatively evaluating the mean signal of the time series and quantitatively measuring the order parameter, $r$. This approach is discussed below.

\subsubsection{Lyapunov Exponent and the Phase Response Curve}

The prediction of synchronization or desynchronization by external perturbations can be made by analyzing the shape of the PRC. The PRC in Figure 3.1a shows that a positive perturbation has only a negative slope (excluding regions of discontinuity). Negative perturbations, however, have both positive and negative slopes (blue plot), as shown in Figure 3.1b. 

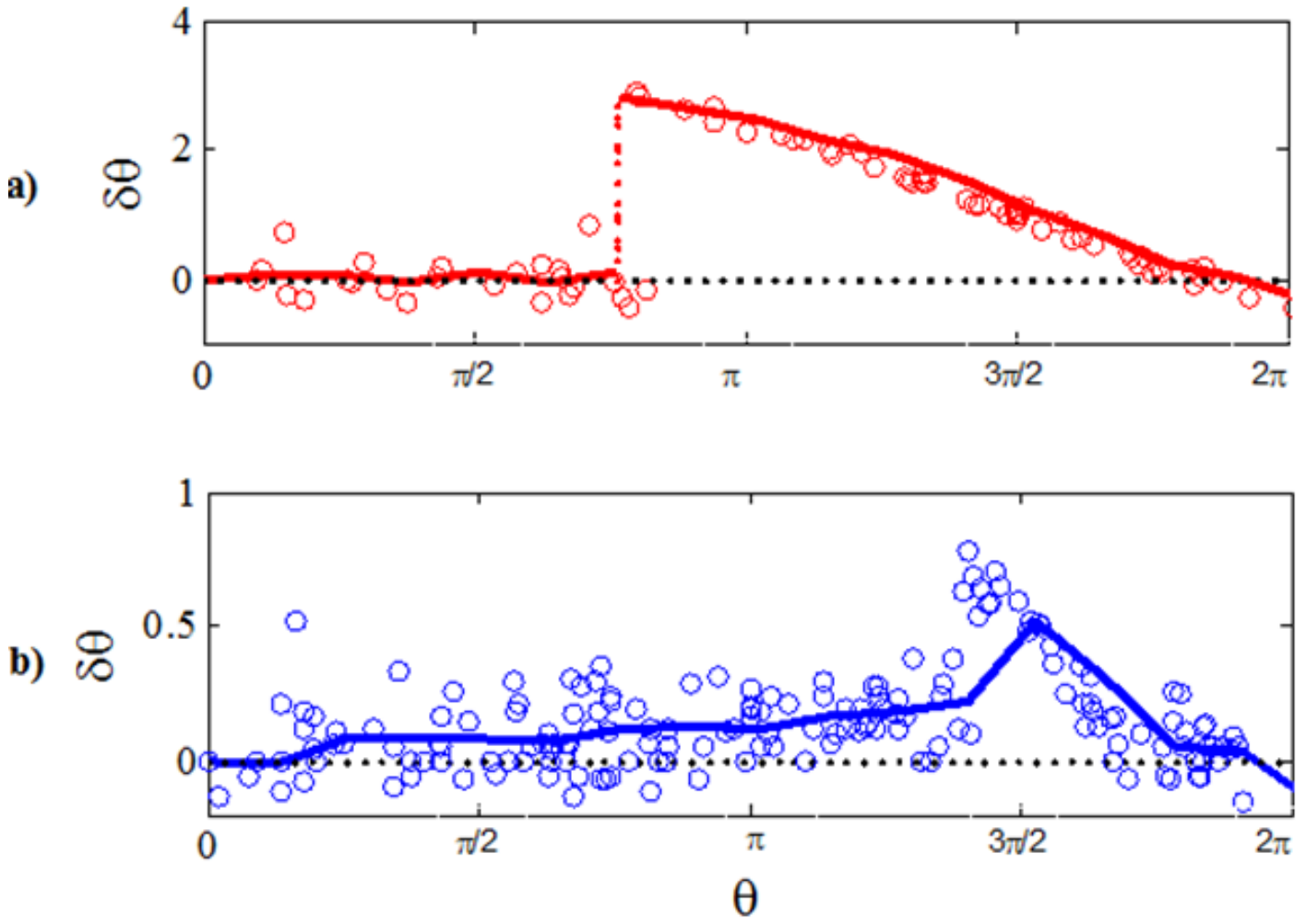

FIG. 3.1: Phase response curves. a) Positive perturbations result in a PRC with only a negative slope, which can be seen over the last half of the cycle. b) Negative perturbations result in a PRC with positive and negative slopes, where the first half of the cycle exhibits a small positive slope.

The optimal stimulus to entrain uncoupled oscillators was mathematically developed by Wilson and Moehlis [26] in 2014 to model the circadian activity of Drosophila by referring to the value of the Lyapunov exponent [27]. If the Lyapunov exponent is negative, each nearby oscillator will converge at an exponential rate, leading to synchronization with the applied stimulus. However, if the Lyapunov exponent is positive, oscillators with nearby phases will exponentially diverge and lead to desynchronization with the applied stimulus [28]. The phase rate change resulting from a perturbation of an oscillator of phase $\theta$ with a natural frequency $\omega$ was defined by Brown et al. [29] as follows:

$$
\dot{\theta}=\omega+Z(\theta) I(t)
$$


where the oscillator's phase response curve is defined as $Z(\theta)$ by a stimulus $I(t)$. The Lyapunov exponent is related to the slope of the PRC. The expression for the finite time Lyapunov exponent, $\Lambda$, over a period $T$ for an oscillator undergoing negative perturbations, $I_{n e g}$, with the phase response curve $Z_{\text {neg }}(\theta)$, is given by the following [19]:

$$
\Lambda=\frac{1}{T} \int_{0}^{T}\left(Z^{\prime}{ }_{n e g}(\theta) I_{n e g}(t)\right) d t
$$

where $^{\prime}=d / d \theta$ is the slope of the PRC with negative perturbations. If the slope is positive, $\left(Z^{\prime}{ }_{n e g}(\theta) I_{n e g}(t)\right)>0$, then the finite time Lyapunov exponent is positive, indicating desynchronization. The phase separation of two oscillators, $\Phi$, in terms of the faster desynchronized oscillator, $\theta_{\text {fast }}=\theta+\Phi$, can be increased to the maximum shift (i.e., antiphase clusters). The phase separation of two oscillators with natural frequency differences $\Delta \omega=$ $\omega_{\text {fast }}-\omega$ in relation to the Lyapunov exponent, $\Lambda$, of a single negative PRC perturbation,

$\rho=\frac{1}{T} \int_{0}^{T}\left(Z_{n e g}(\theta) I_{n e g}(t)\right) d t$, over one period, $T$, can be found from the following equation [26]:

$$
\dot{\Phi}=\Delta \omega+\frac{1}{T} \int_{0}^{T}\left(Z_{\text {neg }}(\theta+\Phi) I_{\text {neg }}(t)\right) d t
$$

Using a Taylor expansion and truncating, yields:

$$
\begin{aligned}
& \dot{\Phi}=\Delta \omega+\frac{1}{T} \int_{0}^{T}\left(Z_{\text {neg }}(\theta) I_{\text {neg }}(t)\right) d t+\frac{1}{T} \int_{0}^{T}\left(Z^{\prime}{ }_{n e g}(\theta) I_{\text {neg }}(t)\right) d t \Phi+\mathcal{O}\left(\Phi^{2}\right) \\
& \Phi(t)=\frac{\Delta \omega+\rho}{\Lambda}\left(e^{\Lambda \mathrm{t}}-1\right)
\end{aligned}
$$

\subsubsection{Mean Signal}

The mean signal gives a qualitative measurement of phase coherence and can be obtained by averaging individual oscillator intensities at a given time. A non-synchronized state is 
characterized by a noisy mean signal, as illustrated by the black plot in Figure 3.2a. The mean signal in Figure 3.2b represents a partial synchronized state when the coupling is not adequate to show a complete synchronization state. Figure 3.2c shows a regular mean signal over time that exhibits a fully synchronized state in both phase and frequency. The mean signal allows for visualization of the antiphase state, which makes it a complementary tool with quantitative measurements.

a)

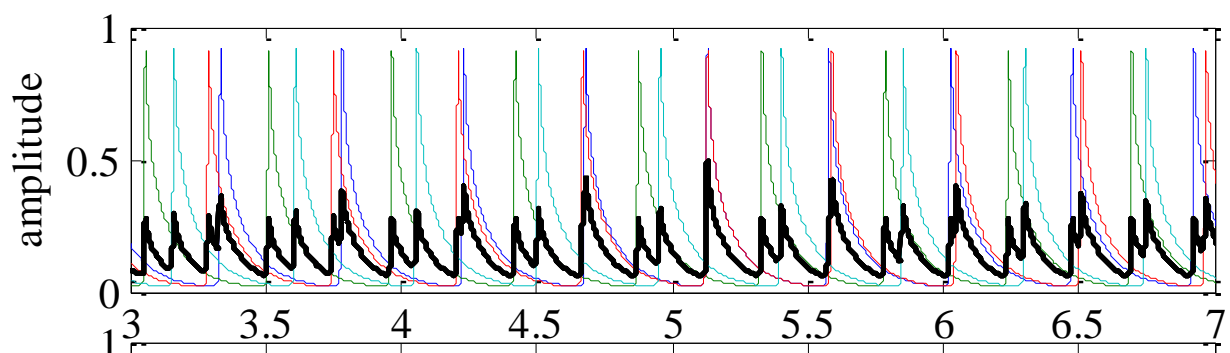

b)

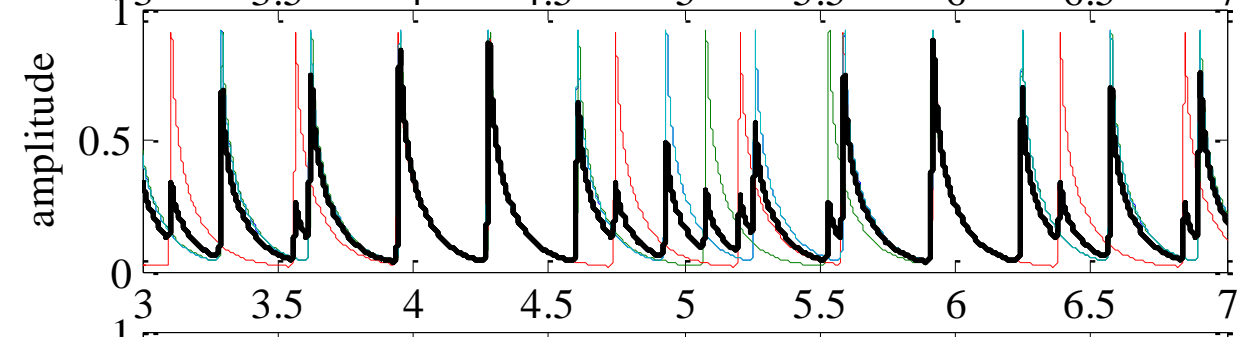

c)

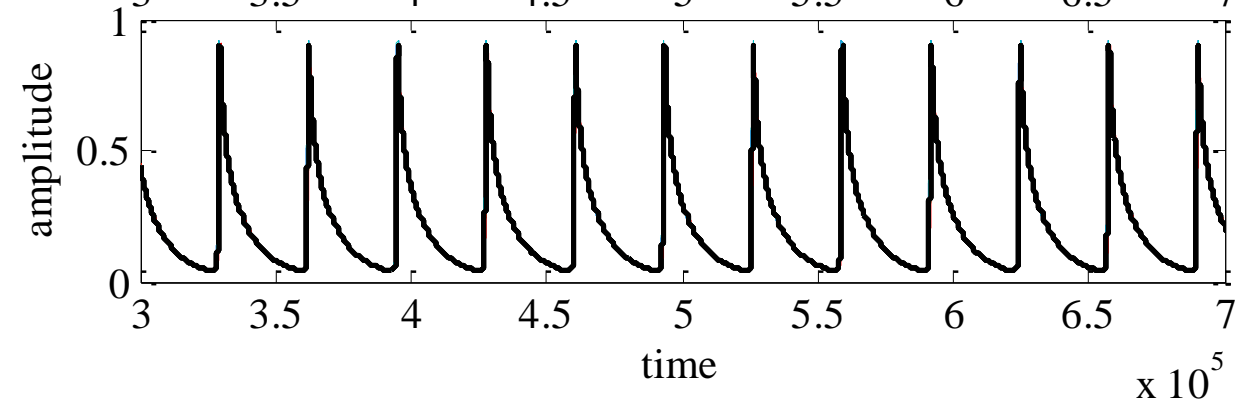

FIG. 3.2: Mean signals. a) A time series showing a noisy mean signal (black) on top of four individual oscillators with non-coherent phases. b) A mean signal (black) of four individual oscillators that are partially synchronized and showing some degree of phase coherence. c) A mean signal (black) of four individual oscillators that are fully synchronized, showing phase coherence. 


\subsubsection{Order Parameter}

The Kuramoto order parameter $r$ is a quantitative measurement of phase coherence. The order parameter ranges from 0 (no synchronization) to 1 (perfect synchronization), which can be calculated from Eq. 1.2 in Section 1.4.1 of Chapter 1. It is typically presented with $r$ as the yaxis, as shown previously in Figure 1.7. Here, the order parameter values are plotted as a function of time to show the sum of the phases at each time point. Based on the phase of each oscillator, a non-coherent state leads to a lower value of the order parameter. The order parameter of a fully synchronized state approaches 1 , whereas that of a partially synchronized state typically fluctuates in mid-range, as illustrated in Figure 3.3. The order parameter alone cannot distinguish an antiphase case from non-coherent phases, because the phases cancel each other in both cases. If an antiphase state occurs in a system, the order parameter will be near zero, which deceptively indicates a non-coherent state. In this case, the mean signal will remove the misperception, as shown in Figure 3.4. 


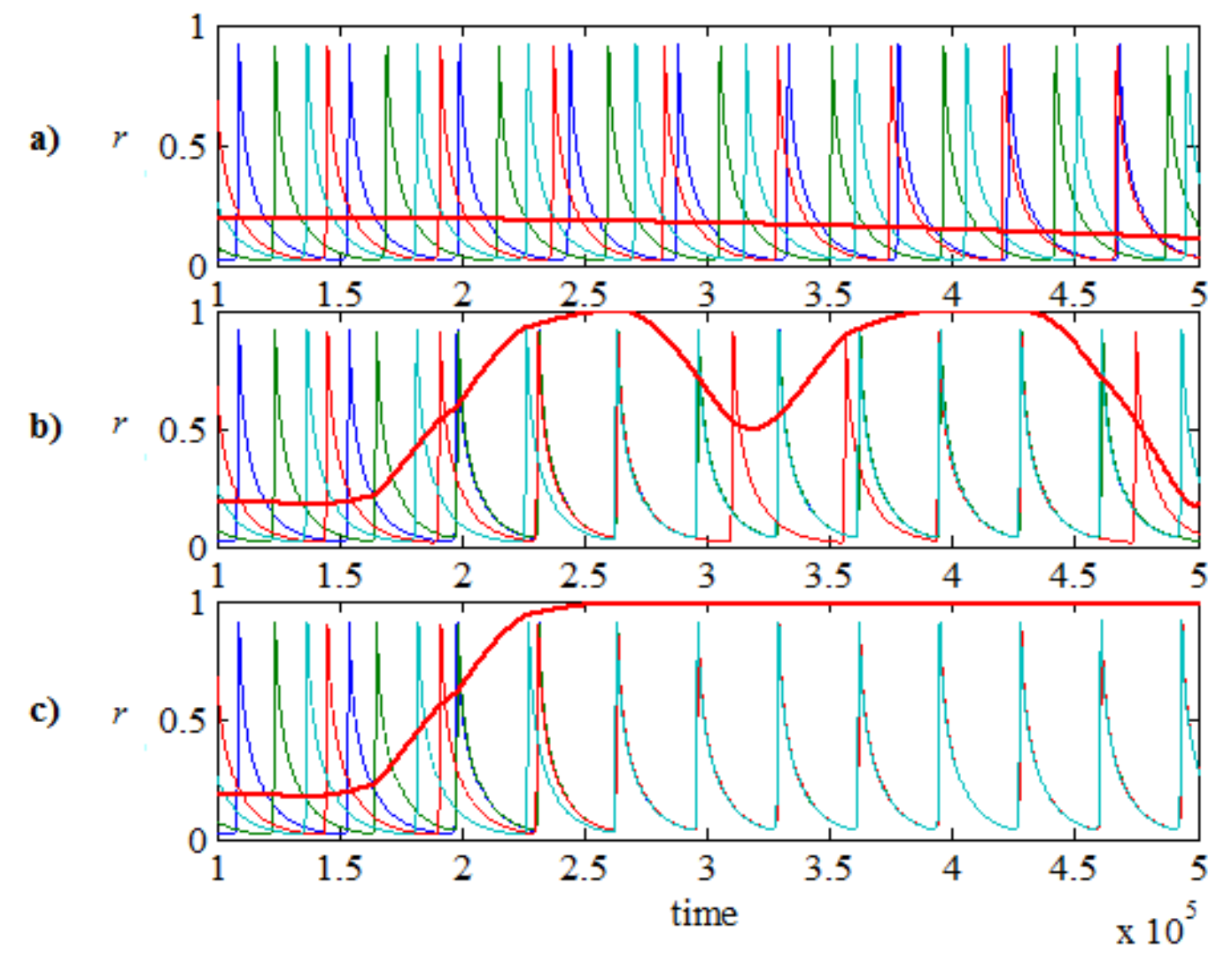

FIG. 3.3: Order parameter $r$ indicating the degree of a phase coherence. a) Order parameter (red) near 0 indicates little coherence in the phases. b) Fluctuations of the order parameter (red) to higher values indicate a partially synchronized state. c) Order parameter (red) of 1 indicates complete synchronization. 


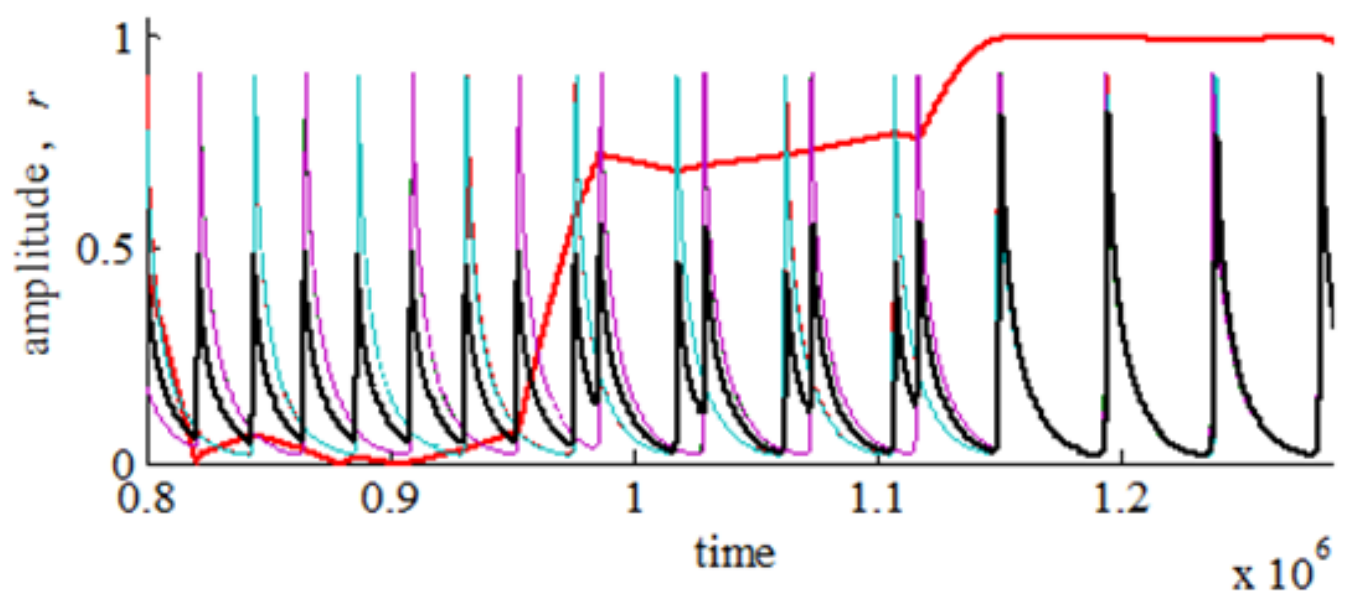

FIG. 3.4: Mean intensity (black) and the order parameter (red) from antiphase synchronization to complete in phase synchronization. The system shows the evolution from antiphase synchronization to partial synchronization to in phase synchronization. The order parameter alone cannot distinguish the antiphase case from the non-coherent case.

\subsection{Experimental Design}

Two experimental designs were used for the creation of a signal synchronized by external periodic perturbations. Experiments were performed using 35 uncoupled photosensitive BZ oscillators. Five oscillators were used as a control for the applied signals based on their changes in natural frequency. The particles were prepared, immersed in catalyst-free BZ solution, and monitored by an imaging system, with the same recipe and instrumentation as described in Section 2.2.1 in Chapter 2.

\subsubsection{Synchronization Experiments}

Periodic positive pulse signals were used for synchronizing uncoupled heterogeneous BZ oscillators. Initially, 30 oscillators oscillate with their natural frequencies for $126.0 \mathrm{~s}$, as shown in 
Figure 3.5a. The natural periods are measured and displayed in the histogram shown in Figure 3.5b. The periodic positive pulses were then introduced at $t=126.0$ s. Figure 3.6 shows projected light intensities that were applied as a repeated positive pulse forcing above the baseline light

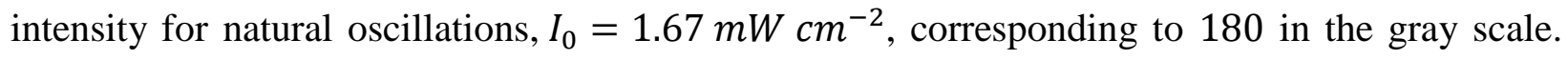
The light intensity for a pulse was set to $I=2.22 \mathrm{~mW} \mathrm{~cm}^{-2}$, corresponding to 240 on the gray scale. Each positive pulse of a fixed amplitude lasted for $9.0 \mathrm{~s}$, after which the background intensity of $I_{0}$ was restored. The positive pulse was applied with an adaptive frequency, which was greater than the mean oscillator frequency. These pulses were periodic, with adaptive periods $9.0 \mathrm{~s}$ less than the mean period of five randomly selected control beads, which corresponds to the time for three image frames in the experiment. The five control oscillators were maintained at their natural frequency at a constant light intensity during the experiment. The mean natural period of the five control beads was measured and the forcing signal was adjusted during the experiment. The forcing signal is an adaptive signal in order to compensate for the natural drift in the oscillator frequency. Figure 3.7 shows the adaptive period of the applied positive pulses to a representative control bead. 

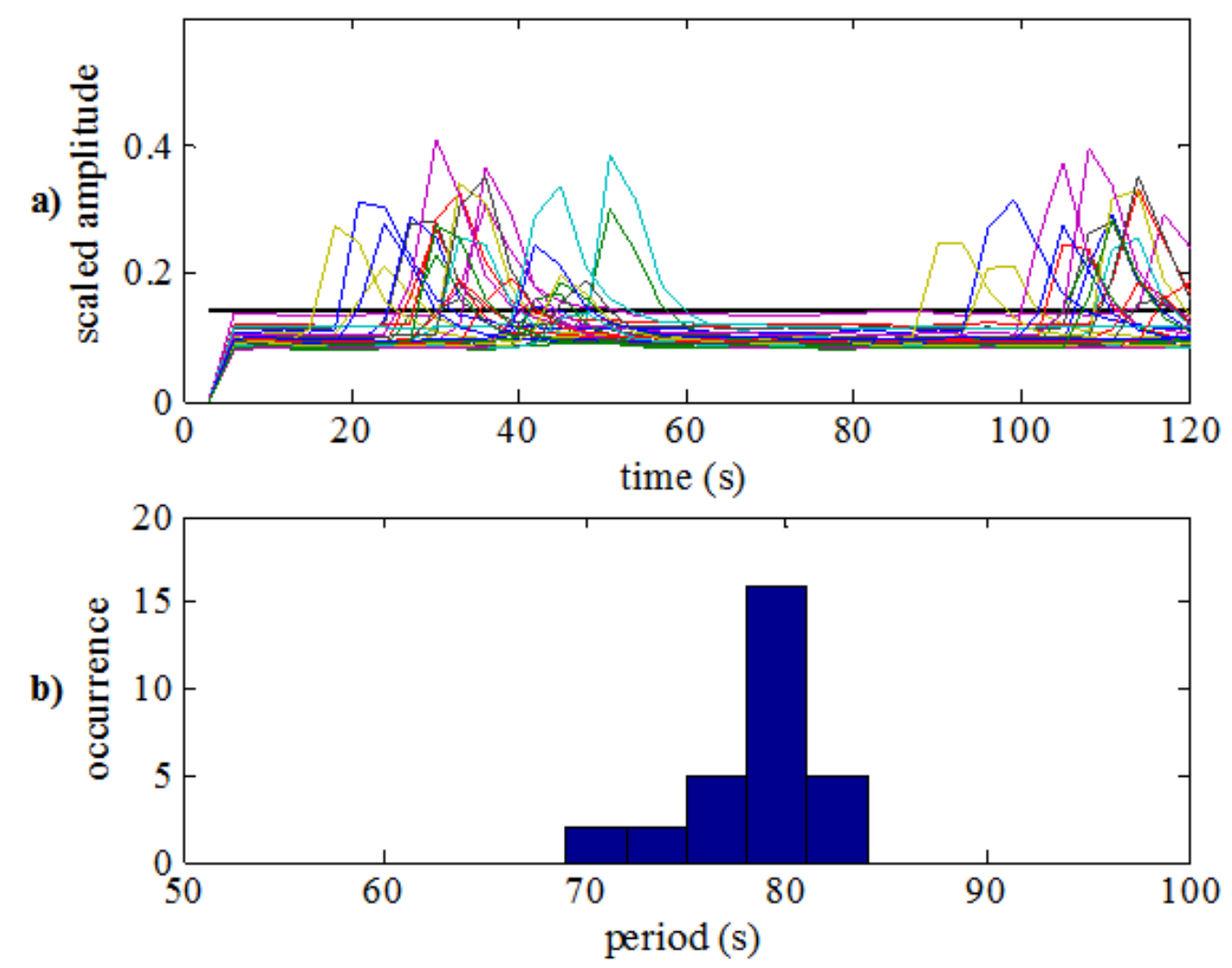

FIG. 3.5: Oscillators at their natural frequency with no external signal. a) The individual time series of 30 oscillators showing their natural oscillations. b) A histogram showing the distribution of the natural periods of 30 oscillators.

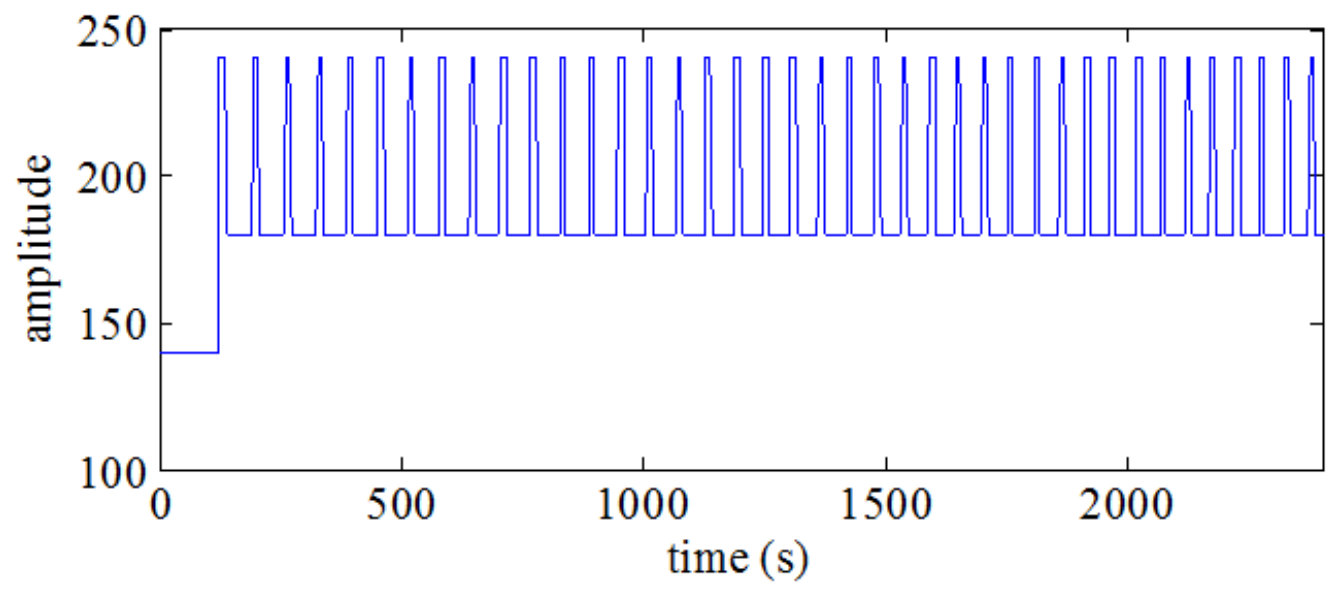

FIG. 3.6: Periodic positive pulses with amplitude $I=0.56 \mathrm{~mW} \mathrm{~cm}^{-2}$ above the background intensity. The perturbations are introduced at $\mathrm{t}=126.0 \mathrm{~s}$ and are shown in gray scale. 


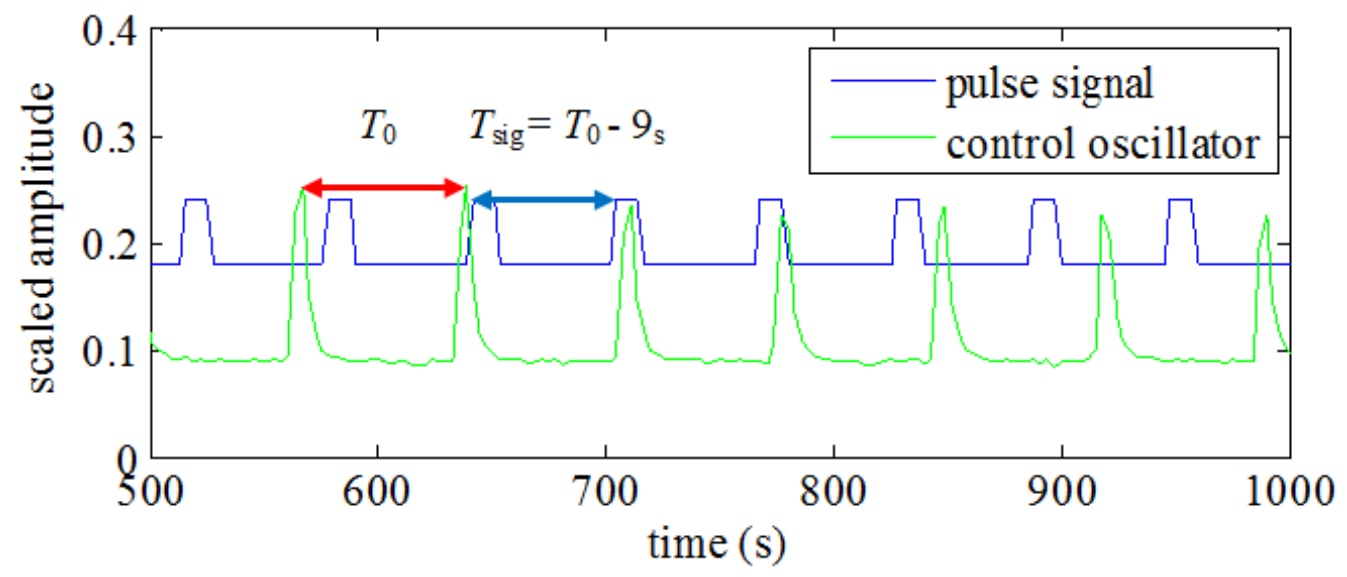

FIG. 3.7: Adaptive periodic positive pulses to the mean periods of five control oscillators. The figure shows one control bead representing the mean natural period of the system, $\mathrm{T}_{0}$. The pulse signals are applied with the adaptive period $\mathrm{T}_{\text {sig }}=\mathrm{T}_{0}-9.0 \mathrm{~s}$.

\subsubsection{Desynchronization Experiments}

After establishing synchronized signals, periodic negative pulse perturbations were introduced at $\mathrm{t}=1200.0 \mathrm{~s}$ by setting the background intensity to its lowest value on the gray scale, as shown in Figure 3.8. The perturbation is applied immediately after the end of the synchronizing positive pulse signal and lasts for the first half of the period. The timing of each negative perturbation was applied in a controlled manner for consecutive cycles. The negative perturbation was based on the PRC, in which a positive slope appeared in the first half of the phase cycle. 


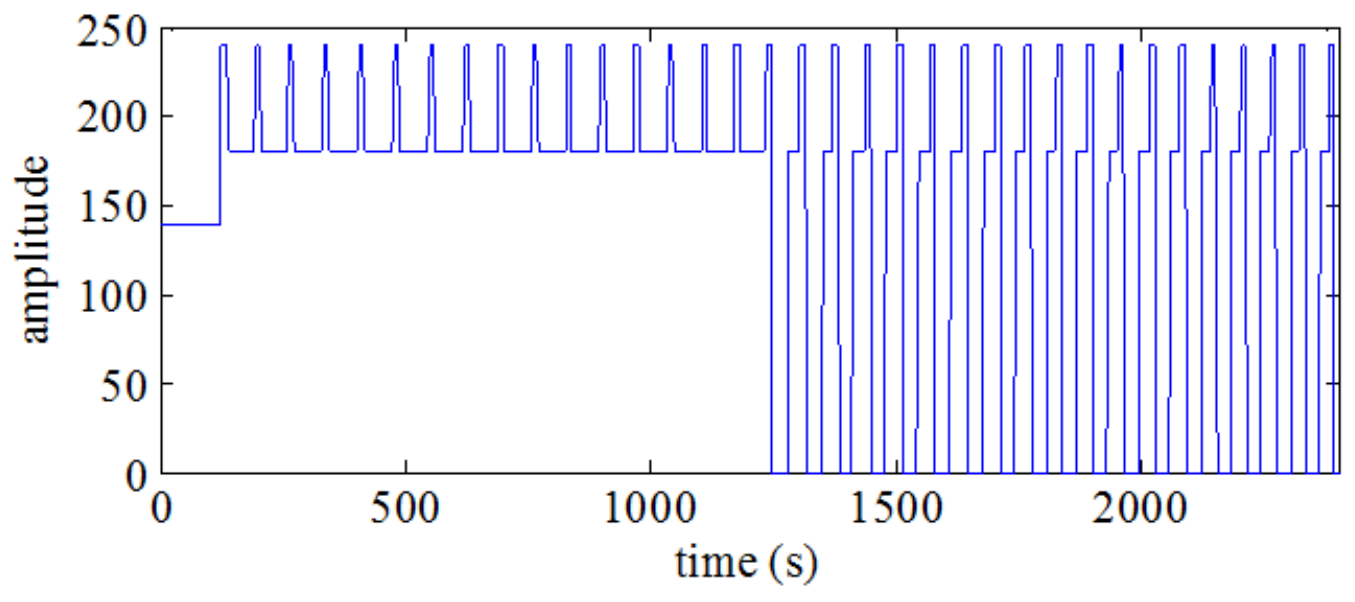

FIG. 3.8: Periodic negative pulses with amplitude $I=1.67 \mathrm{~mW} \mathrm{~cm} \mathrm{~cm}^{-2}$ below the background intensity introduced at $\mathrm{t}=1200.0 \mathrm{~s}$ after the positive pulses, $I=0.56 \mathrm{~mW} \mathrm{~cm}^{-2}$, above the background intensity.

\subsection{Experimental Results}

\subsubsection{Synchronization}

The application of periodic positive pulses results in the entrainment of uncoupled BZ oscillators with a frequency that is equal to the driving frequency of external forcing. Figure 3.9a shows an enhanced mean signal in the black plot, where peaks occurred after $6.0 \mathrm{~s}$ from the forced signal in the blue plot. The mean signal was entrained to the period of the external positive pulses, showing frequency and phase locking. Figure $3.9 \mathrm{~b}$ shows a representative synchronized time series from ten individual oscillators, while Figure 3.9c shows the order parameter with a mean of $r_{\text {mean }}=0.91$, indicating a high degree of synchronization. 


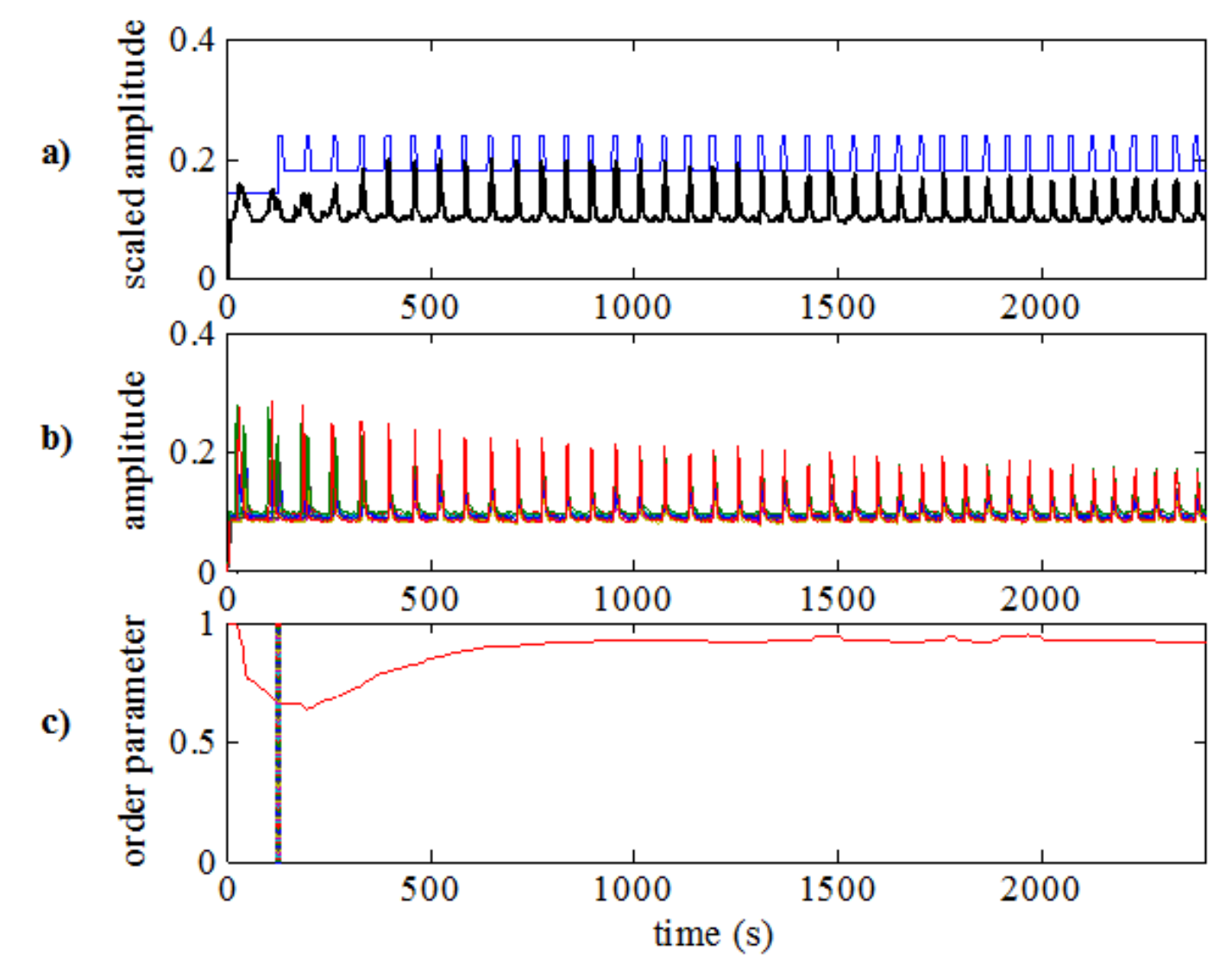

FIG. 3.9: Signal synchronized to external periodic positive pulses in 30 oscillators. a) Positive light pulse (blue) introduced at $\mathrm{t}=126.0 \mathrm{~s}$, with a period faster than the mean period of five control oscillators by 9.0 $\mathrm{s}$ with a width of $9.0 \mathrm{~s}$. The mean signal (black) is regular and synchronized to the period of the positive pulse. b) Individual time series of ten representative oscillators, which show high-phase coherence. c) The order parameter (red) is close to 1.0, indicating a high degree of synchronization. System of 30 microoscillators loaded with $\mathrm{Ru}(\mathrm{bpy})_{3}^{2+}$ catalyst, with a mean natural period of 79.9 s. The background intensity is $I_{0}=1.67 \mathrm{~mW} \mathrm{~cm}^{-2}$. BZ reaction mixture composition: malonic acid, $[M A]=0.08 \mathrm{M}$; sodium bromide, $[\mathrm{NaBr}]=0.02 \mathrm{M}$; sulfuric acid, $\left[\mathrm{H}_{2} \mathrm{SO}_{4}\right]=0.78 \mathrm{M}$; sodium bromate, $\left[\mathrm{NaBrO}_{3}\right]=$ $0.48 \mathrm{M}$.

Discrete BZ oscillators that are synchronized to external pulses resemble the rhythmic patterns of neurons, which are coupled in a pulse-like style manner [30]. Entrainment occurred 
when the frequency of the external pulses was on the same order as the frequency of the oscillators, with a few seconds offset. This has some similarity to the synchronization in 24-h circadian rhythms $[31,32]$. In our system, a frequency forcing signal greater than the mean frequency of the control beads leads to effective entrainment and, subsequently, a fully synchronized state. A similar result was shown by Lin et al. [33] in a photosensitive BZ extended system, where the 1:1 entrainment region (Arnold tongue) was shifted toward higher frequencies of the forcing perturbations while increasing the forcing amplitude. Our results also resemble the entrainment to a higher frequency source shown in experimental studies of BZ extended systems [34]. There have been no experiments previously reported for the synchronization of discrete uncoupled oscillators by external forcing in a BZ system. The entrainment of discrete oscillators resembles entrainment of heart pacemaker cells to external electric signals [23, 35].

\subsubsection{Desynchronization}

Negative pulses are introduced after $\mathrm{t}=1200.0 \mathrm{~s}$. The frequency of negative pulses is equal to the frequency of the positive pulses. PRC analysis suggests that negative pulses should be applied at the first half of the phase cycle to lead to desynchronization, as discussed in Section 3.2.1 and shown in Figure 3.1b. The resulting behavior is shown in Figure 3.10. The mean signal, the individual time series of some representative oscillators, and the order parameter indicate desynchronization as a result of introducing the negative signal into the system. Figure 3.10a shows a reduction in mean signal and cluster formation, indicating the initiation of a desynchronization state. Figure $3.10 \mathrm{~b}$ shows synchronized time series signals for ten oscillators, where some oscillators were pulled out from the original synchronized state. In agreement with the PRC analysis, negative perturbations caused phase advancement of some oscillators with 
desynchronization. Figure 3.10c shows a decreasing order parameter after the introduction of negative pulses at $\mathrm{t}=1200.0 \mathrm{~s}$. The order parameter decreased to $r_{\text {mean }}=0.46$, demonstrating the capability of negative pulses to desynchronize the signal.

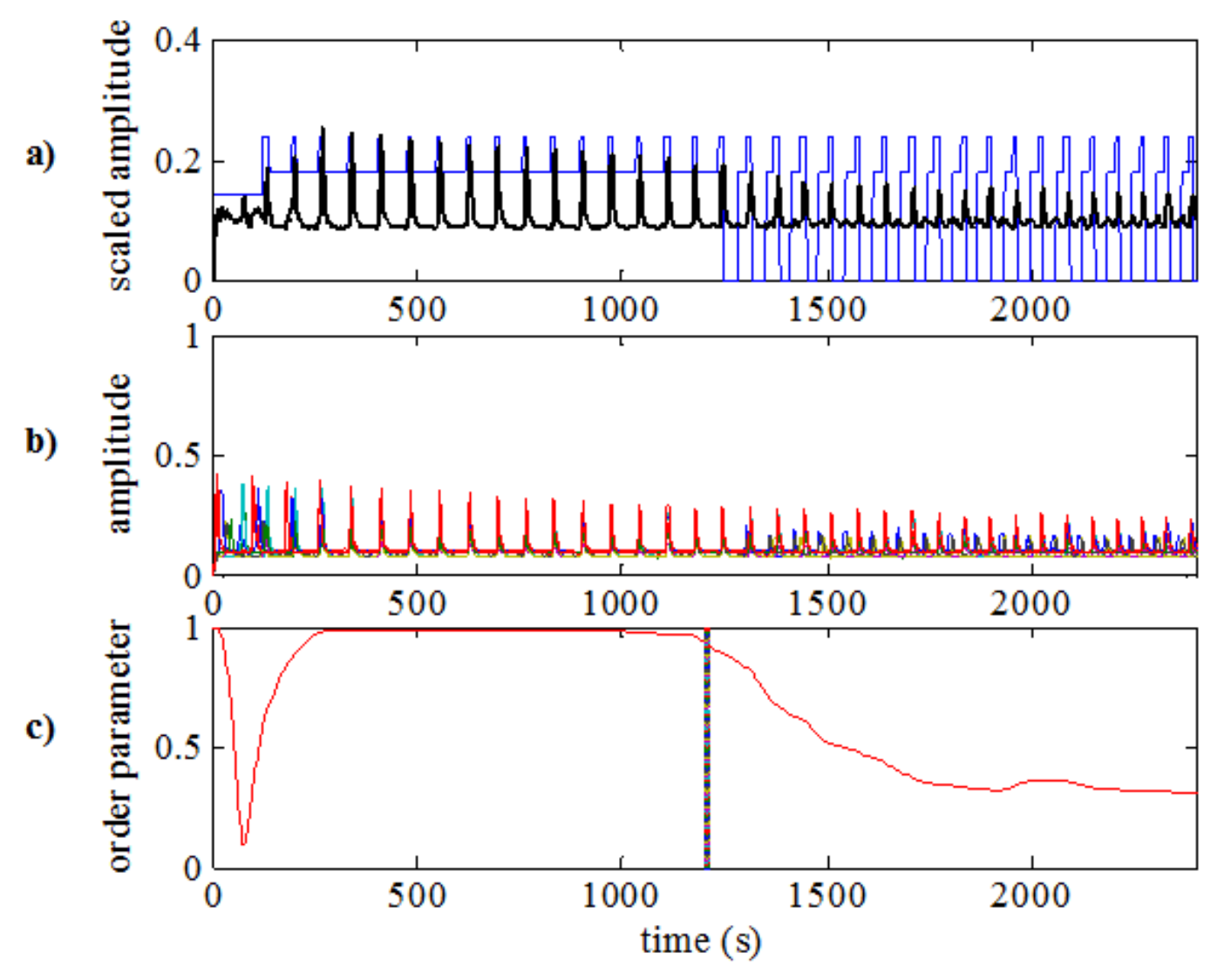

FIG. 3.10: Positive square wave-forcing signal followed by negative pulses during the first half of the period. a) The positive light pulse signal (blue) was introduced at $t=126.0 \mathrm{~s}$, while the negative light pulse signal was introduced at $\mathrm{t}=1200.0 \mathrm{~s}$, immediately after the positive pulses and lasting for the first half of the mean period. The mean signal (black) after $1200.0 \mathrm{~s}$ was attenuated and irregular, indicating desynchronization. b) Individual time series of ten representative oscillators. c) The order parameter (red) is reduced after introducing the negative pulse, indicating desynchronization. System of 30 microoscillators loaded with $\mathrm{Ru}(\mathrm{bpy})_{3}^{2+}$ catalyst, with a mean natural period of $78.7 \mathrm{~s}$. The background intensity is $I_{0}=1.67 \mathrm{~mW} \mathrm{~cm}^{-2}$. The BZ reaction mixture composition is the same as in Figure 3.9. 
The dominant hypothesis for using regular DBS is that it alleviates symptoms by regulating the firing rate of neurons $[13,15,36]$. Our results, however, demonstrate the effectiveness of a periodic pulse stimulus for a desynchronizing mechanism, as proposed in theoretical neuron models [19]. In contrast to the irregular stimulus that induces desynchronization in the Nabi et al. model [18], the stimulus in this study was regular. Moreover, this study applied the stimulus in a defined manner, which mimics the perturbations applied in heart fibrillation, where only perturbations at an early specific phase lead to desynchronization [23].

The applied negative perturbations have been further tested for the last half of the period to confirm that the PRC positive slope is essential for desynchronization. Figure 3.11 shows the result of applying negative pulses at the end of the phase cycle of the synchronized mean signal. The mean signal and individual time series of ten representative oscillators showed that a regular mean signal and a coherent time series were maintained. The order parameter during the experiment was close to 1.0, indicating sustained synchronization after applying negative pulses at the last half of the phase cycle. The calculated mean order parameter was $r_{\text {mean }}=0.94$, which confirms a synchronization state. 
a)

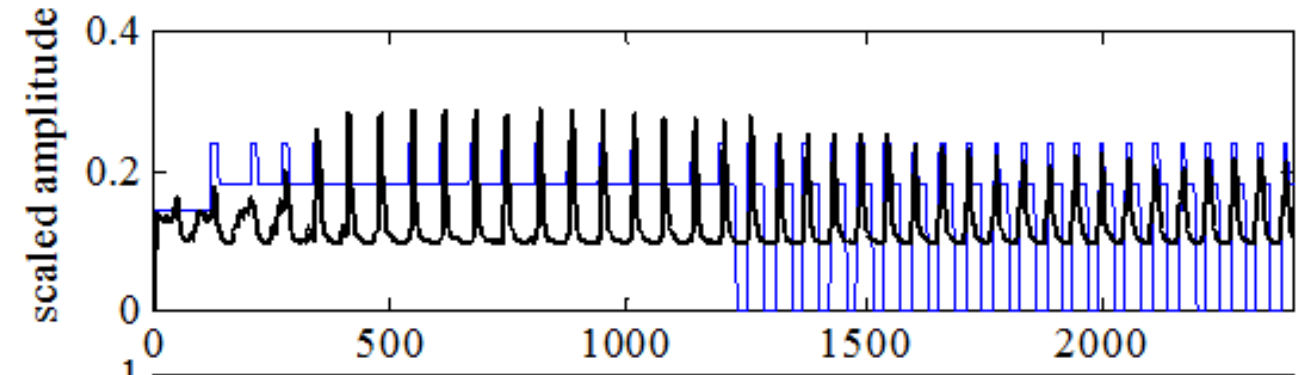

b)

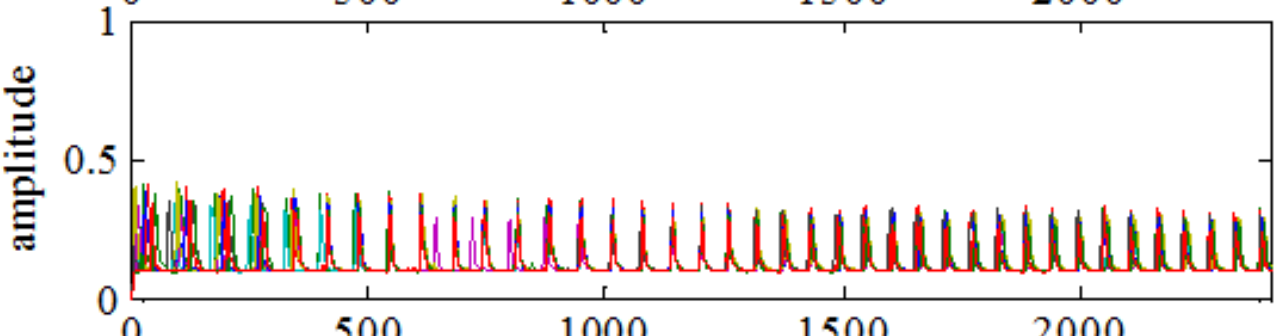

c)

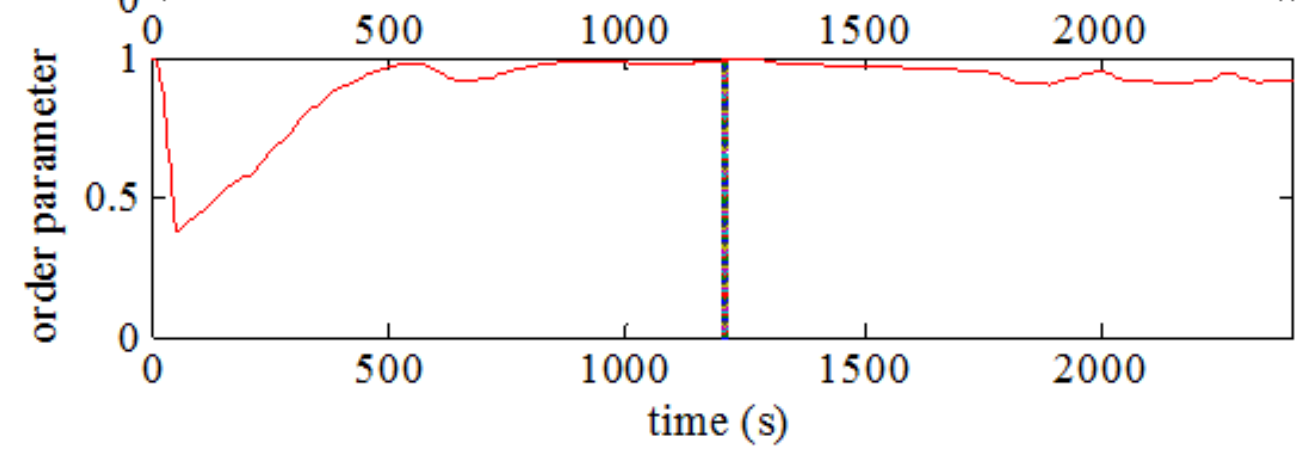

FIG. 3.11: Square wave-forcing signal followed by dark pulses in the last half of the period. a) The positive light pulse signal (blue) is introduced at $t=126.0 \mathrm{~s}$, while the negative light pulse signal is introduced at $\mathrm{t}=1200.0 \mathrm{~s}$, immediately after the positive pulses and lasting for the last half of the mean period. The mean signal (black) after $1200.0 \mathrm{~s}$ preserves regularity, indicating that the negative pulses are not able to desynchronize the oscillators. b) Individual time series of ten representative oscillators. c) The order parameter (red) remains close to 1.0 after introducing the negative pulse, indicating a synchronization state. The system is made up of 30 micro-oscillators loaded with $\mathrm{Ru}(\mathrm{bpy})_{3}^{2+}$ catalyst, with a mean natural period of $83.7 \mathrm{~s}$. The background intensity is $I_{0}=1.67 \mathrm{~mW} \mathrm{~cm}$. The BZ reaction mixture composition is the same as in Figure 3.9. 
Other sets of experiments were conducted by allowing uncoupled oscillators to synchronize with another type of regular signal - the positive half-sine wave signal. The sine wave was obtained according to the following equation:

$$
I=I_{0}+A \sin 2 \pi / T .
$$

In our study, the sine wave of period $T$ had an amplitude of $A=100$, corresponding to the maximum light intensity $I=0.93 \mathrm{~mW} \mathrm{~cm}^{-2}$ above the background intensity $I_{0}=$ $1.29 \mathrm{~mW} \mathrm{~cm}^{-2}$, which corresponded to 140 in the gray scale. The applied positive half-sine wave was introduced at $\mathrm{t}=126.0 \mathrm{~s}$, which has a shorter period than the mean control bead period by $9.0 \mathrm{~s}$. Synchronization of the system was achieved with the positive signal for the first 1200.0 $\mathrm{s}$; it was then forced by periodic negative pulse perturbations for the rest of the duration of the experiment, as shown in Figure 3.12.

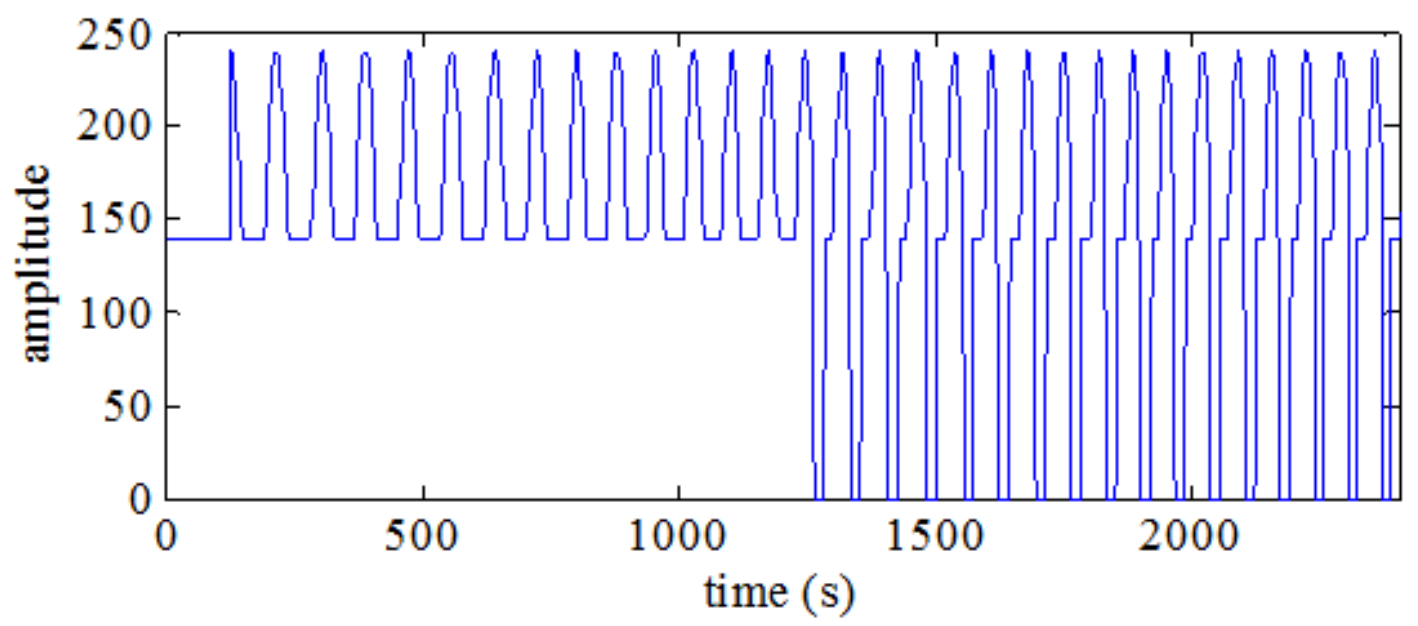

FIG. 3.12: Periodic positive half-sine wave with amplitude of 100 on the gray scale, corresponding to $0.93 \mathrm{~mW} \mathrm{~cm}^{-2}$ above the background intensity, was introduced at $\mathrm{t}=126.0 \mathrm{~s}$. Periodic negative pulses with light intensity $I=1.29 \mathrm{~mW} \mathrm{~cm} \mathrm{~cm}^{-2}$ below the background intensity were introduced after $1200.0 \mathrm{~s}$. 
The negative pulse signal was applied for the first half of the cycle, initiated by a $3.0 \mathrm{~s}$ offset before the end of the positive half-sine wave signal. This offset removed part of the sine wave. This was acceptable because only the first part of the half-sine wave was responsible for entrainment. Our experiments show that the peak of the mean signal in the synchronized region corresponds to the maximum of a $\pm 3.0 \mathrm{~s}$ half-sine wave signal. Therefore, applying negative pulses at the latter half of the half-sine wave signal will exclude the portion of the half sine wave responsible for entrainment. Applying a negative pulse at the latter half of the mean cycle signal was not possible because it would remove the earlier portion of the half sine wave responsible for entrainment.

Figure 3.13 shows behavior resulting from applying negative pulses at the first half of the cycle of the mean signal. The degree of desynchronization shown via the mean signal, the individual time series, and the order parameter was less than what was observed with the squarewave forcing system. The amount of desynchronization was measured by the mean order parameter, $r_{\text {mean }}=0.80$, which was greater than in the previous external positive and negative pulse desynchronization experiment shown in Figure 3.10c. 


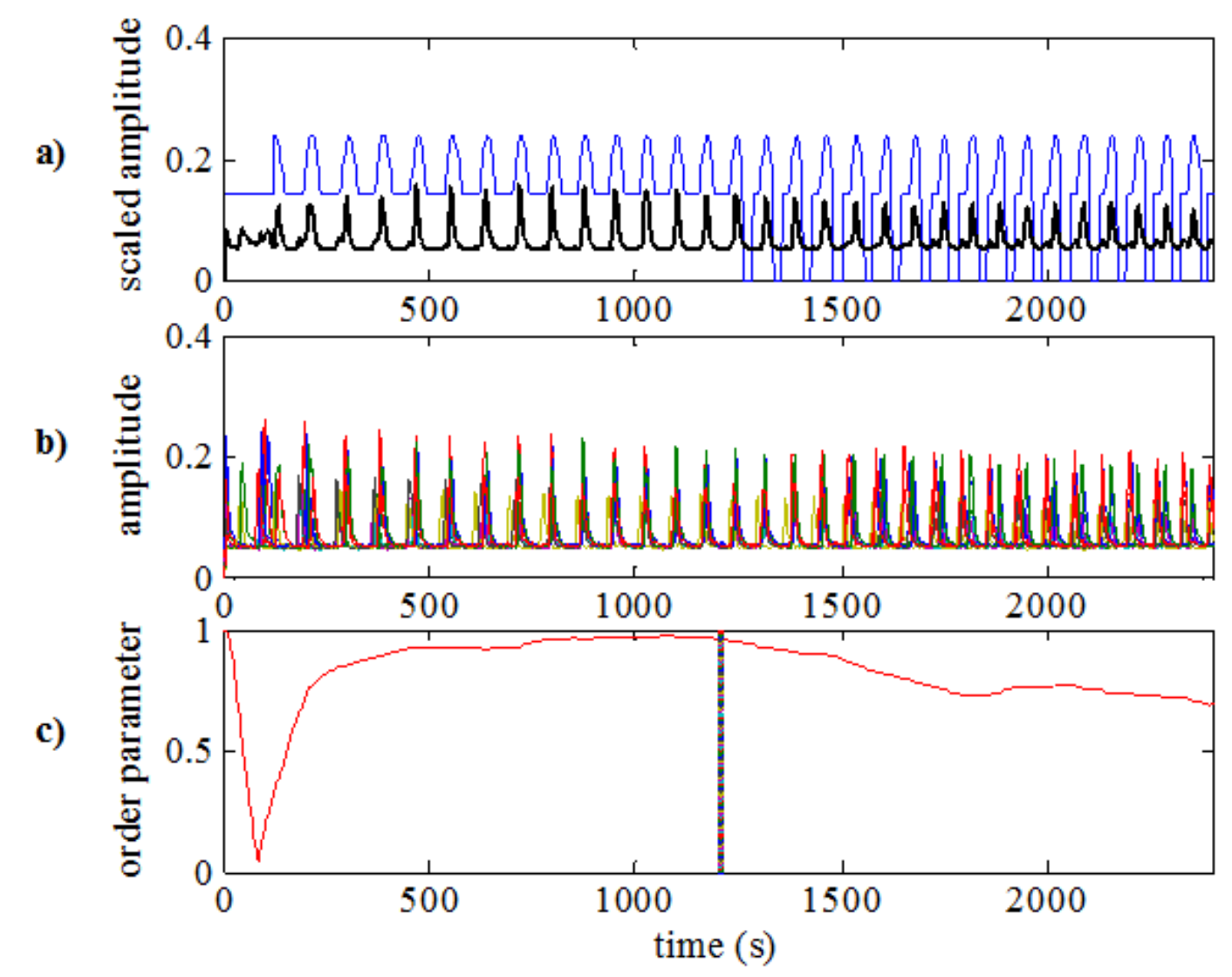

FIG. 3.13: Half-sine wave forcing signal followed by negative pulses applied for the first half of the mean period with a $3.0 \mathrm{~s}$ offset from the half-sine wave signal. a) The positive half-sine wave light signal (blue) is introduced at $\mathrm{t}=126.0 \mathrm{~s}$, while the negative light pulse signal is introduced at $\mathrm{t}=1200.0 \mathrm{~s}$, with a $3.0 \mathrm{~s}$ offset from the end of the half-sine wave signal that lasted for the first half of the mean period. The mean signal (black) is slightly reduced after $1200.0 \mathrm{~s}$, indicating some desynchronization. b) Individual time series of ten representative oscillators, showing some loss of phase coherence among the oscillators. c) The order parameter (red) is slightly reduced after introducing the negative pulse, indicating some extent of desynchronization. The system is made up of 30 micro-oscillators loaded with $\mathrm{Ru}(\mathrm{bpy})_{3}^{2+}$ catalyst, with a mean natural period of $90.1 \mathrm{~s}$. The background intensity is $I_{0}=1.29 \mathrm{~mW} \mathrm{~cm}^{-2}$. The BZ reaction mixture composition is the same as in Figure 3.9. 
The desynchronization was less pronounced when using a positive half-sine wave signal, because of its broader nature. This required initiating negative pulses at the last portion of the positive half-sine wave signal, which covers the first quarter of the synchronized period. Figure 3.14 shows applied negative pulses with a $6.0 \mathrm{~s}$ offset from the end of the positive half-sine wave signal. After applying negative pulses, the order parameter was reduced from $r_{\text {mean }}=0.72$ of the synchronized region to $r_{\text {mean }}=0.40$, indicating significant desynchronization. The degree of desynchronization was more than when applying negative pulses with only a $3.0 \mathrm{~s}$ offset before the end of the positive sine wave signal. 


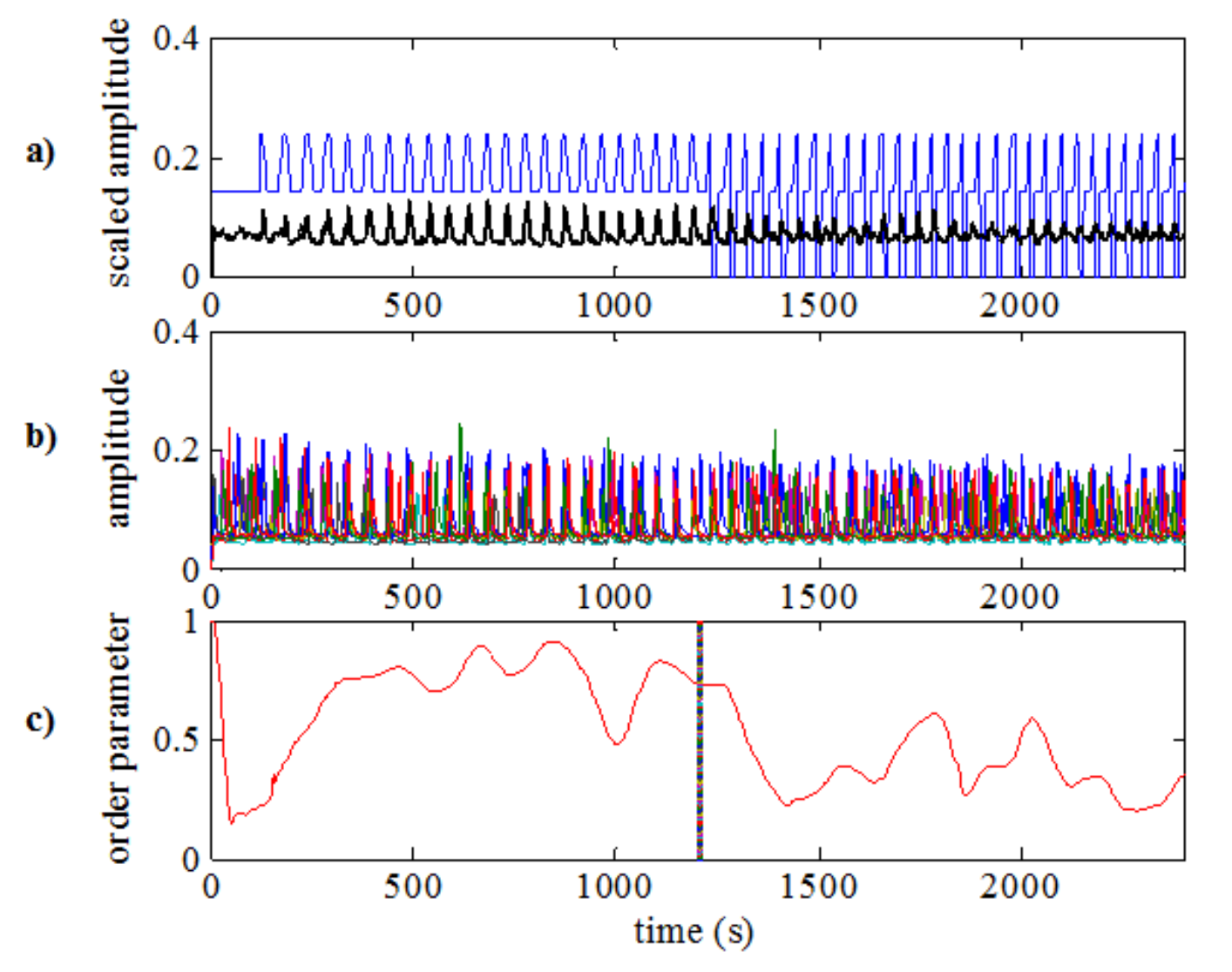

FIG. 3.14: Sine wave forcing signal followed by negative pulses for the first half of the mean period with a $6.0 \mathrm{~s}$ offset from the half-sine wave signal. a) A positive half-sine wave light signal (blue) introduced at $\mathrm{t}=126.0 \mathrm{~s}$, while the negative light pulse signal is introduced at $\mathrm{t}=1200.0 \mathrm{~s}$, with a $3.0 \mathrm{~s}$ offset from the end of the half-sine wave signal that lasted for the first half of the mean period. The mean signal (black) is reduced after 1200.0 s, indicating desynchronization. b) Individual time series of ten representative oscillators show loss of phase coherence among oscillators. c) The order parameter (red) is reduced after introducing the negative pulse, indicating desynchronization. System of 30 micro-oscillators loaded with $\mathrm{Ru}(\mathrm{bpy})_{3}^{2+}$ catalyst, with a mean natural period of $62.4 \mathrm{~s}$. The background intensity is $I_{0}=1.29 \mathrm{~mW} \mathrm{~cm}^{-2}$. The BZ reaction mixture composition is the same as in Figure 3.9. 


\subsection{Simulation}

\subsubsection{Model}

The pulse forcing experiments were modeled with the dimensionless two-variable ZBKE model for the photosensitive BZ reaction [37], as follows:

$$
\begin{aligned}
& d x / d \tau=\varphi_{\text {pos }}+\varphi_{\text {neg }}+f(x, z), \\
& d z / d \tau=\varphi_{\text {pos }}+\varphi_{\text {neg }}+g(x, z),
\end{aligned}
$$

where $\varphi_{\text {pos }}$ and $\varphi_{\text {neg }}$ describes forcing positive and negative light signals. The chemical rates of the system are given by the functions $f$ and $g$, where $x$ and $z$ are the concentrations of $\mathrm{HBrO}_{2}$ and $\mathrm{Ru}(\mathrm{bpy})_{3}^{3+}$, respectively. The simulation was performed with eight oscillators for $1,000,000$ time steps. The Euler method was used in the simulations, where $\Delta t=0.0008$. The frequency heterogeneity of the eight oscillators is defined by the period variation of $35.4 \pm 1.7$. The system was allowed to synchronize from a random initial phase distribution by periodic forcing with a positive light pulse, with the maximum value $\varphi_{p o s}=1 \times 10^{-3}$. Negative pulses of a maximum $\varphi_{n e g}=7 \times 10^{-3}$ were then applied at different times of the applied positive signal. Two different timings of the negative perturbations were carried out for a comparison of the simulations and experiments, with one at the beginning and the other at the end of the phase cycle.

\subsubsection{Simulation Results}

The simulation results qualitatively agree with the experimental results. Figure 3.15 shows the simulation results of applying negative pulses at the beginning of the phase cycle. Figure 
3.15a shows enhancement of the mean signal in the black plot, in the first 521,000 steps, which coincides with the period of external positive pulses $(32,800$ time steps) in the blue plot. This shows the initial condition of phase locking prior to desynchronization. After $t=521,000$ steps, a negative pulse was introduced after each positive pulse at the beginning of the cycle. Figure 3.15a shows that the mean signal collapses into a noisy signal, indicating desynchronization. Figure 3.15b shows individual time series signals for the eight oscillators, with loss of phase coherence after introducing the negative pulses. Figure 3.15c shows a decrease in the order parameter from $r_{\text {mean }}=1.0$ for the fully synchronized state to $r_{\text {mean }}=0.48$, which confirms that the negative pulses desynchronize the oscillators. 
a)

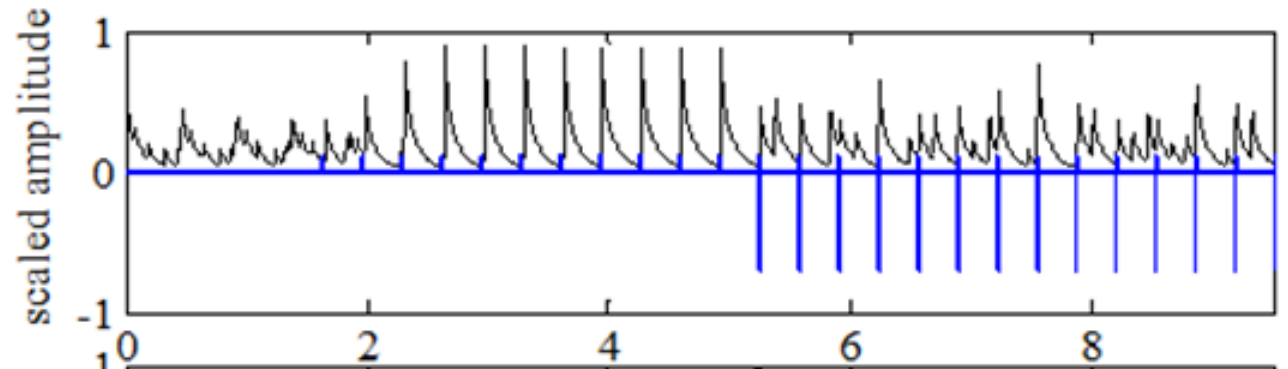

b)

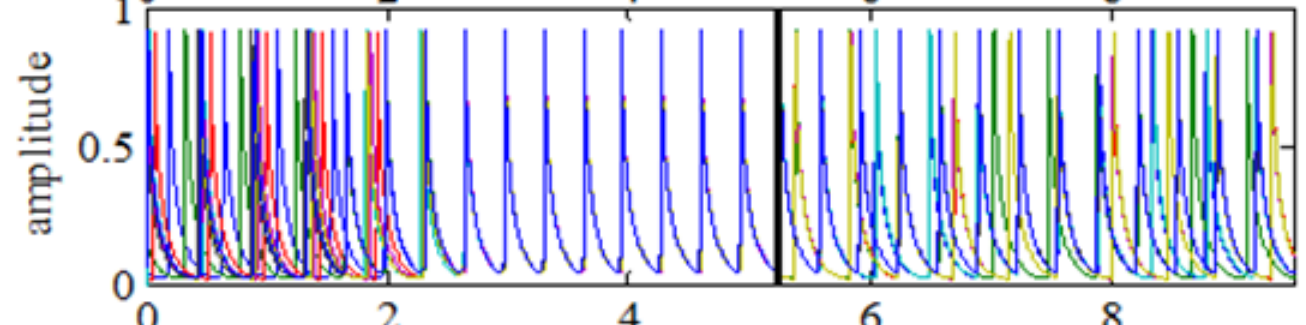

c)

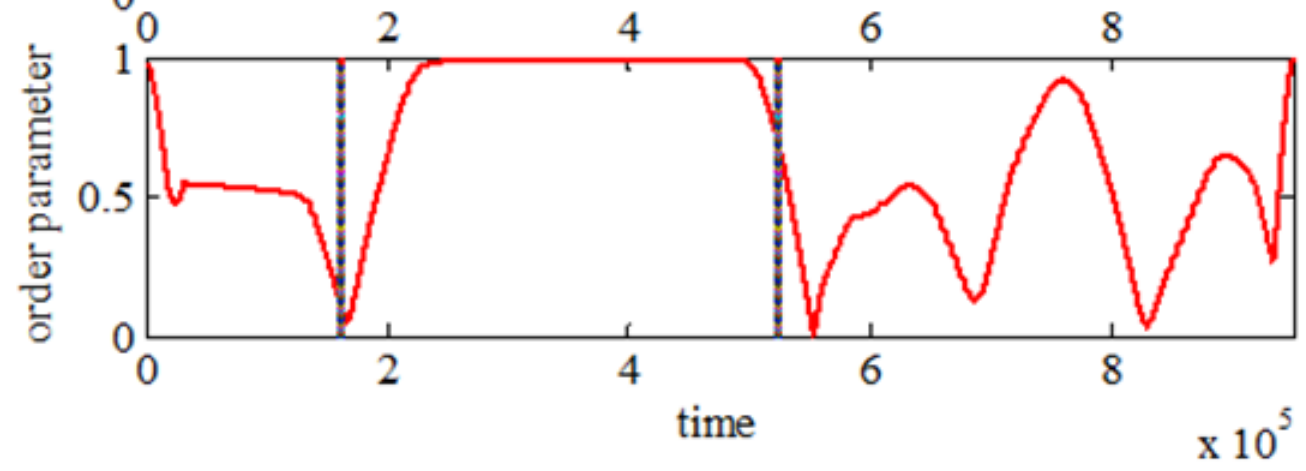

FIG. 3.15: Positive square-wave forcing signal followed by negative pulses applied from the beginning of the phase cycle of the synchronized mean signal of eight oscillators. a) The positive light pulse signal (blue) is introduced at $\mathrm{t}=160000$ time steps, while the negative light pulse signal is introduced at $\mathrm{t}=521$ 000 time steps immediately after the positive pulses. The mean signal (black) decreases and becomes irregular after 521000 time steps, indicating desynchronization. b) Individual time series of the eight oscillators shows phase incoherence. c) The order parameter (red) is reduced after introducing the negative pulse, indicating desynchronization.

The system was further tested by introducing negative perturbations at the end of the cycle. Figure 3.16 shows simulation results from applying negative pulses at the end of the phase cycle. The mean signal and time series of eight oscillators showed that the synchronized behavior remained after negative perturbations were applied. The order parameter also demonstrates 
sustained synchronization after applying negative pulses at the end of the phase cycle. The calculated mean order parameter was maintained at $r_{\text {mean }}=1$, representing a fully synchronized state.

a)
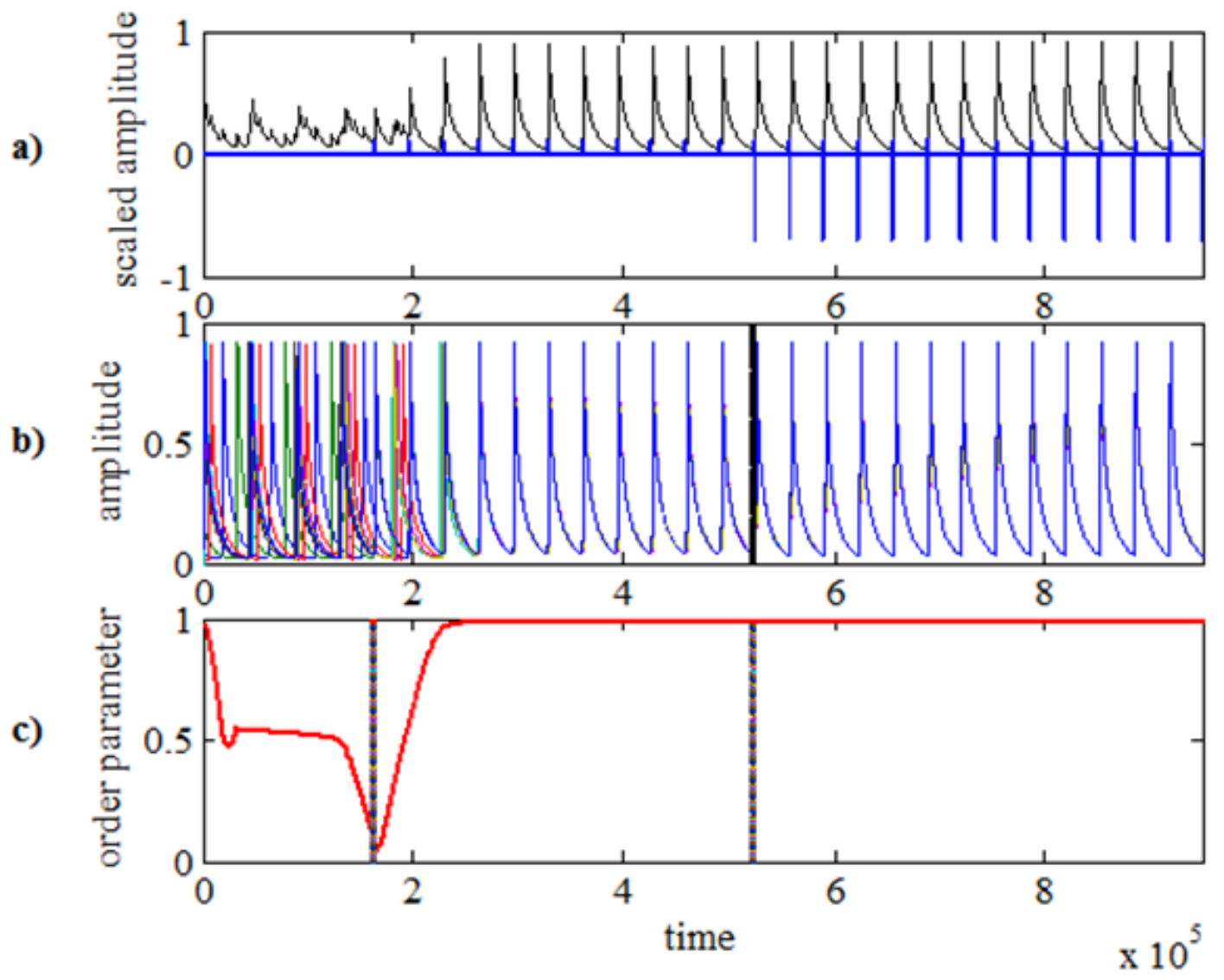

FIG. 3.16: Positive square-wave forcing signal followed by negative pulses applied at the end of the phase cycle of the synchronized mean signal of eight oscillators. a) The positive light pulse signal (blue) is introduced at $\mathrm{t}=160000$ time steps, while the negative light pulse signal is introduced at $\mathrm{t}=521000$ time steps, before the positive pulses at the end of the phase cycle. The mean signal (black) preserved its regularity after 521000 time steps, indicating that these negative pulses are not able to desynchronize the signal. b) Individual time series of the eight oscillators maintains their phase coherence. c) The order parameter (red) continues to be one after introducing the negative pulse, indicating maintenance of the synchronized signal. 
The optimal position for applying negative perturbation was determined by the sign of the Lyapunov exponent expressed by the slope of PRC [19]. The simulation results demonstrated that the PRC slope is essential in the desynchronization with regular negative pulse perturbations. This implies the necessity of determining the cycle phase in a known synchronized state to ensure that the timing of perturbations is applied during the beginning of the phase cycle for effective desynchronization.

\subsection{Summary}

This study was carried out by establishing a synchronized signal with positive perturbations and then forcing it with appropriate negative perturbations to cause desynchronization. The timing of negative pulse perturbations was based on a PRC analysis and the sign of the Lyapunov exponent. Both simulation and experimental results confirm that application of negative perturbations at phases of the positive slope region of the PRC can lead to desynchronization. Information about synchronization/desynchronization was displayed via mean signals, individual time series, and order parameter calculations. These results support theoretical desynchronization mechanisms of DBS over entrainment mechanisms [10-12, 16]. The theoretical method reported by Wilson and Moehlis [19], based only on PRC information, was experimentally verified in this study using the BZ system. This method can be introduced to desynchronize abnormal synchronous oscillations in the basal ganglia, which are thought to correlate to tremors in PD [6-8]. Further studies should include closed-loop feedback to update information during desynchronization in the BZ system. This step may be important in designing a new generation of a control DBS to replace the currently available permanent DBS [25, 26]. The closed-loop system has the advantage of applying therapy only as needed with less tissue damage and more energy efficiency. In this study, we used a quasi-closed-loop system, where 
oscillators' phases were synchronized with an adaptive positive stimulus prior to administration of a regular desynchronizing stimulus. This allowed for information about the synchronized mean signal to be obtained, which aided in the application of negative pulses in consecutive cycles. 


\section{Reference}

[1] R. Melzack and P. D. Wall, "Pain mechanisms: A new theory." Science 150, 971-979 (1965).

Online Version

[2] R. Melzack, "Pain - an Overview." Acta Anaesthesiol. Scand. 43, 880-884 (1999). Online Version

[3] P. Brown and C. D. Marsden, "What do the basal ganglia do?" Lancet 351, 1801-1804 (1998).

Online Version

[4] P. J. Uhlhaas and W. Singer, "Neural synchrony in brain disorders: Relevance for cognitive dysfunctions and pathophysiology.” Neuron 52, 155-168 (2006).

Online Version

[5] C. Hammond, H. Bergman, and P. Brown, "Pathological synchronization in Parkinson's disease: Networks, models and treatments.” Trends Neurosci. 30, 357-364 (2007).

Online Version

[6] J. Volkmann, M. Joliot, A. Mogilner, A. Ioannides, F. Lado, E. Fazzini, U. Ribary, and R. Llinás, "Central motor loop oscillations in Parkinsonian resting tremor revealed by magnetoencephalography." Neurology 46 1359-1370 (1996).

Online Version

[7] H. Bergman, A. Raz, A. Feingold, A. Nini, I. Nelken, B-P. Hilla, D. Hansel, and A. Reches, "Physiology of MPTP tremor.” Mov. Disord. 13, 29-34 (1998).

Online Version 
[8] R. Levy, W. Hutchison, A. Lozano, and J. Dostrovsky, "High-Frequency synchronization of neuronal activity in the subthalamic nucleus of Parkinsonian patients with limb tremor." J. Neurosci. 20, 7766-7775 (2000).

Online Version

[9] C. C. Chen, V. Litvak, T. Gilbertson, A. Kühn, C. Lu, S. Lee, C. Tsai, S. Tisch, P. Limousin, M. Hariz, and P. Brown, "Excessive synchronization of basal ganglia neurons at $20 \mathrm{~Hz}$ slows movement in Parkinson's disease', Exp. Neurol. 205, 214221(2007).

Online Version

[10] A. Nini, A. Feingold, H. Slovin, and H. Bergman, "Neurons in the globus pallidus do not show correlated activity in the normal monkey, but phase-locked oscillations appear in the MPTP model of Parkinsonism.” J. Neurophysiol. 74, 1800-1805 (1995).

Online Version

[11] P. A. Tass, "Desynchronizing double-pulse phase resetting and application to deep brain stimulation.” Biol. Cybern. 85, 343-354 (2001).

Online Version

[12] C. J. Wilson, B. Beverlin, and T. Netoff, "Chaotic desynchronization as the therapeutic mechanism of deep brain stimulation.” Front. Syst. Neurosci. 5, 00050 (2011).

Online Version

[13] E. Montgomery and K. Baker, "Mechanisms of deep brain stimulation and future technical developments.” Neurol. Res. 22, 259-266 (2000).

Online Version 
[14] J. E. Rubin and D. Terman, "High frequency stimulation of the subthalamic nucleus eliminates pathological thalamic rhythmicity in a computational model.” J. Comput. Neurosci. 16, 211-235 (2004).

Online Version

[15] G. C. McConnell, R. So, J. Hilliard, P. Lopomo, and W. Grill, "Effective deep brain stimulation suppresses low-frequency network oscillations in the basal ganglia by regularizing neural firing patterns.” J. Neurosci. 32, 15657-15668 (2012).

Online Version

[16] P. A. Tass and M. Majtanik, "Long-term anti-kindling effects of desynchronizing brain stimulation: A theoretical study." Biol. Cybern. 94, 58-66 (2006).

Online Version

[17] P. A. Tass and C. Hauptmann, "Anti-kindling achieved by stimulation targeting slow synaptic dynamics.” Restor. Neurol. Neurosci. 27 589-609 (2009).

Online Version

[18] A. Nabi, M. Mirzadeh, F. Gibou, and J. Moehlis, "Minimum energy desynchronizing control for coupled neurons.” J. Comput. Neurosci. 34 259-271 (2013).

Online Version

[19] D. Wilson and J. Moehlis, "Optimal chaotic desynchronization for neural populations." SIAM J. Appl. Dyn. Syst. 13, 276-305 (2014).

Online Version

[20] P. Danzl, J. Hespanha, and J. Moehlis. "Event-based minimum-time control of oscillatory neuron models." Biol. Cybern. 101, 387-399 (2009).

Online Version 
[21] O. Popovych, C. Hauptmann, and P. A. Tass. "Control of neuronal synchrony by nonlinear delayed feedback.” Biol. Cybern. 95, 69-85 (2006).

Online Version

[22] I. Z. Kiss, C. G. Rusin, H. Kori, and J. L. Hudson, "Engineering complex dynamical structures: Sequential patterns and desynchronization.” Science 316, 1886-1889 (2007).

Online Version

[23] G. R. Mines. "On circulating excitations in heart muscles and their possible relation to tachycardia and fibrillation.” Trans. R. Soc. Can.8, 43-52 (1914).

Online Version

[24] P. A. Tass, "Desynchronization by means of a coordinated reset of neural subpopulations: A novel technique for demand-controlled deep brain stimulation. Prog. Theor. Phys. Supplement 150, 281-296 (2003).

Online Version

[25] O. Popovych and P. A. Tass, "Desynchronizing electrical and sensory coordinated reset neuromodulation.” Front. Hum. Neurosci. 6, 00058 (2012).

Online Version

[26] D. Wilson and J. Moehlis, “An energy-optimal approach for entrainment of uncertain circadian oscillators.” Biophys. J. 107, 1744-1755 (2014).

Online Version

[27] T. Netoff, M. A. Schwemmer, and T. J. Lewis. "Experimentally estimating phase response curves of neurons: Theoretical and practical issues." In Phase Response 
Curves in Neuroscience, edited by N. Schultheiss, A. Prinz, and R. Butera (Springer, New York, 2012), pp. 95-129.

Online Version

[28] A. Pikovsky, M. Rosenblum, and J. Kurths, Synchronization: A universal concept in nonlinear sciences (Cambridge University Press, Cambridge, 2003).

Online Version

[29] E. Brown , J. Moehlis, and P. Holmes. "On the phase reduction and response dynamics of neural oscillator populations.” Neural Comput. 16, 673-715. (2004).

Online Version

[30] C. C. Canavier and S. Achuthan, "History of the application of the phase resetting curve to neurons coupled in a pulsatile manner." In Phase Response Curves in Neuroscience, edited by N. Schultheiss, A. Prinz, and R. Butera (Springer, New York, 2012), pp. 73-91.

Online Version

[31] K. P. Wright, A. W. McHill, B. R. Birks, B. R. Griffin, T. Rusterholz, and E. D. Chinoy, "Entrainment of the human circadian clock to the natural light-dark cycle." Curr. Biol. 23, 1554-1558 (2013).

Online Version

[32] S. Panda, J. Hogenesch, and S. Kay, "Circadian rhythms from flies to human." Nature 417, 329-335 (2002).

Online Version

[33] A. L. Lin, A. Hagberg, E. Meron, and H. L. Swinney, "Resonance tongues and patterns in periodically forced reaction-diffusion systems.” Phys. Rev. E 69, 066217 (2004). 


\section{Online Version}

[34] O-U. Kheowan, E. Mihaliuk, B. Blasius, I. Sendiña-Nadal, and K. Showalter, "Wave mediated synchronization of nonuniform oscillatory media.” Phys. Rev. Lett. 98, 074101(2007).

Online Version

[35] J. Jalife, V. Slenter, J. Salata, and D. Michaels, "Dynamic vagal control of pacemaker activity in the mammalian sinoatrial node.” Circ. Res. 52, 642-656 (1983).

Online Version

[36] A. D. Dorval, A. Kuncel, M. Birdno, D. Turner, and W. Grill, "Deep brain stimulation alleviates Parkinsonian bradykinesia by regularizing pallidal activity.” J. Neurophysiol. 104, 911-921 (2010).

Online Version

[37] A. F. Taylor, P. Kapetanopoulos, B. Whitaker, R. Toth, L. Bull, and M. R. Tinsley, "Phase clustering in globally coupled photochemical oscillators." Eur. Phys. J. Spec. Top. 165, 137-149 (2008).

Online Version 


\section{Chapter 4}

\section{Desynchronization in Populations of Uncoupled Chemical Oscillators with Colored Noise by Periodic Forcing}

\subsection{Introduction}

Oscillators in natural settings are inevitably influenced by their noisy environment. As generally understood, background noise induces disorder and degrades signal quality. However, in some cases, noise induces order and improves the quality of signal detection. Physiological oscillators typically fluctuate over time in response to changes in their environments [1]. A study in neural information coding suggested that neural rhythms are a result of an intrinsic noise mechanism [2], where the synaptic background inputs resemble white or colored (filtered) noise [3]. In vitro experiments have shown a noise-induced order in spiking time of cortical neurons [2]. Moreover, a study by Fellous et al. [4] showed that synaptic background noise enhances phase-locked spiking of pyramidal cells. Synchronization or rhythmic activities are important in many bodily functions, such as the sleep-wake cycle [5], heart beating [6], and respiration [7]. However, in some cases, synchronization is considered abnormal and leads to diseases, as in the case of tremors in Parkinson's disease [8-12]. Abnormally synchronized neural activities may be attributed to the interference of intrinsic noise acting as an external forcing signal, which synchronizes neural activities or enhances their synchronization process. This hypothesis has support from both theoretical and experimental noise studies. Noise is shown to induce order and coherent patterns, as well as allow amplification and coherence of weak signals, such as stochastic resonance [13] and stochastic synchronization [14]. The role of common noise in enhancing order, with or without periodic forcing, which gives rise to coherence resonance [15], 
is theoretically verified in many systems, such as in climate [16], laser [17], and neuronal $[18,19]$ models. Moreover, the role of noise has been experimentally investigated in excitable and oscillatory coupled BZ systems. Noise-enhanced order and supported wave propagation has been studied in subexcitable photosensitive BZ extended media, where stochastic resonance was observed [20-22]. Noise forcing was studied in BZ reactions by Ohtaki et al. [15], which showed noise-enhanced synchronization of diffuse, weakly coupled, excitable oscillators and in a study by Fukuda et al. [23] that showed optimization of the synchronization order among diffusively coupled BZ oscillators. Noise-enhanced synchronization of coupled oscillators has been experimentally studied in other fields. Examples include coupled chaotic electrochemical oscillators [24, 25], and coupled chaotic laser systems [26].

Noise-induced synchronization was theoretically studied in uncoupled limit-cycle oscillators with white $[27,28]$ and colored noise $[18,29]$, as well as in globally [24, 25] and locally coupled oscillators [30-32], and synchrony of separated populations by climate fluctuations [33]. The synchronized behavior of oscillators driven by common noise was attributed to inducing negative Lyapunov exponents [27, 28, 34], which leads to convergence of nearby phases [35]. A noise-enhanced synchronization demonstrated in a study by Mori and Kai [36] showed that noise enhances brainwave entrainment, which improves visual processing. Moreover, noise-induced synchronization of oscillators has been experimentally studied in uncoupled neurons. A synchronized population of neurons was observed in vitro, where uncoupled mitral cells of mouse olfactory bulbs, which were subjected to aperiodic noise input, became synchronized [37]. In addition, synchronization is observed in vivo with uncoupled sensory neuronal oscillators of paddlefish subjected to a common noise [38]. However, there are 
no experimental studies on noise-induced synchronization of uncoupled oscillatory systems driven purely by a common noise in the BZ reaction or other inanimate systems.

From evidence that points to the possibility of noise-induced synchronization, we chose to use noisy inputs for synchronization of uncoupled BZ oscillators to mimic synaptic background noise that affects neural activity. Our main aim was to allow the synchronized signal to desynchronize in the presence of noise with periodic pulses. Desynchronization of a population of coupled oscillators with double pulses in the presence of noise has been theoretically demonstrated [39]. Our study was motivated by the theoretical neuronal model of Wilson et al. [40], which showed the effectiveness of periodic pulse signals for desynchronization of uncoupled neurons in the presence of noise.

In this Chapter, we forced uncoupled BZ oscillators with a noise signal to obtain synchronized oscillators while stimulating the system with periodic perturbations to cause desynchronization. The forcing we used was colored noise to establish a synchronized signal. We then forced the system with periodic pulses in the presence of the noise signal to test for desynchronization in an open-loop mode that is characteristic of the commonly used DBS [41-43]. We then compared the open-loop method results with the adaptive control method results seen previously in Chapter 3 .

\subsection{Experimental Design}

Three sets of experimental designs were performed with $27 \pm 3$ photosensitive BZ oscillators. The three experimental conditions were noise-forcing, noise with pulse forcing, and no external forcing. The photosensitive catalyst particles were spaced to ensure the oscillators were uncoupled in a catalyst-free BZ solution. Experiments were monitored with a computer 
governing a camera imaging system, with the same setup and preparations described in Section 2.2.1 in Chapter 2.

\subsubsection{Noise-Induced Synchronization Experiments}

Noise signals were used to induce synchronization among uncoupled heterogeneous BZ oscillators. The uncoupled $\mathrm{BZ}$ oscillator reactor was illuminated from a projector with random light intensity values that were adjusted to include lower-frequency colored noise signals. The frequencies were from the visible region of the electromagnetic spectrum. Colored noise can be generated using the Ornstein-Ohlenbeck equation as follows [44]:

$$
\dot{\xi}(t)=-\frac{1}{\tau} \xi+\frac{\sqrt{D}}{\tau} \xi_{w}(t),
$$

where $\xi$ is the colored noise, $\xi_{w}$ is the white noise, $\tau$ is the correlation time scale, and $D$ is noise strength. Figure 4.1a shows an uncorrelated white noise signal produced by a random number generator that yields all frequencies. In the time domain, the amplitudes of random light intensities comprising white noise signals were uniformly distributed around the baseline light intensity. Figure $4.1 \mathrm{~b}$ shows the colored noise signal with frequency components closer to the time scale of the BZ system. Fourier transform power spectra were used to determine the frequency content of the colored noise signal, as shown in Figure 4.2. 

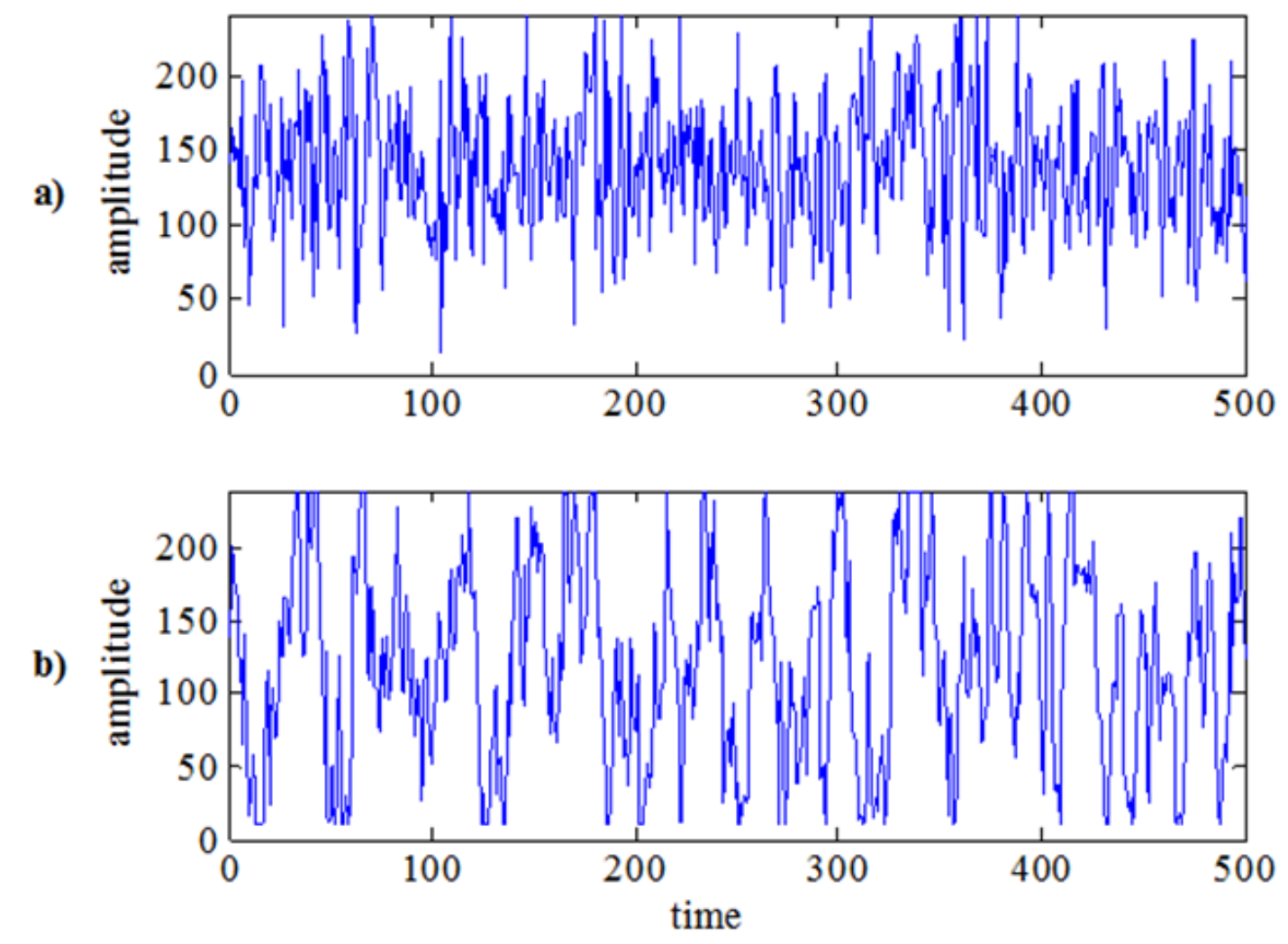

FIG. 4.1: Two typical noise signals. a) White noise with a fast signal comprising all frequencies. b) Colored noise with a slow signal having dominant frequencies.

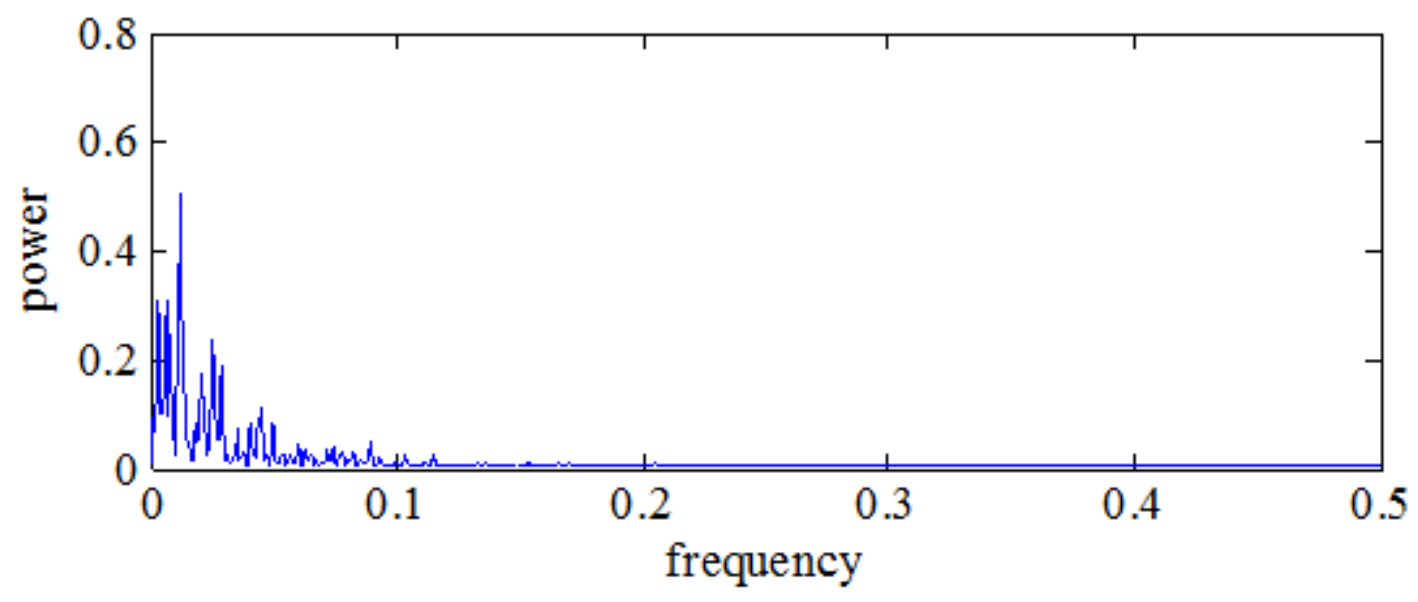

FIG. 4.2: A Fourier transform power spectrum shows lower frequency domains of the colored noise signal. 
In the noise set experiments, the oscillators initially oscillate at their natural frequencies at the background intensity, $I_{0}=1.29 \mathrm{~mW} \mathrm{~cm}^{-2}$, which corresponded to 140 in the gray scale. The illumination of the reactor with the colored noise signal was then introduced at $\mathrm{t}=126.0 \mathrm{~s}$. The intensity of each oscillator was monitored and the values for all oscillators were summed to create a mean signal during the experiment. For comparison purposes, another set of experiments was carried out with the BZ oscillators exposed to a constant background light intensity, $I_{0}$, without a forcing signal.

\subsubsection{Desynchronization Experiments}

In this set of experiments, the photosensitive BZ oscillators with random initial conditions were exposed to both negative pulses and noise signals at $t=126.0 \mathrm{~s}$ as shown in Figure 4.3. The presence of the noise pushes the system toward synchronization, while the periodic negative pulse perturbations give rise to desynchronization under the continuous effect of the noise signal. The negative pulses were of a $9.0 \mathrm{~s}$ offset period adapted to the mean period of control beads oscillating at their natural frequency under constant illumination. The perturbations were applied without knowledge or control of the phase of the synchronized oscillators. The negative intensity pulses had an amplitude of $I=1.29 \mathrm{~mW} \mathrm{~cm}^{-2}$ below the background intensity, which corresponded to a maximum value at the background intensity of 140 to a minimum of 0 in gray scale. The pulses lasted for a quarter of the mean natural period. 


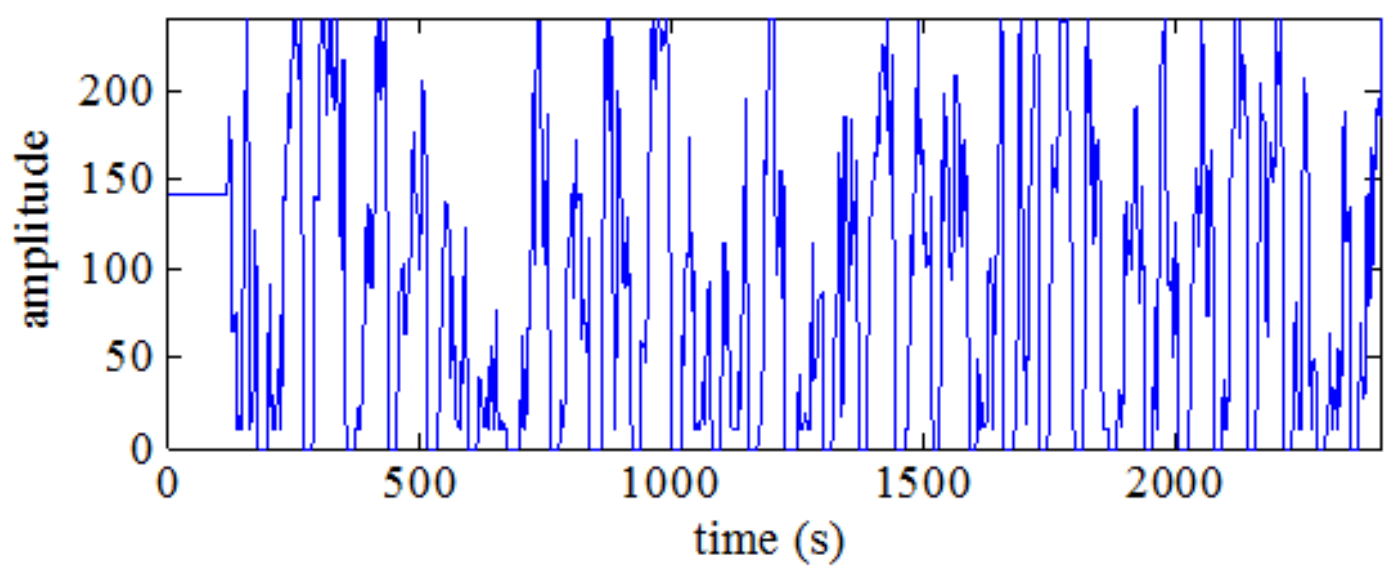

FIG. 4.3: Periodic negative pulses with amplitude $I=1.29 \mathrm{~mW} \mathrm{~cm}^{-2}$ below the background intensity introduced at $\mathrm{t}=126.0 \mathrm{~s}$ along with the colored noise forcing.

To describe the average behavior of purely noise-induced synchronization versus stochastic desynchronization experiments, the three different sets of experiments are compared. Mean order parameters for ten noise-driven experiments, pulse- and noise-driven experiments, and random intrinsic oscillation experiments are reported. The mean and standard deviation of the mean order parameter values of ten representative experiments of each set were calculated. Then, the Student's t-test, which is often used for small data sets, was measured to distinguish differences among two sets of experimental values based on mean, standard deviation, and number of data points. The Formula of the t-test is as follows [45]:

$$
t=\frac{\bar{X}_{1}-\bar{X}_{2}}{\left[\left(\frac{\sigma_{1}^{2}\left(n_{1}-1\right)+\sigma_{2}^{2}\left(n_{2}-1\right)}{n_{1}+n_{2}-2}\right) \times\left(1 / n_{1}+1 / n_{2}\right)\right]^{1 / 2}},
$$

where $\bar{X}_{1}$ and $\bar{X}_{2}$ are the means of the first and second set of experimental values, respectively, with the total number of values $n_{1}$ and $n_{2}$, and $\sigma_{1}$ and $\sigma_{2}$ are standard deviations of the first and second set, respectively. 


\subsection{Experimental Results}

\subsubsection{Signal in the Presence of Noise}

Applying colored noise illumination to uncoupled BZ oscillators provides evidence of a noise-induced synchronization phenomenon. Data were analyzed from the mean signal, individual time series, and order parameter. Figure 4.4a shows regions of enhancement of the mean signal (black plot) when applying colored noise (blue plot). Figure 4.4b shows representative individual time series signals from ten individual oscillators, which reveals coherence in some regions. Figure $4.4 \mathrm{c}$ shows the order parameter with a mean of $r_{\text {mean }}=0.64$, indicating a moderate degree of synchronization.

Our result was consistent with an experimental in vivo study of uncoupled sensory neuronal oscillators, which shows evidence of synchronization by a common noise [38]. In addition, the results are in agreement with predicted behavior from earlier theoretical studies by Pikovsky and coworkers [46, 47], which demonstrate noise-induced synchronization in models of uncoupled identical oscillators driven by random pulse signals and white noise. These studies indicate negative Lyapunov exponents for noise-induced synchronization mechanisms. Our system, however, used uncoupled heterogeneous BZ oscillators driven by colored noise. Noiseinduced coherence was demonstrated with heterogeneity in the theoretical work of Zhou et al. [48]. 
a)

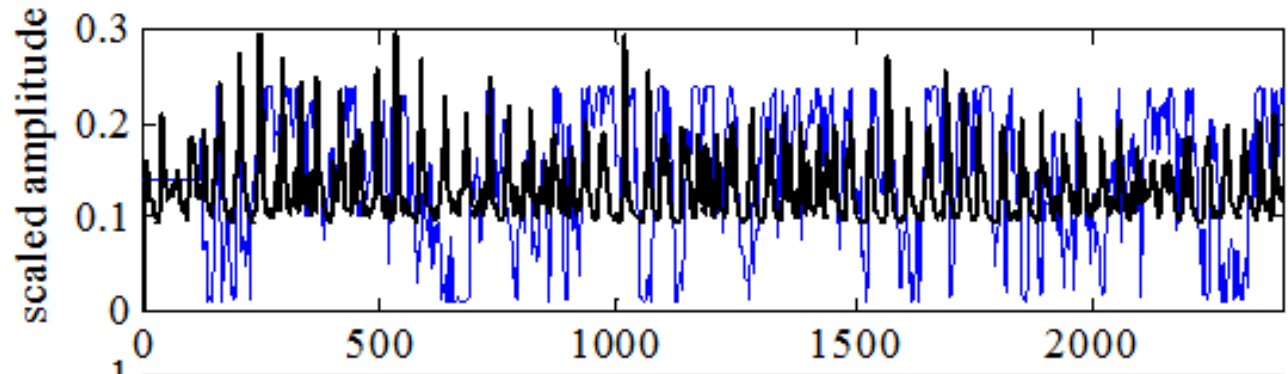

b)

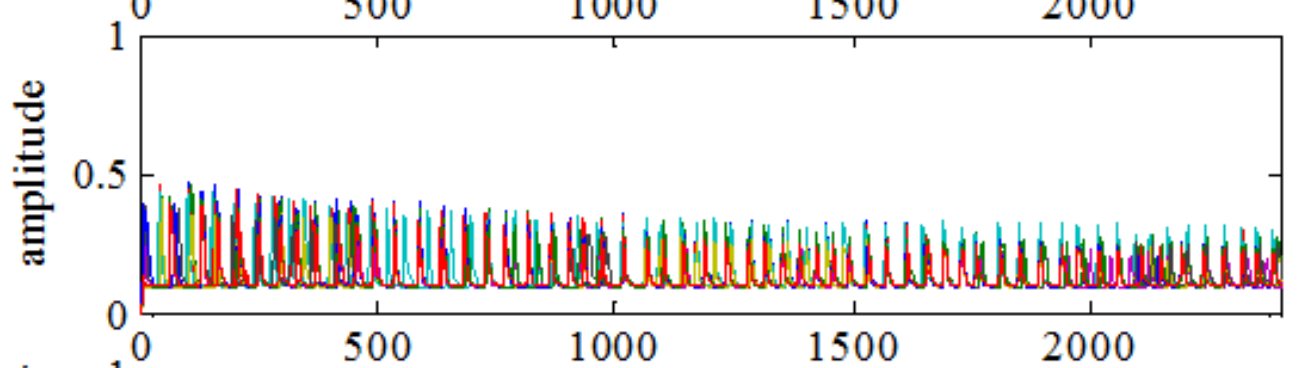

c)

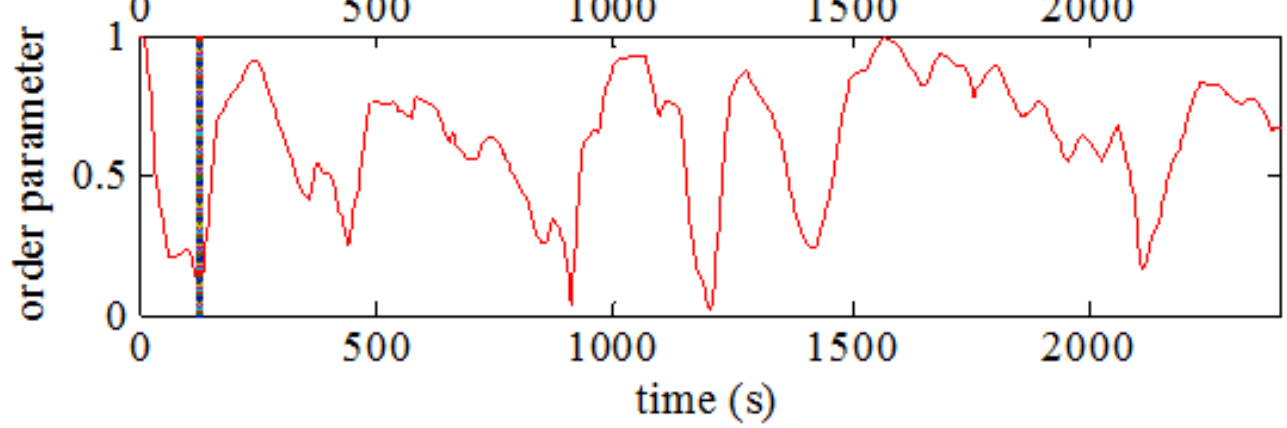

FIG. 4.4: Oscillators synchronized by external colored noise. a) The colored noise light signal (blue) is introduced at $\mathrm{t}=126.0 \mathrm{~s}$. The mean signal (black) shows regions of enhancement, indicating a noiseinduced synchronization phenomenon. b) Individual time series of ten representative oscillators, showing moderate coherence in phase. c) The mean of the order parameter (red) is 0.64 , indicating a moderate degree of synchronization. A system of 30 micro-oscillators loaded with $\mathrm{Ru}(\mathrm{bpy})_{3}^{2+}$ catalyst, with a mean natural period of $63.7 \mathrm{~s}$. The background intensity is $I_{0}=1.29 \mathrm{~mW} \mathrm{~cm}^{-2}$. The BZ reaction mixture composition: malonic acid, $[\mathrm{MA}]=0.08 \mathrm{M}$; sodium bromide, $[\mathrm{NaBr}]=0.02 \mathrm{M}$; sulfuric acid, $\left[\mathrm{H}_{2} \mathrm{SO}_{4}\right]=$ $0.78 \mathrm{M}$; sodium bromate, $\left[\mathrm{NaBrO}_{3}\right]=0.48 \mathrm{M}$.

The behavior observed in a noise-driven system was compared with experiments using BZ oscillators in the absence of noise. Figure 4.5 shows the behavior of uncoupled oscillators with no external forcing. The mean signal is noisy and almost flat, which indicates no synchronized 
behavior. The individual time series shows no coherence in phases, and the order parameter is very low. In addition, applying a Fourier transform to the corresponding mean signals of the noise-forced system and the non-forced system reveals dominate frequencies corresponding to periods of around $50.0 \mathrm{~s}$ with the noise forcing experiment but a lack of a dominate frequency with the non-forced system, as shown in Figure 4.6.

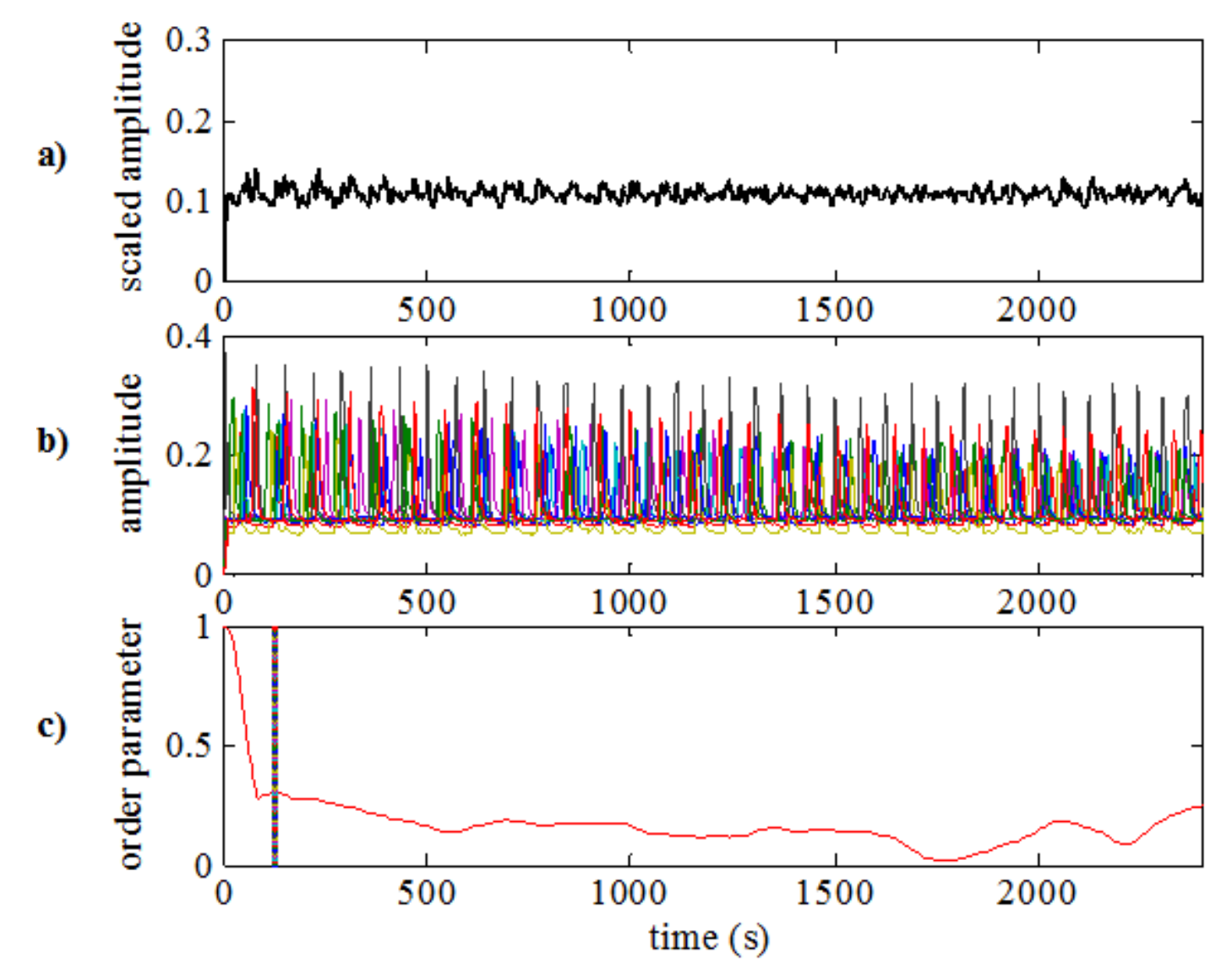

FIG. 4.5: Intrinsic oscillations of uncoupled BZ oscillators. a) The oscillators are maintained at a constant background light intensity. The mean signal (black) shows a noisy signal, indicating no synchronization. b) Individual time series of ten representative oscillators show non-coherent phases. c) The mean of the order parameter (red) is 0.14 , indicating no synchronization. System of 30 micro-oscillators loaded with $\mathrm{Ru}(\mathrm{bpy})_{3}^{2+}$ catalyst, with a mean natural period of $85.8 \mathrm{~s}$. The background intensity is $I_{0}=1.29 \mathrm{~mW} \mathrm{~cm}^{-2}$. The BZ reaction mixture composition is the same as in Figure 4.4. 

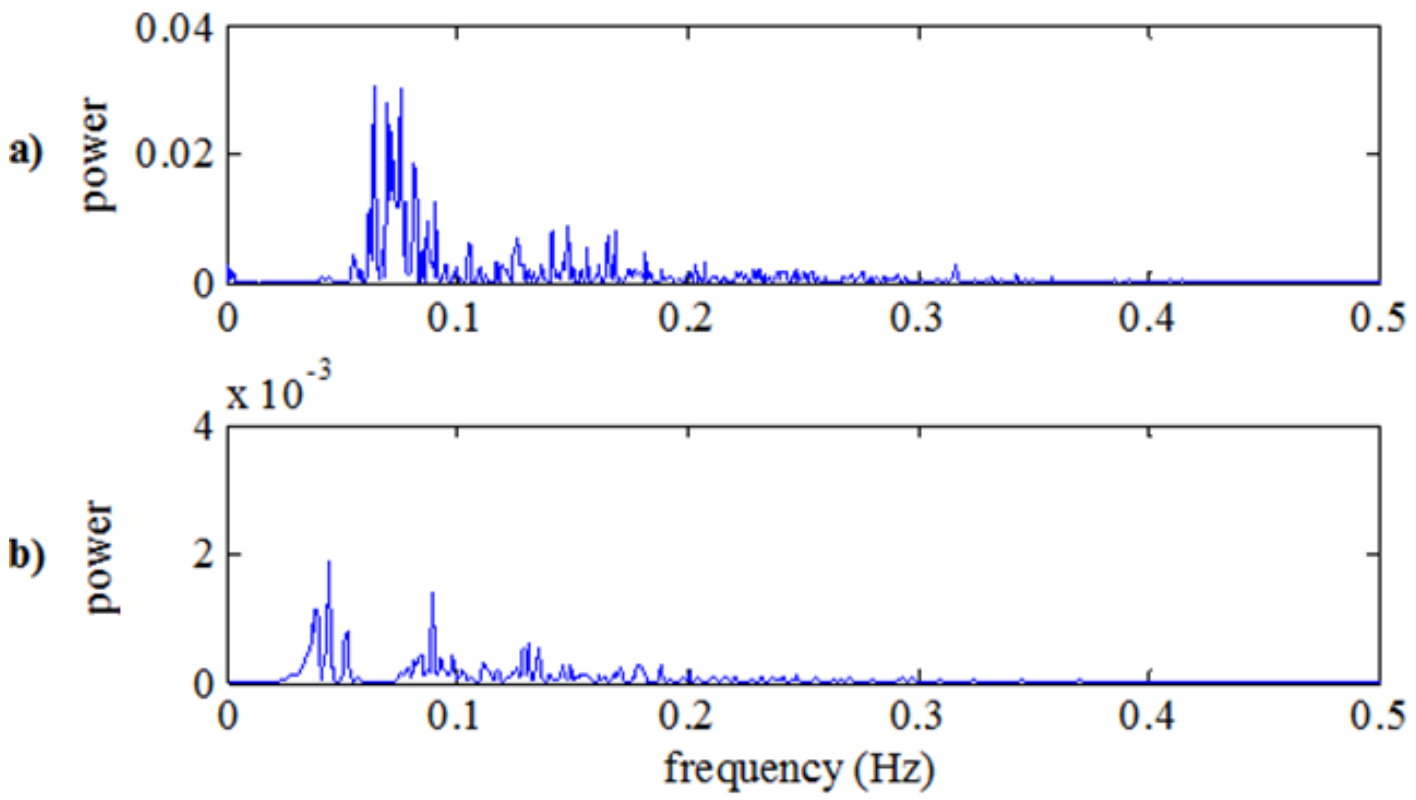

FIG. 4.6: Fourier transform power spectra for noise-driven and autonomous BZ systems. a) The power spectrum of the mean signal from a noise-driven BZ system shows a narrow range of dominate frequencies, indicating noise-induced synchronization. b) The power spectrum of the mean signal from intrinsic oscillations of uncoupled BZ oscillators shows the lack of a dominant frequency, indicating no synchronization when no forcing signal is applied.

\subsubsection{Signal in the Presence of Noise and Negative Perturbations}

Applying negative pulses in the presence of a noise signal leads to desynchronization. Figure 4.7 shows the mean signal is an irregular noisy signal when negative pulses are applied, indicating desynchronization. The time series of ten representative oscillators show non-coherent phases and a mean order parameter of 0.24 , which indicates desynchronization. 
a)

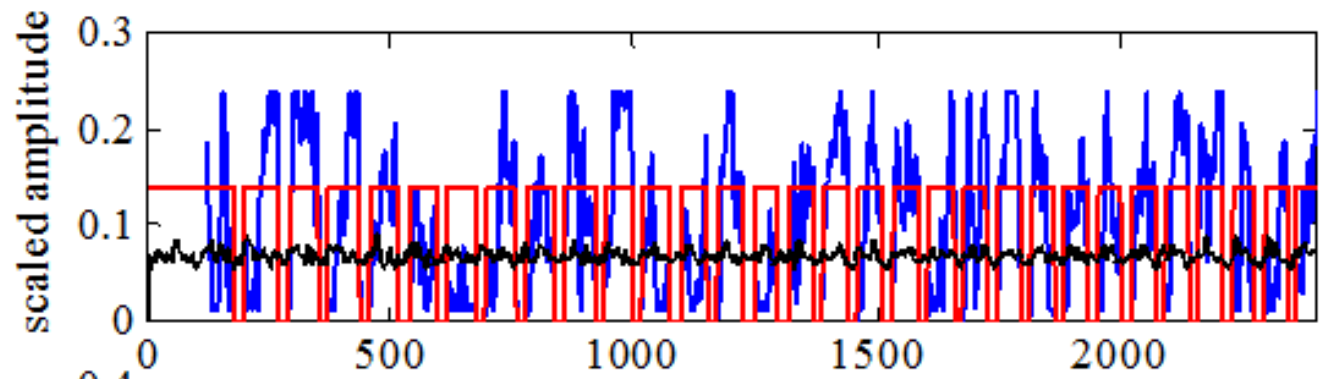

b)

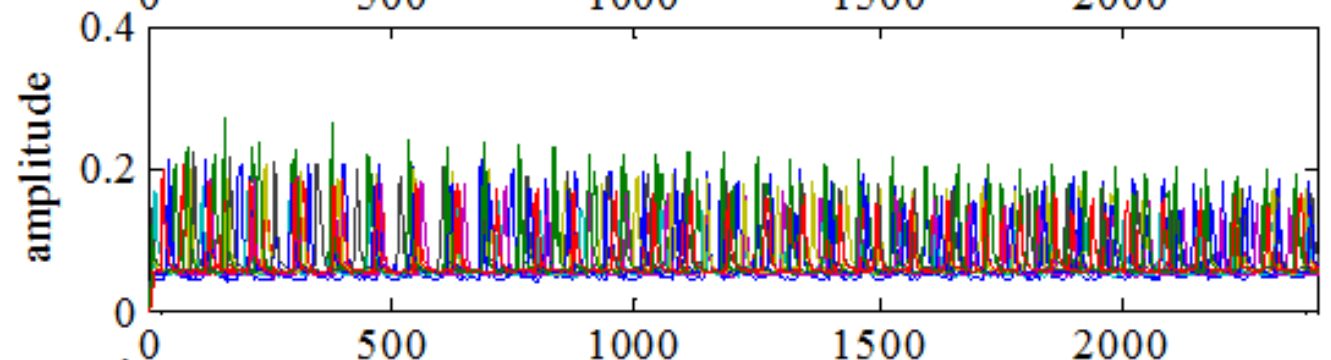

c)

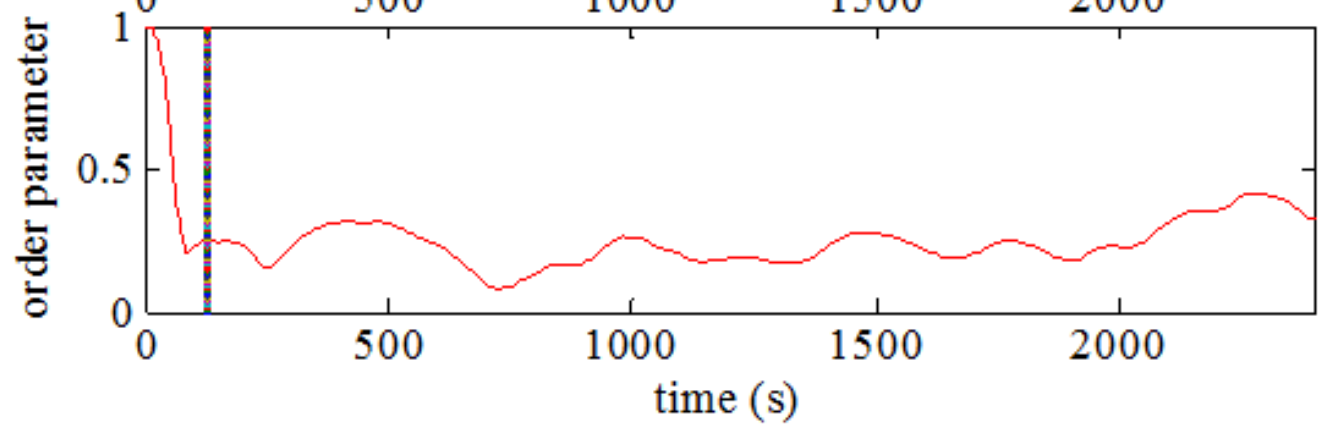

FIG. 4.7: Uncoupled BZ oscillators subjected to both negative pulses and colored noise. a) Negative pulses (red) and noise signals introduced at $t=126.0 \mathrm{~s}$ (blue). The mean signal (black) shows a noisy signal, indicating desynchronization. b) The time series of ten representative oscillators show noncoherent phases. c) The mean order parameter (red) is 0.24 , which is low and indicates desynchronization. System of 30 micro-oscillators loaded with $\mathrm{Ru}(\mathrm{bpy})_{3}^{2+}$ catalyst, with a mean natural period of $86.0 \mathrm{~s}$. The background intensity is $I_{0}=1.29 \mathrm{~mW} \mathrm{~cm}^{-2}$. The $\mathrm{BZ}$ reaction mixture composition is the same as in Figure 4.4.

We compared the results of the three different experiments by performing ten experiments in each set and calculated the mean order parameters, as shown in Table 4.1. Group A represents experiments with noise forcing, where the average mean order parameter values of ten experiments is 0.53 , indicating significant synchronization. Group B represents experiments with 
noise and negative pulse forcing, where the average mean order parameter values of ten experiments is 0.27 , indicating desynchronization. Group $\mathrm{C}$ represents experiments with no forcing, where the average mean order parameter values of ten experiments is 0.16 , indicating no synchronization.

Table 4.1: Data of Mean Order Parameters Values in Each Set of Experiments.

\begin{tabular}{|c|c|c|c|c|c|c|}
\cline { 2 - 7 } \multicolumn{1}{c|}{} & \multicolumn{2}{c|}{ A } & \multicolumn{2}{c|}{ B } & \multicolumn{2}{c|}{ C } \\
\hline Experiments Forcing & $\begin{array}{c}\text { Mean order } \\
\text { parameter }\end{array}$ & $\begin{array}{c}\text { Number } \\
\text { of beads }\end{array}$ & $\begin{array}{c}\text { Mean order } \\
\text { parameter }\end{array}$ & $\begin{array}{c}\text { Number } \\
\text { of beads }\end{array}$ & $\begin{array}{c}\text { Mean order } \\
\text { parameter }\end{array}$ & $\begin{array}{c}\text { Number } \\
\text { of beads }\end{array}$ \\
\hline 1 & 0.44 & 30 & 0.26 & 30 & 0.13 & 28 \\
2 & 0.57 & 30 & 0.28 & 30 & 0.17 & 27 \\
3 & 0.64 & 30 & 0.24 & 30 & 0.13 & 30 \\
4 & 0.47 & 30 & 0.20 & 30 & 0.15 & 30 \\
5 & 0.43 & 30 & 0.20 & 30 & 0.18 & 30 \\
6 & 0.66 & 25 & 0.22 & 30 & 0.14 & 30 \\
7 & 0.58 & 30 & 0.27 & 30 & 0.16 & 25 \\
8 & 0.48 & 25 & 0.30 & 30 & 0.20 & 26 \\
\hline 9 & 0.55 & 25 & 0.33 & 30 & 0.21 & 30 \\
\hline 10 & 0.49 & 30 & 0.36 & 30 & 0.16 & 25 \\
\hline Average & \multicolumn{2}{|c|}{0.53} & \multicolumn{2}{c|}{0.27} & & 0.16 \\
\hline SD & 0.08 & \multicolumn{2}{c|}{0.05} & & 0.03 \\
\hline
\end{tabular}

We further performed a statistical analysis of the average behavior of both sets using ttests, as shown in Table 4.2. The tests showed that there is a significant difference among two sets of the three different experiments with regard to the mean order parameter values. The calculated values of the t-test in all two-grouped sets exceeded the corresponding tabulated value of 3.92 for probability of $\mathrm{p}=0.0005$ [49], suggesting a $99.95 \%$ chance that the average of the mean order parameters of different sets were significantly different from each other. Noisedriven experiments were shown to have different results than experiments with noise and pulse- 
driving signals and those with no driving signal, which indicates the ability of a purely common noise signal to induce synchronization among uncoupled BZ oscillators. It also shows the ability of negative pulses for desynchronization in an open-loop fashion.

Table 4.2: Calculated t-test from Two Different Sets of Experiments $(\mathrm{A}=$ noise-forcing experiments; $\mathrm{B}=$ negative pulse and noise-forcing experiments; $\mathrm{C}=$ no forcing experiments).

\begin{tabular}{|c|c|}
\hline Experiments Sets & Student' t-test \\
\hline A \& B & 8.7 \\
\hline A \& C & 13.6 \\
\hline B \& C & 5.48 \\
\hline
\end{tabular}

Moreover, we tested the effects of applying positive periodic pulse perturbations in the presence of noise. The results show that applying positive pulses in the presence of noise does not lead to desynchronization. Figure 4.8a shows an enhancement of the mean signal when applying both positive pulses and colored noise. Figure $4.8 \mathrm{~b}$ shows representative individual time series signals from ten individual oscillators, which reveals phase coherence. Figure $4.8 \mathrm{c}$ shows the order parameter with a mean of 0.85 , indicating a high degree of synchronization. 
a)

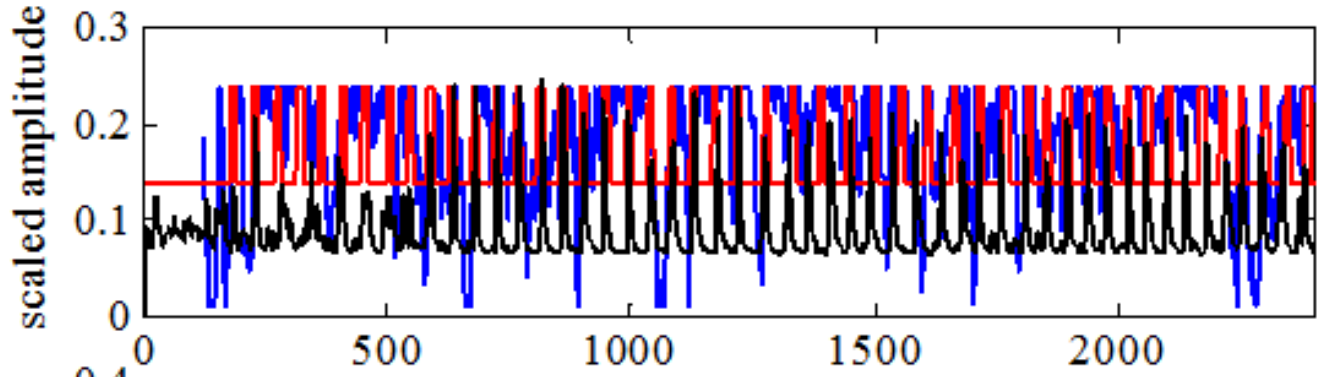

b)

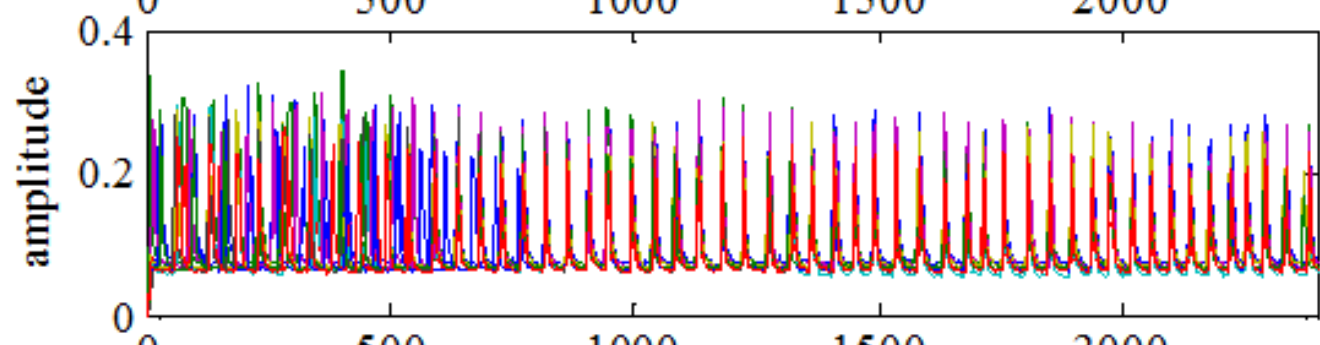

c)

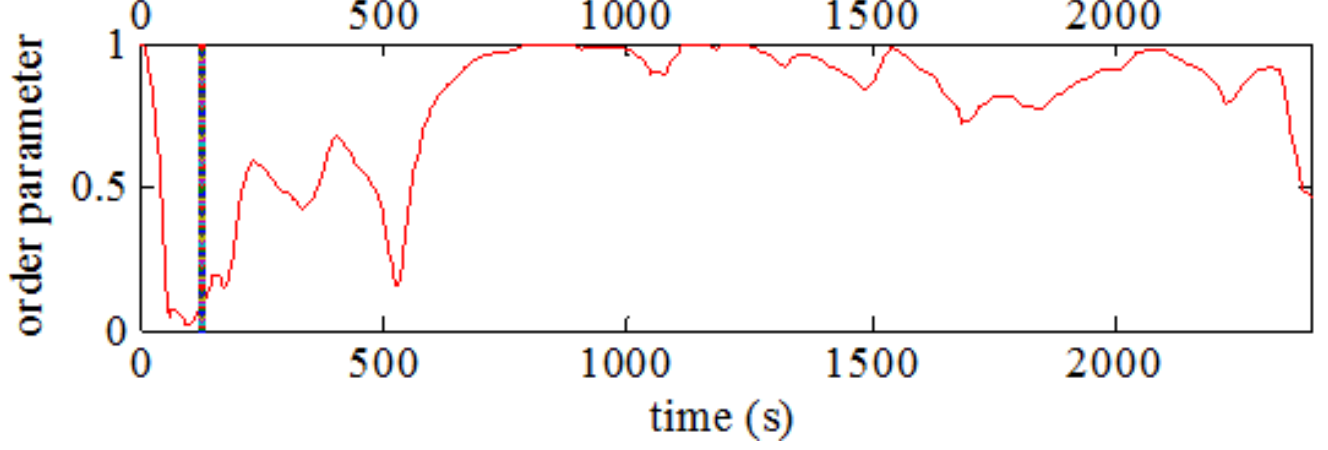

FIG. 4.8: Oscillators synchronized by external positive pulses in the presence of noise. a) Positive pulses (red) and colored noise light signal (blue) introduced at $t=126.0 \mathrm{~s}$. The mean signal (black) shows enhancements and regular signal, indicating synchronization. b) The time series of ten representative oscillators show aligned coherence in phase. c) The mean of the order parameter (red) is 0.85 , indicating a high degree of synchronization. System of 27 micro-oscillators loaded with $\mathrm{Ru}(\mathrm{bpy})_{3}^{2+}$ catalyst, with mean natural period of $69.4 \mathrm{~s}$. The background intensity is $I_{0}=1.29 \mathrm{~mW} \mathrm{~cm}^{-2}$. The BZ reaction mixture composition is the same as in Figure 4.4.

The observed behavior is analogous to the phenomenon of "stochastic synchronization," where noise- and periodic-forcing signals are applied together to enhance weak signals. Stochastic synchronization was theoretically demonstrated in a model of an uncoupled oscillator array, where the mean frequency from the mean signal was locked to the forcing signal due to 
the presence of noise [50]. In addition, the behavior was shown numerically in coupled network systems, where stochastic synchronization among networks occurred in the presence of noise [51]. In contrast to previous theoretical work, our results show that synchronization of a discrete uncoupled system forced by a positive square wave signal could be maintained in the presence of noise.

\subsection{Discussion}

Random signals in the noisy environment of neurons have been experimentally demonstrated to mediate synchronization $[2,4]$. We have investigated the effect of colored noise in inducing synchronization. We used uncoupled BZ oscillators subjected to a common noise signal and then stimulated this system via periodic negative pulses to investigate a desynchronization mechanism for DBS $[39,40]$. Our results demonstrated the ability of a noise signal to induce synchronization. This resembles the theoretical study by Teramae and Tanaka [27], which showed the ability of noise to induce order in independent limit cycle oscillators. Our results correlate with results from in vitro and in vivo experiments, respectively, where noise was shown to induce order [37, 38]. Moreover, the behavior is in agreement with other experimental studies. For instance, an experimental study by Zhou et al. [24], which investigated the effect of noise with no other external forcing, showed that synchronization is obtained in coupled chaotic oscillators in the presence of noise. Noise has been shown to facilitate wave propagation in BZ extended excitable media [20]. Noise-enhanced synchronization was shown in a BZ reaction [15] of weakly and diffusively coupled excitable oscillators, and in the Fukuda et al. study [23] that showed optimization in the order of synchronization among weakly and diffusively coupled BZ oscillators. However, there are no experimental studies on the 
synchronization of discrete and uncoupled BZ oscillators or other uncoupled non-biological oscillators driven by common noise.

The application of a noisy signal is different than applying a regular pulse or a sine-wave signal in this study, because it does not depend on the continuous measurement of oscillator frequency. When comparing this system to our previous periodic stimulus study in Chapter 3 , we find that the heterogeneous system of uncoupled BZ oscillators is less sensitive to the common noise input than a common regular positive signal. Synchronization to a noisy input occurs, but synchronization is not complete. The mean of the order parameters of ten experiments was moderate and significantly different from the mean of the order parameters of non-forced systems and systems that underwent regular negative forcing. Moreover, we find that the introduction of a negative stimulus promotes desynchronization. The degree of desynchronization is distinct, but less than complete desynchronization where no stimulus is applied. Noise with pulse-driving experiments were carried out in an open-loop fashion as opposed to the earlier pulse-driving experiments, where the oscillators' phases were synchronized with a positive stimulus prior to administration of a desynchronizing stimulus.

The application of periodic positive pulses in the presence of noise was shown to lead to a different observation than when negative pulses were applied in the presence of noise. The behavior can be discussed in terms of the Lyapunov exponents, where negative Lyapunov exponents lead to convergence of nearby oscillators and eventual synchronization, while positive Lyapunov exponents cause nearby oscillators to diverge, thereby leading to desynchronization [35]. The positive pulses lead only to a negative slope in the phase response curve (PRC), while the PRC with negative pulses has both negative and positive slopes. Although negative perturbations are periodic and applied to random phases, desynchronization is observed in the 
noise forcing system. This can be understood based on the PRC slope caused by negative perturbations, where more of the curve has a positive slope. The positive slope occupied two thirds of the PRC, while the negative slope, responsible for synchronization, occupied only a third of the PRC, as shown in Figure 3.1 in Chapter 3. Therefore, our system may show desynchronization with negative pulses, because the probability of phase divergence becomes dominant over phase convergence.

Although the inclusion of both periodic and noisy components in much of the literature suggests a stochastic resonance phenomenon $[2,18,20,21,50]$ or stochastic synchronization $[4$, $36,51]$, our results indicate that the application of negative pulses in the presence of noise leads to stochastic desynchronization.

\subsection{Summary}

In this chapter, we have demonstrated the synchronizing of uncoupled BZ oscillators by a common colored noise signal. An open-loop desynchronization stimulation of BZ synchronized oscillators was considered. We showed that negative perturbations cause desynchronization even when applying the perturbations in an open-loop manner. This is due to regions in the PRC giving rise to divergence by negative perturbations.

The synchronization/desynchronization results are displayed via mean signals, individual time series, order parameters, and t-test calculations. These results support the theoretical desynchronization mechanism of DBS over the entrainment mechanism, as in the theoretical neuronal model by Wilson et al. [40], which suggested the effectiveness of periodic pulse signals for desynchronization of uncoupled neurons in the presence of noise. However, our results suggest that the pulse type is a significant factor for effective desynchronization via establishing positive Lyapunov exponents. The periodic negative pulse desynchronization can be introduced 
to desynchronize the abnormal synchronous oscillations in neuron circuits responsible for tremors in Parkinson's disease [9-11]. Future studies can facilitate closed-loop desynchronization methods in the presence of noise. Positive pulse perturbations can be administrated first to bring the system to a well-defined synchronized signal. An experimental design can be created to allow for the control of the perturbations based on read-time monitoring of the maxima of the mean signal in the presence of random fluctuations.

In conclusion, our main results are that appropriate common noise induces synchronization, while appropriate common regular signals can induce desynchronization among independent BZ oscillators. 


\section{Reference}

[1] L. Glass, "Synchronization and rhythmic processes in physiology." Nature 410, 277$284(2001)$

Online Version

[2] Z. F. Mainen and T. J. Sejnowski, "Reliability of spike timing in neocortical neurons." Science 268, 1503-1506 (1995).

Online Version

[3] A. Destexhe, M. Rudolph, J-M. Fellous, and T. J. Sejnowski, "Fluctuating synaptic conductances recreate in vivo-like activity in neocortical neurons." Neuroscience 107, 13-24 (2001).

Online Version

[4] J-M. Fellous, M. Rudolph, A. Destexhe, and T. J. Sejnowski, "Synaptic background noise controls the input/output characteristics of single cells in an in vitro model of in vivo activity." Neuroscience 122, 811-829 (2003).

Online Version

[5] S. H. Strogatz, "The mathematical structure of the human sleep-wake cycle." In S. Levin (Ed.), Lecture Notes in Biomathematics 69 (Springer-Verlag, Berlin, 1986). Online Version

[6] R. Wilders and H. J. Jongsma, "Beating irregularity of single pacemaker cells isolated from the rabbit sinoatrial node.” Biophys. J. 60, 2601-2613 (1993).

Online Version

[7] G. A. Petrillo and L. Glass, "A theory for phase-locking of respiration in cats to a mechanical ventilator.” Am. J. Physiol. 246, R311-R320 (1984). 
Online Version

[8] C. Hammond, H. Bergman, and P. Brown, "Pathological synchronization in Parkinson's disease: Networks, models and treatments." Trends Neurosci. 30, 357-364 (2007).

Online Version

[9] J. Volkmann, M. Joliot, A. Mogilner, A. Ioannides, F. Lado, E. Fazzini, U. Ribary, and R. Llinás, "Central motor loop oscillations in Parkinsonian resting tremor revealed by magnetoencephalography." Neurology 46, 1359-1370 (1996).

Online Version

[10] H. Bergman, A. Raz, A. Feingold, A. Nini, I. Nelken, B-P. Hilla, D. Hansel, and A. Reches, "Physiology of MPTP tremor.” Mov. Disord. 13, 29-34 (1998).

Online Version

[11] R. Levy, W. Hutchison, A. Lozano, and J. Dostrovsky, "High-Frequency synchronization of neuronal activity in the subthalamic nucleus of Parkinsonian patients with limb tremor.” J. Neurosci. 20, 7766-7775 (2000).

Online Version

[12] A. Nini, A. Feingold, H. Slovin, and H. Bergman, "Neurons in the globus pallidus do not show correlated activity in the normal monkey, but phase-locked oscillations appear in the MPTP model of Parkinsonism.” J. Neurophysiol. 74, 1800-1805 (1995).

Online Version

[13] K. Wiesenfeld and F. Moss, "Stochastic resonance and the benefits of noise: From ice ages to crayfish and SQUIDs.” Nature 373, 33-36 (1995).

Online Version 
[14] A. S. Pikovsky, "Synchronization and stochastization of array of self-excited oscillators by external noise.” Radiophys. Quantum El. 27, 390-395 (1984).

Online Version

[15] M. Ohtaki, T. Tanaka, and K. Miyakawa, 'Noise-induced phase locking in coupled coherence-resonance oscillators.” Phys. Rev. E 70, 056219 (2004).

Online Version

[16] A. Ganopolski and S. Rahmstorf, "Abrupt glacial climate changes due to stochastic resonance.” Phys. Rev. Lett. 88, 038501 (2002).

Online Version

[17] J. M. Buldú, J. García-Ojalvo, C. R. Mirasso, M. C. Torrent, and J. M. Sancho, "Effect of external noise correlation in optical coherence resonance.” Phys. Rev. E 64, 051109 (2001).

Online Version

[18] J. D. Hunter, J. G. Milton, P. J. Thomas, and J. D. Cowan, "Resonance effect for neural spike time reliability.” J. Neurophysiol. 80, 1427-1438 (1998).

Online Version

[19] E. Schneidman, B. Freedman, and I. Segev, "Ion channel stochasticity may be critical in determining the reliability and precision of spike timing." Neural Comput. 10, 16791703 (1998).

Online Version

[20] S. Kádár, J. Wang, and K. Showalter, "Noise-supported travelling waves in subexcitable media." Nature 391, 770-772 (1998).

Online Version 
[21] P. Jung, A. Cornell-Bell, F. Moss, S. Kádár, J. Wang, and K. Showalter, "Noise sustained waves in subexcitable media: From chemical waves to brain waves." Chaos 8, 567-575 (1998).

Online Version

[22] J. Wang, S. Kádár, P. Jung, and K. Showalter, "Noise driven avalanche behavior in subexcitable media." Phys. Rev. Lett. 82, 855-858 (1999).

Online Version

[23] H. Fukuda, H. Morimura, and S. Kai, "Global synchronization in two-dimensional lattices of discrete Belousov-Zhabotinsky oscillators." Phys. D 205, 80-86 (2005). Online Version

[24] C. S. Zhou, J. Kurths, I. Kiss, and J. Hudson, "Noise-enhanced phase synchronization of chaotic oscillators." Phys. Rev. Lett. 89, 014101 (2002).

Online Version

[25] I. Kiss, Y. Zhai, J. Hudson, C. Zhou, and J. Kurths, "Noise enhanced phase synchronization and coherence resonance in sets of chaotic oscillators with weak global coupling." Chaos 13, 267-278 (2003).

Online Version

[26] C. S. Zhou, J. Kurths, E. Allaria, S. Boccaletti, R. Meucci, and F. T. Arecchi, “Constructive effects of noise in homoclinic chaotic systems.” Phys. Rev. E 67, 066220 (2003).

Online Version

[27] J. Teramae, and D. Tanaka, "Robustness of the noise-induced phase synchronization in a general class of limit cycle oscillators.” Phys. Rev. Lett. 93, 204103 (2004). 
Online Version

[28] J. Ritt, "Evaluation of entrainment of a nonlinear neural oscillator to white noise." Phys. Rev. E 68, 041915 (2003).

Online Version

[29] D. S. Goldobin, J. Teramae, H. Nakao, G. B. Ermentrout, "Dynamics of limit-cycle oscillators subject to general noise.” Phys. Rev. Lett. 105, 154101 (2010).

Online Version

[30] E. Ullner, R. Vicente, G. Pipa, J. Garcia-Ojalvo, "Contour integration and synchronization in neuronal networks of the visual cortex." In Artificial Neural Networks - ICANN 2008, edited by V. Kůrková, R. Neruda, and J. Koutník (Springer, Berlin Heidelberg, 2008), pp. 703-712.

Online Version

[31] A. Neiman, L. Schimansky-Geier, A. Cornell-Bell, and F. Moss, "Noise-enhanced phase synchronization in excitable media.” Phys. Rev. Lett. 83, 4896-4899 (1999). Online Version

[32] J. F. Lindner, B. K. Meadows, W. L. Ditto, M. E. Inchiosa, and A. R. Bulsara, "Scaling laws for spatiotemporal synchronization and array enhanced stochastic resonance." Phys. Rev. E 53, 2081-2086 (1996).

Online Version

[33] B. T. Grenfell, K. Wilson, B. F. Finkenstädt, T. N. Coulson, S. Murray, S. D. Albon, J. M. Pemberton, T. H. Clutton-Brock, and M. J. Crawley, “ Noise and determinism in synchronized sheep dynamics.” Nature 394, 674-677 (1998).

Online Version 
[34] R. V. Jensen, "Synchronization of randomly driven nonlinear oscillators." Phys. Rev. E 58, R6907-R6910 (1998).

Online Version

[35] A. Pikovsky, M. Rosenblum, and J. Kurths, Synchronization: A universal concept in nonlinear sciences (Cambridge University Press, Cambridge, 2003).

Online Version

[36] T. Mori and S. Kai, "Noise-induced entrainment and stochastic resonance in human brain waves.” Phys. Rev. Lett. 88, 218101 (2002).

Online Version

[37] R. F. Galán, N. Fourcaud-Trocmé, G. B. Ermentrout, N. N. Urban, "Correlationinduced synchronization of oscillations in olfactory bulb neurons." J. Neurosci. 26, 3646-3655 (2006).

Online Version

[38] A. Neiman and D. Russell, "Synchronization of noise-induced bursts in noncoupled sensory neurons.” Phys. Rev. Lett. 88, 138103 (2002).

Online Version

[39] P. A. Tass, "Desynchronizing double-pulse phase resetting and application to deep brain stimulation.” Biol. Cybern. 85, 343-354 (2001).

Online Version

[40] C. Wilson, B. Beverlin, and T. Netoff, "Chaotic desynchronization as the therapeutic mechanism of deep brain stimulation.” Front. Syst. Neurosci. 5, 00050 (2011).

Online Version 
[41] P. Limousin, P. Pollak, A. Benazzouz, D. Hoffmann, J. F. Le Bas, E. Broussolle, J. E. Perret, and A. L. Benabid, "Effect of Parkinsonian signs and symptoms of bilateral subthalamic nucleus stimulation.” Lancet 345, 91-95 (1995).

Online Version

[42] R. Kumar, A. M. Lozano, Y. J. Kim, W. D. Hutchison, E. Sime, E. Halket, and A. E. Lang, "Double-blind evaluation of subthalamic nucleus deep brain stimulation in advanced Parkinson's disease.” Neurology 51, 850-855 (1998).

Online Version

[43] A. Benazzouz, C. Gross, J. Féger, T. Boraud, and B. Bioulac, "Reversal of rigidity and improvement in motor performance by subthalamic high-frequency stimulation in MPTP-treated Monkeys.” Eur. J. Neurosci. 5, 382-389 (1993).

Online Version

[44] P. Hänggi, P. Jung, C. Zerbe, and F. Moss, "Can colored noise improve stochastic resonance?” J. Stat. Phys. 70, 25-47 (1993).

Online Version

[45] B. K. Moser and G. R. Stevens, "Homogeneity of variance in the two-sample means test.” Am. Stat. 46, 19-21 (1992).

Online Version

[46] A. S. Pikovsky, M. G. Rosenblum, G. V. Osipov, and J. Kurths, "Phase synchronization of chaotic oscillators by external driving.” Phys. D 104, 219-238 (1997). Online Version

[47] D. S. Goldobin and A. S. Pikovsky, "Synchronization of self-sustained oscillators by common white noise.” Phys. A 351, 126-132 (2005). 
Online Version

[48] C. Zhou, J. Kurths, and B. Hu, "Array-enhanced coherence resonance: Nontrivial effects of heterogeneity and spatial independence of noise." Phys. Rev. Lett. 87, 098101 (2001).

Online Version

[49] Texas A\&M University, Texas, "Tables: APPENDIX Table 2 Percentage points of Student's t distribution.” TEACHING 651 - Texas A\&M Study Resources, http://www.stat.tamu.edu/ suhasini/teaching651/tables.pdf (2002).

[50] A. Neiman, F. Moss, L. Schimansky-Geier, and W. Ebeling, "Synchronization in ensembles of stochastic resonators.” AIP Conf. Proc. 411, 151-156 (1997).

Online Version

[51] R. K. Esfahani, F. Shahbazi, and K. A. Samani, "Noise-induced synchronization in small world networks of phase oscillators.” Phys. Rev. E 86, 036204 (2012).

Online Version 


\section{Chapter 5}

\section{Phase-Lag Synchronization in a Chemical Oscillator Network}

\subsection{Introduction}

Neural clustering mediated by network connections has been suggested to be important for brain functions [1]. This view is supported by a number of studies in visual perception $[2,3]$, sound processing [4], information coding [1], and motor rhythm experiments [5]. Synchronization can also have pathological consequences. For example, cluster synchronization of neurons correlates with tremors in Parkinson's disease [6]. Accordingly, studying cluster synchronization in discrete systems is important from a biological point of view. Clusters and phase-synchronization patterns have been studied in a BZ reaction system via heterogeneous coupling through both inhibitory and excitatory species [7], or heterogeneity of non-identical oscillators [8]. Taylor et al. [7] demonstrated phase-cluster states in the global coupling of stirred non-identical BZ oscillators. Moreover, Taylor et al. [8] observed phase clusters in globally coupled heterogeneous populations of discrete photochemical BZ oscillators. Phase-cluster synchronization has also been studied in other systems. Cluster formation has been observed experimentally in coupled electrochemical oscillators [9-11] and in coupled lasers with unidirectional ring connections when applying time-delayed feedback [12]. Moreover, the underling symmetry of phase-cluster formation was experimentally demonstrated by Pecora $e t$ al. [13] in a network of identical optical oscillators.

In this study, we were motivated by Pecora et al. [13] who showed how symmetry plays a role in cluster synchronization. Our study of a network of non-identical oscillators in a lightsensitive BZ set-up reveals a new type of wave-like phase synchronization. This phase-lag 
synchronization has not been observed before in non-continuous media or in a network. The behavior correlates with the suggested mechanism of motor patterns, where, in addition to zerolag synchronization within segments, phase-lag synchronization [14] between segments becomes a mechanism for coherent motion $[15,16]$. Examples include the swimming of fish via fixedphase relations $[17,18]$ and with non-precise phasing during leech crawling [19-21]. It is also known that phase waves, with constant phase-lag relationships, are a local response of participating motor neurons through network connectivity, which is governed by neural circuits called "central pattern generators" $[16,22]$.

Phase waves were first predicted for oscillatory chemical reactions in 1973 [23]. This was later experimentally demonstrated in 1987 in a BZ continuous system perturbed with laser beams by Bodet et al. [24], where a phase wave occurred as a result of imposed differences in reaction rates. In 1994, Aliev observed phase waves in a light-sensitive BZ reaction [25]. A study of a spatially extended oscillatory BZ system showed regions of shifted phase with light illumination control [26]. A theoretical study of limb movements has shown that bidirectional network connections can lead to constant phase-lag cluster synchronization [27].

In this work, we report on experimentally observed phase-lag cluster synchronization, where groups of phase-coherent clusters were sequentially formed with a constant phase difference. Clusters have phase shifts that, in total, cover only part of the phase cycle of $2 \pi$. A cluster can be composed of one or more oscillators. In phase-lag cluster synchronization, each cluster is made up of phase synchronized oscillators which are frequency synchronized with all other clusters. In this chapter, we discuss different types of synchronization behaviors observed experimentally in a network of heterogeneous photochemical BZ oscillators with bidirectional coupling. 


\subsection{Network Experimental Design}

In this set of experiments, we use a small bidirectional network of 10 nodes, as shown in Figure 5.1. The network has a number of symmetrical permutation properties, where collections of nodes, or a symmetry cluster, can be exchanged without changing the original topology [28]. Large networks with non-trivial symmetries can be obtained by using computer software [29]. The experiment was conducted by placing photosensitive oscillators of broad frequency heterogeneity into a catalyst-free BZ solution. Details of the set-up and experimental preparations were the same as shown in Section 2.2.1 in Chapter 2.

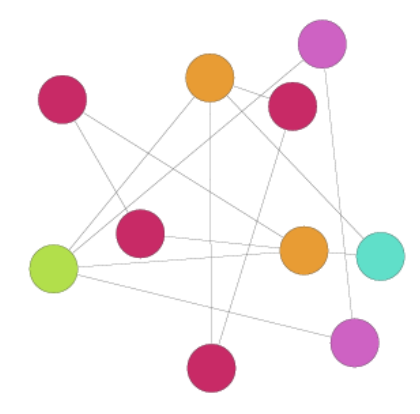

FIG. 5.1: A bidirectional 10-node network. Nodes that are the same color represent a symmetry cluster, and links (gray lines) represent the network connectivity.

Positive photochemical feedback was used to provide coupling via activator species production [8]. Each oscillator was recorded by an imaging system composed of a chargecoupled device camera attached to a spatial light modulator projector, which was controlled by a computer. The transmitted light from an oscillator measured in gray scale was used to determine the feedback. The calculated feedback illumination was projected onto each oscillator.

The following equation was used to calculate the feedback illumination $\varphi_{i}$ for each node $i$ of the $N$ node network: 


$$
\varphi_{i}=\varphi_{0}+\frac{\mathrm{K}}{k_{i}} \sum_{j=1}^{N} A_{i j}\left(z_{j}-z_{i}\right)
$$

The network topology is defined by an adjacency matrix $A_{i j}$. The feedback is determined from the differences in light intensities of the coupled oscillators $\left(z_{j}-z_{i}\right)$, which is obtained from the measured normalized gray scale values that represent the oxidized catalyst concentration. The background light intensity $\varphi_{0}$ for natural unperturbed oscillations corresponds to 140 on the gray level scale. The coupling strength, $\mathrm{K}$, is normalized by dividing it by the degree $k_{i}$ of the $i^{\text {th }}$ node, which represents the number of links with other nodes.

The ten oscillators were initially globally coupled to produce in-phase synchronization. The specific topology of node connections was introduced at $t=200.0 \mathrm{~s}$ for $17 \pm 5$ periods. The individual natural periods were measured with constant background intensity and no coupling at the end of the experiment. Experiments were run at various coupling strengths. For data analysis, nodes were re-sorted according to the order of their firing relative to the phase-leader node. This allowed visualization of cluster numbers in the topology based upon their connectivity in the adjacency matrix. The firing sequence of each node was displayed using the recorded gray scale values of all ordered oscillators. These sequences were aligned on top of each other starting with the phase-leader firing pattern during the time of the experiment. The Kuramoto order parameter was used to measure synchronization among all nodes and groups of nodes that form separate clusters. The phase-lag gradient was obtained through linear fitting based on the phase occurrence of each node of each synchronized cluster. 


\subsection{Experimental Results}

\subsubsection{Symmetry Cluster Phase-Lag Synchronization}

The mutual coupling in our 10-node network, consisting of BZ oscillators of broad frequency heterogeneity, results in phase-lag cluster synchronization. At higher values of coupling strength, the oscillator with the highest frequency entrains all other nodes to its frequency. This leads to frequency-locked synchronization among all nodes, which are grouped into a sequence of in-phase clusters. Figure 5.2 summarizes the results of symmetry cluster phase-lag synchronization. Figure 5.2a shows the reduced representation of the original 10-node network in Figure 5.1, where each node or group of nodes that forms a symmetry cluster is represented by one node. The coupling strength and heterogeneity were found to facilitate full entrainment of a sequence of oscillators to the phase leader via the shortest path. Figure 5.2b shows a total of three synchronization groups, which are color based on the network connectivity to the phase leader node (red circle). Figure 5.2c shows the heterogeneity of the system with the natural period of each oscillator, where the mean natural period was $35.04 \mathrm{~s}$ with a standard deviation of $1.04 \mathrm{~s}$. Figure 5.2d shows the firing of each oscillator node, recorded as normalized gray-scale values during the experiment. The results show a wave pattern of phase-lag synchronization through the symmetry clusters, which are color coded as in Figure 5.1. The nodes are renumbered by their connectivity to the phase leader, which are coded as in Figure 5.2b. The order parameter for each cluster was close to 1.0, as shown in Figure 5.2e, indicating in-phase synchronization of the synchronization groups. The global order parameter for all nodes was significantly lower due to the phase lag between synchronization groups. The phase shift between the groups is constant, as demonstrated by a linear fit through the phase of each node 
from each synchronization group, as shown in Figure 5.2f. This result is in contrast to results obtained by Pecora et al. [13], which showed phase-cluster formation according to the underlying permutation symmetries. Our results demonstrate the existence of phase-wave behavior, where there is a constant phase lag between synchronization groups.

a)

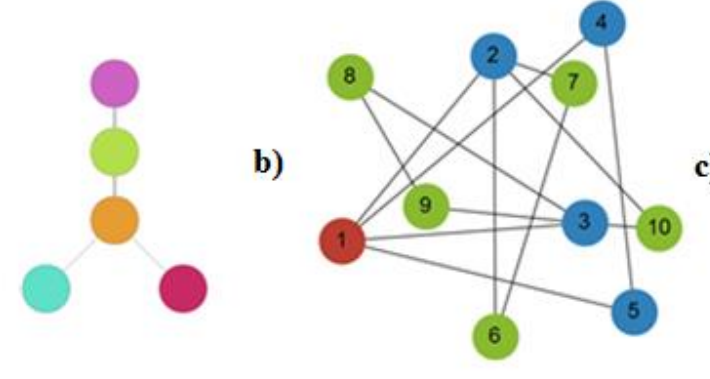

c)

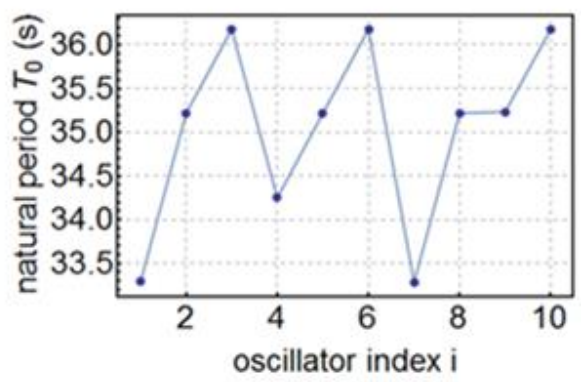

d)

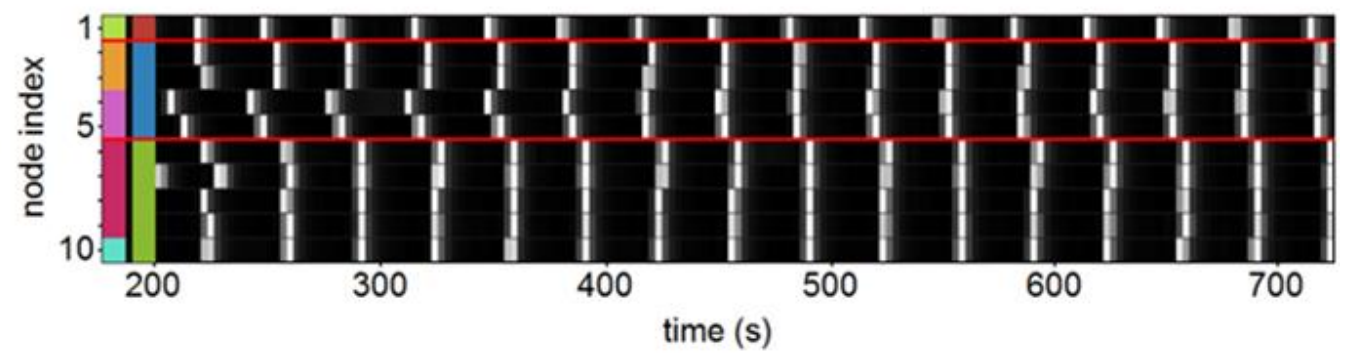

e)

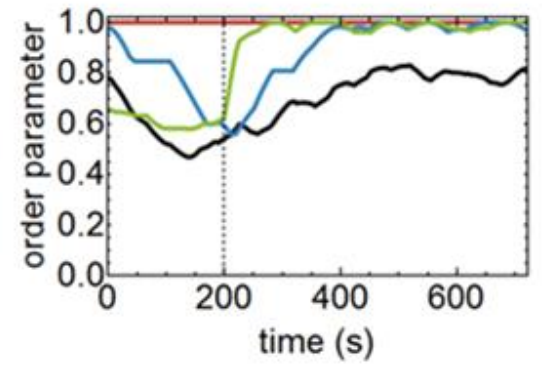

- global

- group 1

- group 2

group 3

f)

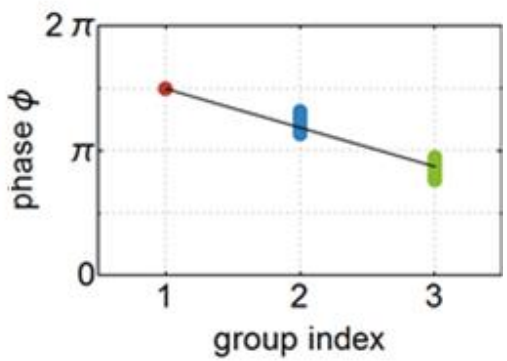

FIG. 5.2: Phase-lag symmetry cluster synchronization. a) A reduced network topology showing the number of symmetry clusters represented by different colors, a simplification of Figure 5.1. b) Network topology showing three synchronization groups, each with simultaneously firing nodes, indicated by different colors (red, blue, and green). The phase leader is node 1 (red), which corresponds to the green node in the symmetry representations of the network in Figure 5.1. c) A plot of the natural period of each oscillator without coupling. d) The recorded gray-scale values for each node ordered by its connectivity to 
the phase leader. The wave pattern of simultaneously firing oscillators is the synchronization groups, which are separated by red lines. The color coding for the symmetry clusters as in Figure 5.1 and for the synchronization groups as in (b). The node index is numbered according to network representation in (b). e) Order parameter after coupling was introduced at $t=200.0 \mathrm{~s}$. The order parameter of all nodes (black plot) shows no overall zero-lag phase synchronization in the system, whereas the order parameter for each synchronization group is close to 1.0, indicating phase synchronization. The color coding is as in (b). f) A linear fit of oscillator phases against their synchronization group index, showing phase-lag synchronization with a constant phase shift; color coding is as in (b). A system of 10 micro-oscillators loaded with $\mathrm{Ru}(\mathrm{bpy})_{3}^{2+}$ catalyst, with a mean natural period of $35.04 \mathrm{~s}$. The coupling strength is $\mathrm{K}=2.0$. The BZ reaction mixture composition: malonic acid, $[\mathrm{MA}]=0.08 \mathrm{M}$; sodium bromide, $[\mathrm{NaBr}]=0.02 \mathrm{M}$; sulfuric acid, $\left[\mathrm{H}_{2} \mathrm{SO}_{4}\right]=0.78 \mathrm{M}$; sodium bromate, $\left[\mathrm{NaBrO}_{3}\right]=0.48 \mathrm{M}$.

\subsubsection{Partial Symmetry Cluster Phase-Lag Synchronization}

In this experiment, the position of the highest frequency oscillator yielded partial symmetry cluster synchronization, with a longer path for the same topology and higher coupling strength, as shown in Figure 5.3a. The role of the highest frequency oscillator to entrain other oscillators was experimentally demonstrated by Kheowan et al. [30], showing that the highest frequency oscillatory source, the pacemaker, entrained the entire BZ medium. However, if entrainment does not lead to complete frequency and phase synchronization, then phase-lag behavior may result [31]. The heterogeneity of the system can be seen from the natural periods of the oscillators, as shown in Figure 5.3b. The mean natural period and standard deviation in the system are $50.65 \mathrm{~s}$ and $3.65 \mathrm{~s}$, respectively. The system resulted in a phase-lag synchronization, which was observed from the gray-scale values of firing events for each oscillator ordered relative to the highest frequency oscillator, as shown in Figure 5.3c. In Figure 5.3d, the order 
parameters of each synchronization group is close to 1.0, which demonstrates synchronization among each group, while the global order parameter of all nodes shows there is no overall zerolag phase synchronization. The phase-lag synchronization is constant with the linear relationship between the phases of each synchronization group, as shown in Figure 5.3e.

a)

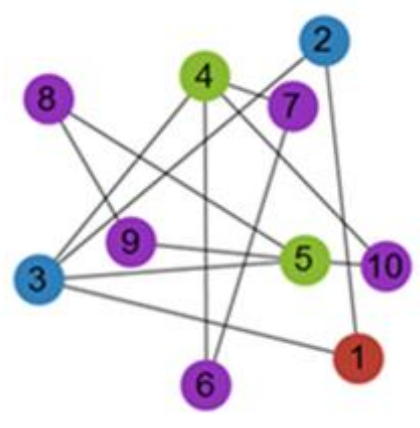

b)

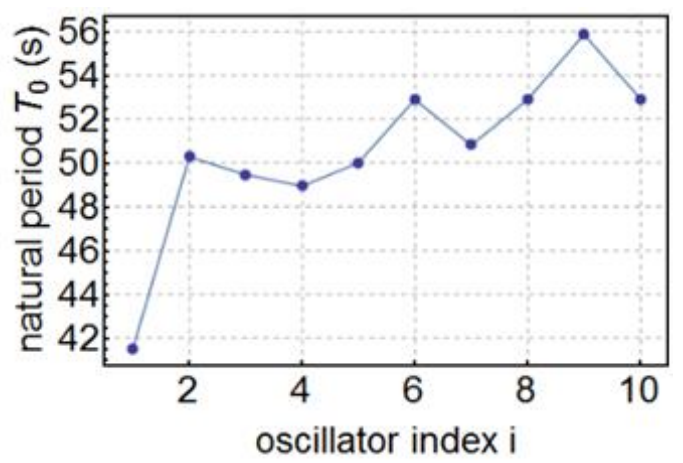

c)

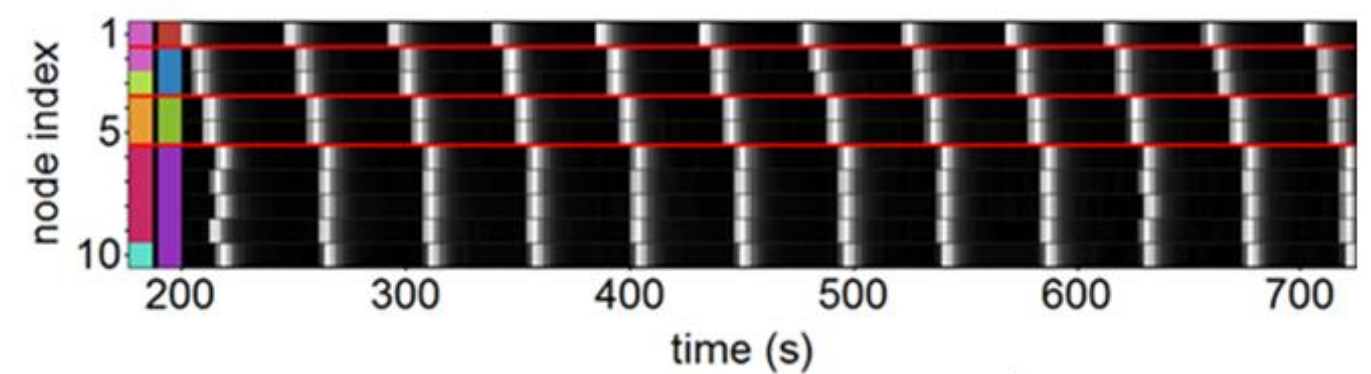

d)

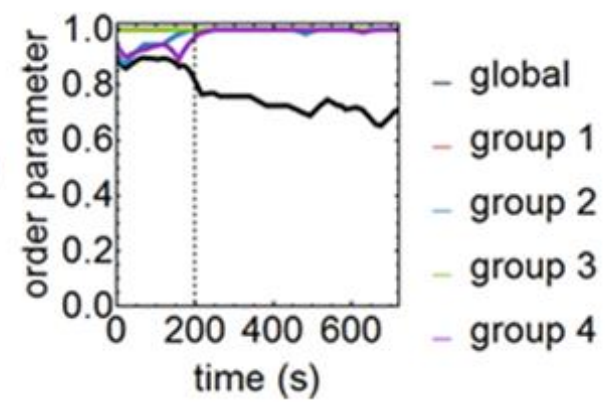

e)

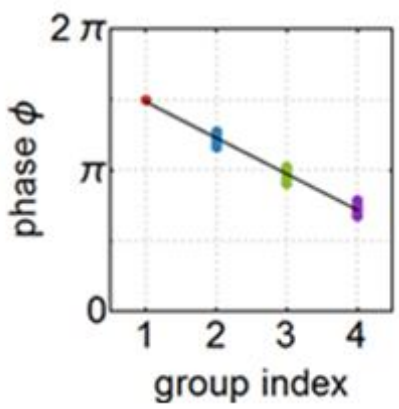

FIG. 5.3: Phase-lag synchronization. a) The network topology showing four groups of simultaneous firing nodes, indicated by different colors (red, blue, green, and magenta). b) A plot of the natural periods of all oscillators with no coupling. c) The recorded gray-scale values for each node, as ordered by connectivity to the phase leader, showing a wave pattern of the synchronization groups, separated by red lines. The phase leader is node 1 (red), which corresponds to one of the nodes forming a symmetry cluster, but not a complete cluster. The color coding for the symmetry clusters is as in Figure 5.1. The node index is 
numbered and colored according to the synchronization groups in (a). d) Order parameter after the coupling introduced at $\mathrm{t}=200.0 \mathrm{~s}$ indicating in-phase synchronization groups, where the order parameter is close to 1.0 for each group. The global order parameter of all nodes (black plot) shows no overall zerolag phase synchronization in this system. The color code is as in (a). e) A linear fit of oscillator phases against the synchronization group index, showing phase-lag synchronization with a constant phase shift; color coding is as in (a). A system of 10 micro-oscillators loaded with a $\mathrm{Ru}(\mathrm{bpy})_{3}^{2+}$ catalyst, with a mean natural period of $50.65 \mathrm{~s}$. The coupling strength is $\mathrm{K}=2.0$. The $\mathrm{BZ}$ reaction mixture composition is the same as in Figure 5.2.

\subsubsection{Partial Synchronization}

Upon decreasing the coupling strength, partial frequency synchronization of two separated groups is observed. Figure 5.4 shows results from an experiment in which coupling strength was not adequate for complete frequency synchronization. Instead, partial synchronization with two groups of different synchronized frequencies was observed, as shown in Figure 5.4. The mean natural period and standard deviation in the system are $55.68 \mathrm{~s}$ and $8.14 \mathrm{~s}$, respectively. After switching to network coupling, the system splits into two groups with different mean synchronized periods ( $57 \mathrm{~s}$ and $52 \mathrm{~s}$ ), as shown in Figure 5.4b. In this experiment, the phase leader node entrained most of the nodes, except for nodes 8 and 9, which appear in the bounded blue lines in Figure 5.4c. This leads to partial phase-lag synchronization within the separated group, as seen from the deviation of the wave-form pattern from complete phase-lag synchronization based on network connectivity. Partial synchronization was predicted by Winfree [32] in his model of coupled identical oscillators, where increasing coupling strength allows for cluster synchrony after incoherent behavior. However, his model suggested phase coherence with complete synchronization in phase and frequency, while our non-identical 
oscillators showed frequency and phase-lag synchronization with increased coupling strength, as shown previously in Figures 5.2 and 5.3.

a)

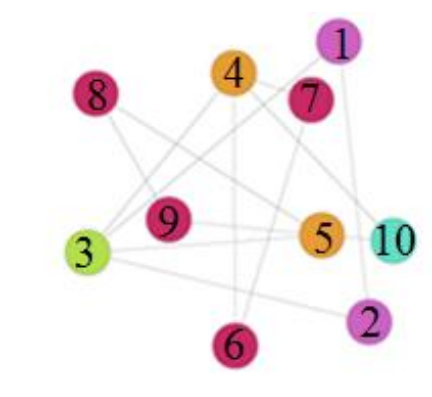

b)

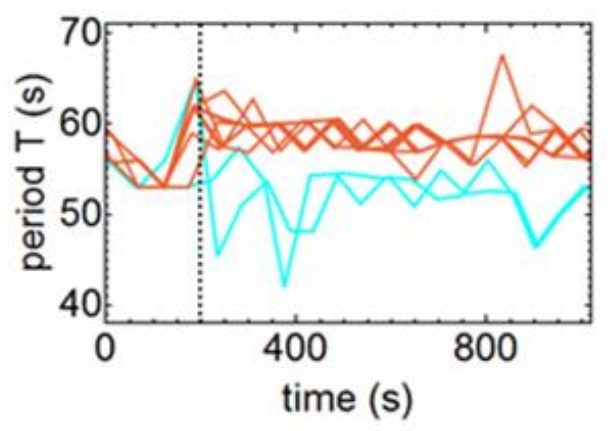

c)

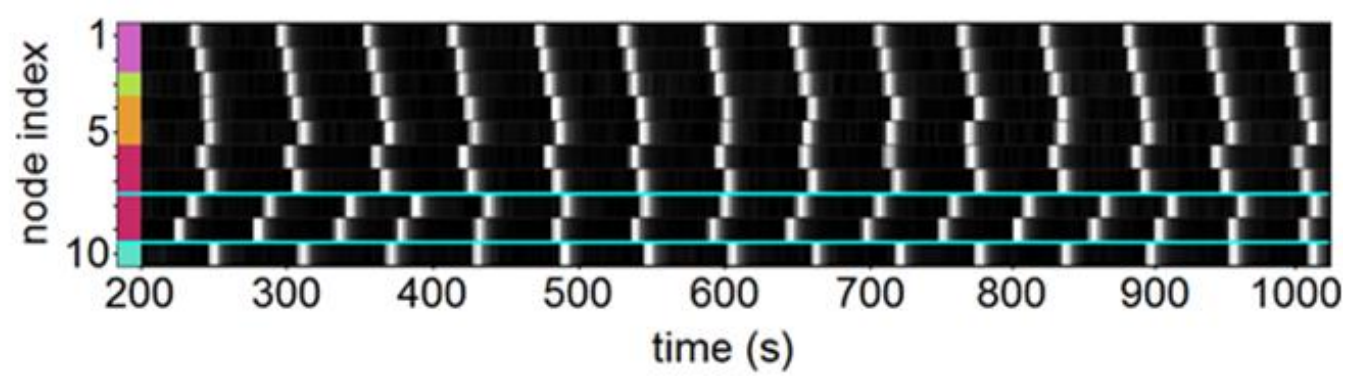

FIG. 5.4: Partial frequency synchronization. a) The 10-node network topology of all permutation symmetry clusters shown with different colors. b) A plot of the periods of all beads after switching on coupling, showing splitting into two synchronized periods, which indicates partial frequency synchronization. c) The recorded gray-scale values for each node, as ordered by connectivity to the phase leader. Deviation of phase-lag patterns in nodes 8 and 9 is observed. The node index is numbered and colored according to network representation in (a). A system of 10 micro-oscillators loaded with $\mathrm{Ru}(\mathrm{bpy})_{3}^{2+}$ catalyst, with a mean natural period of $55.68 \mathrm{~s}$. The coupling strength is $\mathrm{K}=0.60$. The BZ reaction mixture composition is the same as in Figure 5.2.

\subsubsection{Independent Phase Oscillations}

Upon further decreasing the coupling strength, no form of synchronization was obtained in the network. The oscillator of highest frequency could not entrain any of the symmetry clusters 
shown in Figure 5.5a. Figure 5.5b shows a wide distribution of periods after coupling and independent oscillations during the experiment. The mean natural period and the standard deviation with no coupling were $55.97 \mathrm{~s}$ and $1.95 \mathrm{~s}$, respectively. There was no phase coherence, as indicated by the pattern of firing of each node according to gray-scale recordings, as shown in Figure 5.5c.

a)

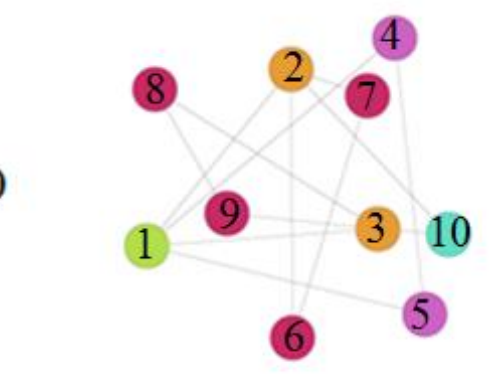

b)

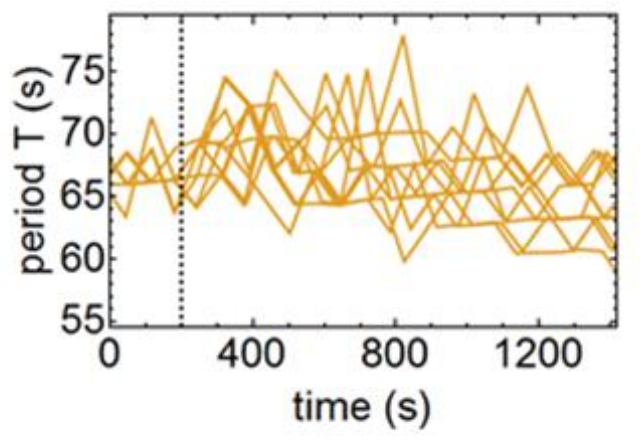

c)

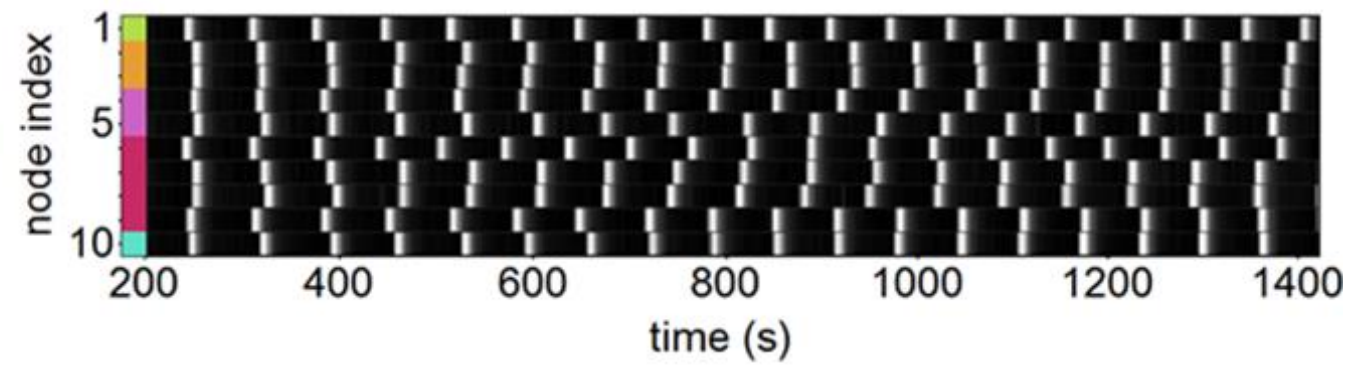

FIG. 5.5: Incoherent oscillation. a) The 10-node network topology of all permutation symmetry clusters with different colors. b) A plot of the periods of all beads after switching on coupling. A wide distribution of periods indicates no synchronization. c) The recorded gray-scale values for each node, showing an irregular pattern. The node index is numbered and colored according to network representation in (a). A system of 10 micro-oscillators loaded with $\mathrm{Ru}(\mathrm{bpy})_{3}^{2+}$ catalyst, with a mean natural period of $55.97 \mathrm{~s}$. The coupling strength is $\mathrm{K}=0.05$. The $\mathrm{BZ}$ reaction mixture composition is the same as in Figure 5.2. 


\subsection{Discussion}

We considered a network of 10 non-identical, photosensitive, BZ micro-oscillators. The network connection is bidirectional between node pairs and is represented mathematically using an adjacency matrix. Photochemical feedback is used to vary the coupling strength and different synchronization behaviors are observed. We chose a network with an underlying permutation symmetry to investigate synchronization. In our study, we observed a wave pattern of symmetry cluster synchronization. Phase-lag propagation is initiated by a phase leader, with the phase leader resembling a pacemaker as the wave source [30]. The phase leader represents the node of the highest natural frequency in the network. However, unlike entrainment by a pacemaker in a continuous medium, where oscillators undergo diffusive coupling, the entrainment of oscillators by a phase leader in a network depends on the hierarchical connectivity of links in the topology.

The heterogeneity in our system did not allow zero-lag synchronization. Instead, we obtain phase-lag synchronization when an appropriate coupling strength threshold is satisfied. The phase leader influences the nearest oscillators, which form a phase-locked synchronization group, and then the oscillators further away are similarly synchronized. As was theoretically shown, the ability for a system to synchronize depends on both coupling strength and frequency heterogeneity, with the coupling strength needing to be above a critical value for frequency synchronization [33]. The clusters that are entrained by the phase leader form frequency-locked synchronization groups in a discrete heterogeneous medium. Similar phase-lag behavior was described in the swimming and coherent motion of lamprey fish [15-18].

We focused on synchronization behaviors as a function of coupling strength. Our results are relevant to theoretical results on motor behavior, which show that the type of phase-lag relationships observed in locomotion is dependent on coupling strength and/or frequency 
heterogeneity of the oscillators $[18,20,34]$. When applying higher coupling strength, we observed phase-lag synchronization, where the system splits into synchronization groups that are shifted in time with respect to each other. This can be considered to be a case of group synchrony with phase-lag synchronization, which was first suggested by Rosenblum et al. [14] in their theoretical work on mutually coupled, non-identical chaotic oscillators. Lag synchronization has also been observed experimentally in circuits of electronic oscillators [35, 36] and lasers [37]. In our system, there is frequency locking among all oscillators in the network. In addition to frequency locking, each synchronization group is locked to the same phase. Moreover, the synchronized behavior that we observed exhibited phase-lag behavior. Phase clusters in a network with no phase-lag were demonstrated theoretically in a network of coupled identical chaotic oscillators $[38,39]$. In addition, our results differ from the recent experimental results obtained by Pecora et al. [13], who found phase clusters instead of phase-lag synchronization groups with coupled identical optical oscillators. Our observations correlate with the suggested phase-lag synchronization mechanisms for some brain functions, such as locomotion $[15,16]$, attention [40], and memory [41].

The Pecora et al. experiments [13] reveal how the inherent symmetry in networks can lead to symmetry cluster synchronization. In our study, when coupling is adequate, the position of the highest frequency oscillator determines whether one obtains complete or partial symmetry cluster phase-lag synchronization. Different pathways of entrainment are observed according to the phase-leader placement. Phase-lag synchronization follows the shortest path if symmetry cluster synchronization is obtained. This is important, because a network's underlying symmetry can provide the shortest path and, thus, allow network robustness and simplification without losing the information. This was supported theoretically in food web networks [42] and 
in protein network interactions [43], where some nodes possess more symmetry than others, which allows for reduction of an entire network.

The position of the highest frequency oscillator may cause phase-lag synchronization via partial symmetry clusters with longer paths for the same topology. Different paths with different clusters confirm the biological view of synchronization. For example, in information coding, neurons re-participate over and over in different cluster formations to facilitate different contexts rather than having specialized neurons assembled for each specific action [44-46]. This has been demonstrated in changes in neural patterns in visual, parietal, and motor areas based on different contexts of cat activity [47]. In addition, functional heterogeneity of cells is observed experimentally among segmental oscillators that produce a leech swimming rhythm with a longer path [48].

This study has demonstrated that frequency synchronization coexists with phasesynchronized groups. By increasing the coupling strength, there is a transition from non-coherent oscillations to partially synchronized oscillations, and eventually to coherent phase-lag synchronization groups. A model of coupled neural networks shows parallel phase-cluster synchronization, which can lead to zero phase-lag synchronization, with or without delay [49, 50]. In our system, however, we attained global frequency synchronization among a sequence of phase-lag synchronization groups, each with zero-lag synchronization, by increasing coupling strength.

\subsection{Summary}

Networks are important and widespread in the real world, such as in social, biological, and manmade networks. Many theoretical studies have been performed to understand the dynamics of networks and their architectures of interacting ensembles [51-54]. Some studies have been 
empirical [12, 55-59], and some focused on network symmetry while studying phase-locked relations [13, 60, 61]. Many theoretical [38, 39] and empirical [5-13] studies that focused on phase synchronization have revealed the phenomenon of phase-cluster synchronization. In contrast, we demonstrated the existence of phase-lag synchronization groups in coupled, nonidentical BZ oscillators with bidirectional network connections. This study used a small network with underlying symmetry to mimic an actual network system, such as a protein network [43], food web network [42], or cortices network [62, 63]. Different behaviors are observed while varying the coupling strength in the system. By introducing topology coupling, all nodes of the same frequency were organized into groups of phase-synchronized oscillators at appropriate coupling strengths. The combination of synchronization groups and phase-lag synchronization is a new type of synchronization behavior. It represents groups of frequency-synchronized oscillators with a constant phase difference among consecutive groups based on their network connectivity. In our heterogeneous system, the transition of a non-phase coherent state to phaselag synchronization groups is obtained with increased coupling strength. This is consistent with the theoretical demonstration of adding delays in assemblies of excitatory connections, which can prevent zero-lag synchronization in neural model representations [64, 65]. In addition, these results shed light on the mechanism of phase-lag synchronization in some brain functions [15, 16, 40, 41]. Accordingly, this study mimics the behavior of real biological networks involving clustering with a non-zero phase-lag mechanism.

Our work shows that networks with an underlying symmetry have different pathways of synchronization depending on circumstances. Some theoretical studies have investigated the controllability of networks to recover a lost network function [22] and to control network robustness and signal transmission [66]. Here, we suggest the need for further study on 
controlling network dynamics by imposing different pathways in the same topology based on knowledge of network symmetry. Future results could provide insights into the mechanisms involved in the control of real-world networks. This can be achieved by stimulation of one or more nodes to choose a possible dynamic pathway. Pathways of synchronization may also be controlled through weighted coupling between links. The results may provide a better understanding of the dynamics of neural network modulations [67]. 


\section{Reference}

[1] W. Singer, "Synchronization of cortical activity and its putative role in information processing and learning.” Annu. Rev. Physiol. 55, 349-374 (1993).

Online Version

[2] C. D. Gilbert and J. P. Kelly, "The projections of cells in different layers of the cat's visual cortex.” J. Comp. Neurol. 163, 81-105 (1975).

Online Version

[3] C. D. Gilbert and T. N. Wiesel, "Clustered intrinsic connections in cat visual cortex." J. Neurosci. 3, 1116-1133 (1983).

Online Version

[4] T. J. Imig and J. F. Brugge, "Sources and terminations of callosal axons related to binaural and frequency maps in primary auditory cortex of the cat." J. Comp. Neurol. 182, 637-660 (1978).

Online Version

[5] P. S. Goldman and W. J. H. Nauta, "Columnar distribution of cortico-cortical fibers in the frontal association, limbic, and motor cortex of the developing rhesus monkey." Brain Res. 122, 393-413 (1977).

Online Version

[6] D. J. Pedrosa, C. Reck, E. Florin, K. A. M. Pauls, M. Maarouf, L. Wojtecki, H. S. Dafsari, V. Sturm, A. Schnitzler, G. R. Fink, and L. Timmermann, "Essential tremor and tremor in Parkinson's disease are associated with distinct "tremor clusters" in the ventral thalamus." Exp. Neurol. 237, 435-443 (2012).

Online Version 
[7] A. F. Taylor, M. R. Tinsley, F. Wang, and K. Showalter, "Phase clusters in large populations of chemical oscillators.” Angew. Chem. Int. Ed. Engl. 50, 10161-10164 (2011).

Online Version

[8] A. F. Taylor, P. Kapetanopoulos, B. J. Whitaker, R. Toth, L. Bull, and M. R. Tinsley, "Clusters and switchers in globally coupled photochemical oscillators." Phys. Rev. Lett. 100, 214101 (2008).

Online Version

[9] I. Z. Kiss, Y. Zhai, and J. L. Hudson, "Predicting mutual entrainment of oscillators with experiment-based phase models.” Phys. Rev. Lett. 94, 248301 (2005).

Online Version

[10] H. Varela, C. Beta, A. Bonnefont, and K. Krischer, "A hierarchy of global coupling induced cluster patterns during the oscillatory $\mathrm{H}_{2}$-electrooxidation reaction on a Pt ringelectrode.” Phys. Chem. Chem. Phys. 7, 2429-2439 (2005).

Online Version

[11] I. Z. Kiss, C. G. Rusin, H. Kori, and J. L. Hudson, "Engineering complex dynamical structures: Sequential patterns and desynchronization." Science 316, 1886-1889 (2007).

Online Version

[12] C. R. S. Williams, F. Sorrentino, T. E. Murphy, and R. Roy, "Synchronization states and multistability in a ring of periodic oscillators: Experimentally variable coupling delays." Chaos 23, 043117 (2013).

Online Version 
[13] L. M. Pecora, F. Sorrentino, A. M. Hagerstrom, T. E. Murphy, and R. Roy, "Cluster synchronization and isolated desynchronization in complex networks with symmetries." Nat. Commun. 5, 4079 (2014).

Online Version

[14] M. G. Rosenblum, A. S. Pikovsky, and J. Kurths, "From phase to lag synchronization in coupled chaotic oscillators.” Phys. Rev. Lett. 78, 4193-4196 (1997).

Online Version

[15] J. H. Kotaleski, A. Lansner, and S. Grillner, "Neural mechanisms potentially contributing to the intersegmental phase lag in lamprey." Biol. Cybern. 81 299-315 (1999).

Online Version

[16] A. H. Cohen, G. B. Ermentrout, T. Kiemel, N. Kopell, K. A. Sigvardt, and T. L. Williams, "Modelling of intersegmental coordination in the lamprey central pattern generator for locomotion." Trends Neurosci. 15, 434-438 (1992).

Online Version

[17] F. K. Skinner and B. Mulloney, "Intersegmental coordination in invertebrates and vertebrates.” Curr. Opin. Neurobiol. 8, 725-732 (1998).

Online Version

[18] A. A. V. Hill, M. A. Masino, and R. L. Calabrese, "Intersegmental coordination of rhythmic motor patterns.” J. Neurophysiol. 90, 531-538 (2003).

Online Version

[19] F. J. Eisenhart, T. W. Cacciatore, and W. B. Kristan, "A central pattern generator underlies crawling in the medicinal leech.” J. Comp. Physiol. A. 186, 631-643 (2000). 
Online Version

[20] M. Ullström, J. H. Kotaleski, J. Tegnér, E. Aurell, S. Grillner, and A. Lansner, "Activity-dependent modulation of adaptation produces a constant burst proportion in a model of the lamprey spinal locomotor generator.” Biol. Cybern.79, 1-14 (1998).

Online Version

[21] T. W. Cacciatore, R. Rozenshteyn, and W. B. Kristan, "Kinematics and modeling of leech crawling: Evidence for an oscillatory behavior produced by propagating waves of excitation.” J. Neurosci. 20, 1643-1655 (2000).

Online Version

[22] E. Marder and D. Bucher, "Central pattern generators and the control of rhythmic movements." Curr. Biol. 11, R986-R996 (2001).

Online Version

[23] P. Ortoleva and J. Ross, "Phase waves in oscillatory chemical reactions." J. Chem. Phys. 58, 5673-5680 (1973).

Online Version

[24] J. M. Bodet, J. Ross, and C. Vidal, "Experiments on phase diffusion waves." J. Chem. Phys. 86, 4418-4424 (1987).

Online Version

[25] R. R. Aliev, "Oscillation phase dynamics in the Belousov-Zhabotinsky reaction. Implementation to image processing.” J. Phys. Chem. 98, 3999-4002 (1994).

Online Version 
[26] V. K. Vanag, L. Yang, M. Dolnik, A. M. Zhabotinsky, and I. R Epstein, “Oscillatory cluster patterns in a homogeneous chemical system with global feedback." Nature 406, 389-391 (2000).

Online Version

[27] S. R. Jones, B. Mulloney, T. J. Kaper, and N. Kopell, "Coordination of cellular patterngenerating circuits that control limb movements: The sources of stable differences in intersegmental phases.” J. Neurosci. 23, 3457-3468 (2003).

Online Version

[28] D. Garlaschelli, F. Ruzzenenti, and R. Basosi, "Complex networks and symmetry I: A review." Symmetry 2, 1683-1709 (2010).

Online Version

[29] B. D. MacArthur, R. J. Sánchez-García, and J. W. Anderson, "Symmetry in complex networks.” Discrete Appl. Math. 156, 3525-3531 (2008).

Online Version

[30] O.-U. Kheowan, E. Mihaliuk, B. Blasius, I. Sendiña-Nadal, and K. Showalter, "Wave mediated synchronization of nonuniform oscillatory media.” Phys. Rev. Lett. 98, 074101 (2007).

Online Version

[31] A. Pikovsky, M. Rosenblum, and J. Kurths, Synchronization: A universal concept in nonlinear sciences (Cambridge University Press, Cambridge, 2003).

Online Version

[32] A. T. Winfree, "Biological rhythms and the behavior of populations of coupled oscillators.” J. Theor. Biol. 16, 15-42 (1967). 
Online Version

[33] P. C. Matthews and S. H. Strogatz, "Phase diagram for the collective behavior of limitcycle oscillators.” Phys. Rev. Lett. 65 1701-1704 (1990).

Online Version

[34] N. Kopell and G. B. Ermentrout, "Symmetry and phaselocking in chains of weakly coupled oscillators.” Comm. Pure Appl. Math. 39, 623-660 (1986).

Online Version

[35] S. Taherion, and Y.-C. Lai, “Observability of lag synchronization of coupled chaotic oscillators.” Phys. Rev. E. 59, R6247-R6250 (1999).

Online Version

[36] L. Zhu and Y.-C. Lai, "Experimental observation of generalized time-lagged chaotic synchronization.” Phys. Rev. E. 64, 045205 (2001).

Online Version

[37] T. Heil, I. Fischer, W. Elsässer, J. Mulet, and C. R. Mirasso, "Chaos synchronization and spontaneous symmetry-breaking in symmetrically delay-coupled semiconductor lasers.” Phys. Rev. Lett. 86, 795-798 (2001)

Online Version

[38] V. N. Belykh, I. V. Belykh, and E. Mosekilde, "Cluster synchronization modes in an ensemble of coupled chaotic oscillators.” Phys. Rev. E. 63, 036216 (2001).

Online Version

[39] V. N. Belykh, G. V. Osipov, V. S. Petrov, J. A. K. Suykens, and J. Vandewalle, “Cluster synchronization in oscillatory networks.” Chaos 18, 037106 (2008).

Online Version 
[40] G. G. Gregoriou, S. J. Gotts, H. Zhou, and R. Desimone, "High-Frequency, long-range coupling between prefrontal and visual cortex during attention.” Science 324, 12071210 (2009).

Online Version

[41] K. Benchenane, A. Peyrache, M. Khamassi, P. L. Tierney, Y. Gioanni, F. P. Battaglia, and S. L. Wiener, "Coherent theta oscillations and reorganization of spike timing in the hippocampal-prefrontal network upon learning." Neuron 66, 921-936 (2010).

Online Version

[42] H. Aufderheide, L. Rudolf, and T. Gross, "Mesoscale symmetries explain dynamical equivalence of food webs.” New J. Phys. 14, 105014 (2012).

Online Version

[43] H. Jeong, S. P. Mason, A.-L. Barabási, and Z. N. Oltvai, "Lethality and centrality in protein networks." Nature 411, 41-42 (2001).

Online Version

[44] W. Singer, "The formation of cooperative cell assemblies in the visual cortex." J. Exp. Biol. 153, 177-197 (1990).

Online Version

[45] E. M. Callaway and L. C. Katz, "Emergence and refinement of clustered horizontal connections in cat striate cortex." J. Neurosci. 10, 1134-1153 (1990).

Online Version

[46] A. Riehle, S. Grün, M. Diesmann, and A. Aertsen, "Spike synchronization and rate modulation differentially involved in motor cortical function." Science 278, 1950-1953 (1997). 
Online Version

[47] P. R. Roelfsema, A. K. Engel, P. König, and W. Singer, "Visuomotor integration is associated with zero time-lag synchronization among cortical areas.” Nature 385, 157161 (1997).

Online Version

[48] C. G. Hocker, X. Yu, and W. O. Friesen, "Functionally heterogeneous segmental oscillators generate swimming in the medical leech.” J. Comp. Physiol. A. 186, 871$883(2000)$

Online Version

[49] D. Wang, "Emergent synchrony in locally coupled neural oscillators." IEEE Trans. on Neural Netw. 6, 941-948 (1995).

Online Version

[50] P. König and T. B. Schillen, "Stimulus-dependent assembly formation of oscillatory responses: I. Synchronization.” Neural Comput. 3, 155-166 (1991).

Online Version

[51] O. Sporns, G. Tononi, and G. M. Edelman, "Theoretical neuroanatomy: Relating anatomical and functional connectivity in graphs and cortical connection matrices." Cereb. Cortex 10, 127-141 (2000).

Online Version

[52] S. H. Strogatz, "Exploring complex networks.” Nature 410, 268-276 (2001). Online Version

[53] R. Albert and A.-L. Barabási, "Statistical mechanics of complex networks.” Rev. Mod. Phys. 74, 47-97 (2002). 
Online Version

[54] A. Arenas, A. Díaz-Guilera, J. Kurths, Y. Moreno, and C. Zhou, "Synchronization in complex networks.” Phys. Rep. 469, 93-153 (2008).

Online Version

[55] H. Jeong, B. Tombor, R. Albert, Z. N. Oltvai, and A.-L. Barabási, "The large-scale organization of metabolic networks.” Nature 407, 651-654 (2000).

Online Version

[56] B. Ravoori, A. B. Cohen, J. Sun, A. E. Motter, T. E. Murphy, and R. Roy, "Robustness of optimal synchronization in real networks.” Phys. Rev. Lett. 107, 034102 (2011).

Online Version

[57] A. M. Hagerstrom, T. E. Murphy, R. Roy, P. Hövel, I. Omelchenko, and E. Schöll, "Experimental observation of chimeras in coupled-map lattices." Nature Phys. 8, 658$661(2012)$.

Online Version

[58] M. Wickramasinghe and I. Z. Kiss, "Spatially organized partial synchronization through the chimera mechanism in a network of electrochemical reactions." Phys. Chem. Chem. Phys. 16, 18360-18369 (2014).

Online Version

[59] M. Oka, H. Masuda, Y. Kaneda, and S. Kubota, "Laser-diode-pumped phase-locked Nd:YAG laser arrays.” IEEE J. Quant. Electron. 28, 1142-1147 (1992).

Online Version

[60] J. J. Collins and I. N. Stewart, "Coupled nonlinear oscillators and the symmetries of animal gaits.” J. Nonlinear Sci. 3, 349-392 (1993). 
Online Version

[61] M. Wickramasinghe and I. Z. Kiss, "Spatially organized dynamical states in chemical oscillator networks: Synchronization, dynamical differentiation, and chimera patterns.” PLoS ONE 8, e80586 (2013).

Online Version

[62] C. M. Gray, "The temporal correlation hypothesis of visual feature integration: Still alive and well." Neuron 24, 31-47 (1999).

Online Version

[63] F. Varela, J.-P. Lachaux, E. Rodriguez, and J. Martinerie, "The brainweb: Phase synchronization and large-scale integration.” Nat. Rev. Neurosci. 2, 229-239 (2001).

Online Version

[64] M. Zeitler, A. Daffertshofer, and C. C. Gielen, "Asymmetry in pulse-coupled oscillators with delay.” Phys. Rev. E. 79, 065203 (2009).

Online Version

[65] U. Ernst, K. Pawelzik, and T. Geisel, "Synchronization induced by temporal delays in pulse-coupled oscillators.” Phys. Rev. Lett. 74, 1570-1573 (1995).

Online Version

[66] J. C. Nacher and T. Akutsu, "Dominating scale-free networks with variable scaling exponent: Heterogeneous networks are not difficult to control." New J. Phys. 14, 073005 (2012)

Online Version 
[67] R. M. Harris-Warrick and E. Marder, "Modulation of neural networks for behavior." Annu. Rev. Neurosci. 14, 39-57 (1991).

Online Version 
Synchronization of Coupled and Periodically Forced Chemical Oscillators

Razan Snari

Dissertation submitted to the Eberly College of Arts and Sciences at West Virginia University in partial fulfillment of the requirements for the degree of

Doctor of Philosophy in Chemistry

C. Eugene Bennett Department of Chemistry

APPROVAL OF THE EXAMINING COMMITTEE

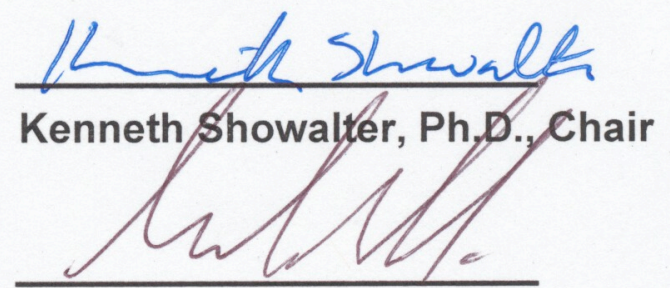

Charles Jaffé, Ph.D.

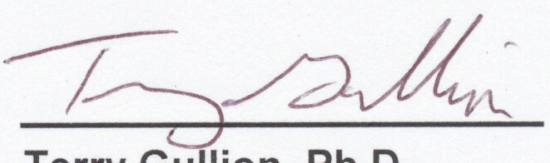

Terry Gullion, Ph.D.

$\frac{M \operatorname{mog} 27,2015}{\text { Date }}$
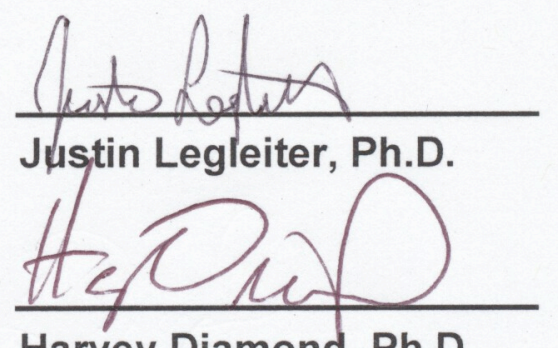

Harvey Diamond, Ph.D.
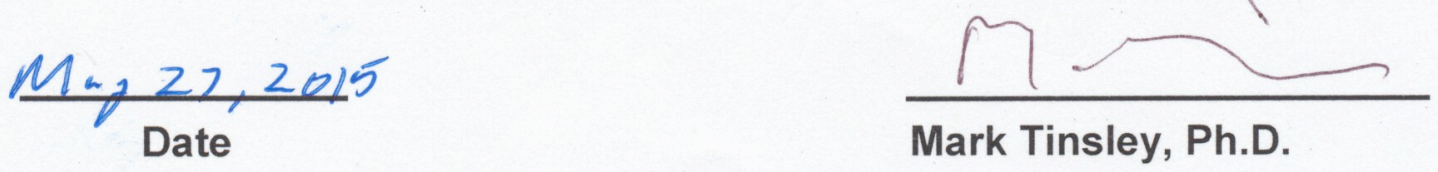

Mark Tinsley, Ph.D. 